\title{
Analytic solutions for a Stefan problem with Gibbs-Thomson correction
}

By Joachim Escher at Hannover, Jan Prüss at Halle, and Gieri Simonett at Nashville

\begin{abstract}
We provide existence of a unique smooth solution for a class of one- and two-phase Stefan problems with Gibbs-Thomson correction in arbitrary space dimensions. In addition, it is shown that the moving interface depends analytically on the temporal and spatial variables. Of crucial importance for the analysis is the property of maximal $L_{p^{-}}$ regularity for the linearized problem, which is fully developed in this paper as well.
\end{abstract}

\section{Introduction and main results}

The Stefan problem is a model for phase transitions in liquid-solid systems and accounts for heat diffusion and exchange of latent heat in a homogeneous medium. The strong formulation of this model corresponds to a free boundary problem involving a parabolic diffusion equation for each phase and a transmission condition prescribed at the interface separating the phases.

In order to describe the physical situation in some more detail, let us consider a domain $\Omega$ that is occupied by a liquid and a solid phase, say water and ice, that are separated by an interface $\Gamma$. Due to melting or freezing, the corresponding regions occupied by water and ice will change and, consequently, the interface $\Gamma$ will also change its position and shape. This leads to a free boundary problem.

The basic physical laws governing this process are conservation of mass and conservation of energy. The unknowns are the temperature $u^{+}$and $u^{-}$for the liquid and solid phase, respectively, and the position of the interface $\Gamma$ separating the two different phases. The conservation laws can then be expressed by a diffusion equation for $u^{+}$and $u^{-}$in the respective regions $\Omega^{+}$and $\Omega^{-}$occupied by the liquid and solid phase and by the socalled Stefan condition which accounts for the exchange of latent heat due to melting or solidifying. In the classical Stefan problem one assumes that the temperatures $u^{+}$and $u^{-}$

The research of the third author has been partially supported by the Vanderbilt University Research Council and by NSF Grant DMS-9801337. 
coincide at the interface $\Gamma$ (where the two phases are in contact), that is, one requires

$$
u^{+}=u^{-}=0 \quad \text { on } \Gamma
$$

where 0 is the melting temperature. Molecular considerations attempting to explain the effect of supercooling and of dendritic growth of crystals suggest that the condition (1.1) on the free boundary $\Gamma$ be replaced by the Gibbs-Thomson correction

$$
u^{+}=u^{-}=\sigma \kappa \quad \text { on } \Gamma
$$

where $\sigma$ is a positive constant, called the surface tension, and where $\kappa$ denotes the mean curvature of $\Gamma$. We will occasionally refer to the Stefan problem with condition (1.2) as the Stefan problem with surface tension.

It should be emphasized that the Stefan problem with Gibbs-Thomson correction (1.2) differs from the classical Stefan problem in a much more fundamental way than just in the modification of an interface condition. This becomes evident, for instance, by the fact that the classical Stefan problem allows for a comparison principle, a property that is no longer shared by the Stefan problem with surface tension. As a consequence, many of the techniques that were developed and successfully applied to the classical Stefan problem cannot be used for the modified problem. A striking difference is also provided by the fact that in the classical Stefan model, the temperature completely determines the phases, that is, the liquid region can be characterized by the condition $u>0$, whereas $u<0$ characterizes the solid region, with $u=0$ being the melting temperature. The inclusion of surface tension will no longer allow to determine the phases merely by the sign of $u$.

The main reason for introducing the Gibbs-Thomson correction (1.2) stems from the need to account for so-called supercooling, in which a fluid supports temperatures below its freezing point, or superheating, the analogous phenomena for solids; or dendrite formation, in which simple shapes evolve into complicated tree-like structures. The effect of supercooling can be in the order of hundreds of degrees for certain materials and is required for nucleation, namely the forming of a new phase in a set previously occupied by the parental phase, see [12], Chapter 1, and [52]. We also refer to [11], [12], [23], [27], [28], [30], [35], [52] for more information.

The Stefan problem has been studied in the mathematical literature for over a century, see [47], [39] and [53], pp. 117-120, for a historic account, and has attracted the attention of many prominent mathematicians.

The classical Stefan problem is known to admit unique global weak solutions, see for instance [20], [21], [31] and [34], pp. 496-503. It is important to point out that the existence of weak solutions is closely tied to the maximum principle, see for instance the proofs in $[20]$.

Important results concerning the regularity of weak solutions for the multidimensional classical one-phase Stefan problem were established in [7], [8], [10], [22], [32], [33], [37], and regularity results for the classical two-phase Stefan problem are contained in [4], [5], [9], [14], [15], [41], [48], [55], to mention only a few references. We mention that classical solutions for the Stefan problem with condition (1.1) are established in [29], [38]. 
Although the Stefan problem with the Gibbs-Thomson correction (1.2) has been around for many decades, only few analytical results concerning existence and the regularity of solutions are known, see [23], [36], [40], [45], [46]. The authors in [23] consider the case with small surface tension $0<\sigma \ll 1$ and linearize the problem about $\sigma=0$. Assuming the existence of smooth solutions for the case $\sigma=0$, that is, for the classical Stefan problem, the authors prove existence and uniqueness of a weak solution for the linearized problem and then investigate the effect of small surface tension on the shape of $\Gamma(t)$. Existence of global weak solutions is established in [36], using a discretized problem and a capacitytype estimate for approximating solutions. The weak solutions obtained in [36] have a sharp interface, but are highly non-unique. In [40], the way in which a spherical ball of ice in a supercooled fluid might melt down is investigated. A proof for existence (without uniqueness) of classical solutions was given in [45], [46].

If the diffusion equation $\partial_{t} u^{ \pm}-\Delta u^{ \pm}=0$ in $\Omega^{ \pm}$is replaced by the elliptic equation $\Delta u^{ \pm}=0$, then the resulting problem is the quasi-stationary Stefan problem with surface tension, which has also been termed the Mullins-Sekerka model (or the Hele-Shaw model with surface tension). Existence, uniqueness, and regularity of classical solutions for the quasi-stationary approximation has recently been investigated in [6], [13], [16], [18], [19].

We should point out that uniqueness of solutions for the Stefan problem with GibbsThomson correction has not been established so far. This provides a serious drawback for a complete understanding of the problem. The lack of uniqueness would for instance imply that the process of melting and freezing is not completely predictable, as the same initial configuration can lead to different outcomes.

In this paper we close this gap. Moreover, we are able to show that solutions regularize, and that the free boundary is even analytic in time and space. In order to obtain our results we devise a new approach based on maximal regularity. The thrust of this approach is manifold. It gives the best possible estimates for the linearized problem. This in turn allows us to use the contraction principle to obtain unique solutions for the nonlinear problem. Moreover, our approach allows us to resort to the implicit function theorem to establish further properties of solutions, such as regularity.

After these general remarks we shall now introduce the precise mathematical model for the Stefan problem with Gibbs-Thomson correction. We will, in fact, look at the special geometry where the free boundary is represented as the graph of a function. The general situation will be treated - among other topics - in a forthcoming paper.

Let us then consider a family $\Gamma=\{\Gamma(t) ; t \geqq 0\}$ of hypersurfaces in $\mathbb{R}^{n+1}$, where each individual hypersurface is assumed to be a graph over $\mathbb{R}^{n}$, that is, $\Gamma(t)=\operatorname{graph}(\rho(t))$ for some $\rho(t) \in C^{2}\left(\mathbb{R}^{n}, \mathbb{R}\right)$. Moreover, let $\Omega^{+}(t)$ and $\Omega^{-}(t)$ denote the domain above and below $\Gamma(t)$, respectively, that is,

$$
\Omega^{ \pm}(t):=\left\{(x, y) \in \mathbb{R}^{n} \times \mathbb{R} ; y \gtrless \rho(t, x)\right\} .
$$

We set $\Omega(t):=\Omega^{+}(t) \cup \Omega^{-}(t)$ and consider the following problem: Given $\Gamma_{0}=\operatorname{graph}\left(\rho_{0}\right)$ and $u_{0}: \Omega(0) \rightarrow \mathbb{R}$, determine a family $\Gamma=\{\Gamma(t) ; t \geqq 0\}$ and a function

$$
u: \bigcup_{t \geqq 0}(\{t\} \times \Omega(t)) \rightarrow \mathbb{R}
$$


such that

$$
\begin{cases}\partial_{t} u-\Delta u=0 & \text { in } \Omega(t), t>0 \\ u=\kappa(t) & \text { on } \Gamma(t), t \geqq 0 \\ V=\left[\partial_{v} u\right] & \text { on } \Gamma(t), t>0 \\ u(0, \cdot)=u_{0} & \text { in } \Omega(0) \\ \Gamma(0)=\Gamma_{0}, & \end{cases}
$$

where $\kappa(t)$ stands for the sum of the principal curvatures of $\Gamma(t)$, normalized to be positive at $x \in \Gamma(t)$ if $\Gamma(t)$ is convex at $x$ with respect to $\Omega^{-}(t)$. The normal velocity of $\Gamma$ is denoted by $V$ and is normalized to be positive at $t>0$ if $\Gamma$ is expanding locally $\Omega^{-}(t)$. Given any function $v: \Omega(t) \rightarrow \mathbb{R}$, we write $v^{+}$and $v^{-}$for the restriction of $v$ to $\Omega^{+}(t)$ and $\Omega^{-}(t)$, respectively. Using this notation, let $\left[\partial_{v} u\right]$ denote the jump of the normal derivatives of $u$ across $\Gamma(t)$, that is,

$$
\left[\partial_{v} u\right]:=\partial_{v} u^{+}-\partial_{v} u^{-}
$$

where $v$ stands for the outer normal on $\Gamma(t)$ with respect to $\Omega^{-}(t)$. Of course, $u_{0}$ is a given initial value for $u$ and $\Gamma_{0}$ describes the initial position of $\Gamma$.

To formulate our main results, let $B_{p p}^{s}\left(\mathbb{R}^{n}\right), s \geqq 0, p \in(1, \infty)$, denote the Besov spaces, cf. [51]. Then we have

Theorem 1.1. Fix $p>n+3$ and let $\rho_{0} \in B_{p p}^{4-3 / p}\left(\mathbb{R}^{n}\right)$ and $u_{0} \in B_{p p}^{2-2 / p}(\Omega(0))$ be given. Assume further that the compatibility conditions

$$
u_{0}^{ \pm}=\kappa_{\Gamma_{0}}, \quad\left[\partial_{\nu} u_{0}\right] \in B_{p p}^{2-6 / p}\left(\Gamma_{0}\right)
$$

hold. Then, given $T>0$, there exists $\varepsilon>0$ such that problem (1.3) possesses a classical solution $(\Gamma, u)$ on $[0, T]$, provided that

$$
\left\|u_{0}\right\|_{B_{p p}^{2-2 / p}(\Omega(0))}<\varepsilon, \quad\left\|\rho_{0}\right\|_{B_{p p}^{4-3 / p}\left(\mathbb{R}^{n}\right)}<\varepsilon, \quad\left\|\left[\partial_{\nu} u_{0}\right]\right\|_{B_{p p}^{2-6 / p}\left(\Gamma_{0}\right)}<\varepsilon .
$$

In addition, we have that

$$
M=\bigcup_{t \in(0, T)}(\{t\} \times \Gamma(t)) \text { is a real analytic manifold }
$$

and that $u^{ \pm} \in C^{\infty}\left(\bar{\Omega}_{T}^{ \pm}, \mathbb{R}\right)$, where $\bar{\Omega}_{T}^{ \pm}:=\left\{(t,(x, y)) \in(0, T) \times \mathbb{R}^{n+1} ;(x, y) \in \bar{\Omega}^{ \pm}(t)\right\}$.

Remarks 1.2. (a) Observe that we construct (small) smooth solutions on arbitrarily large time intervals, see also Remark (c) below. Additionally, note that $\Gamma$ is a family of real analytic hypersurfaces, depending analytically on $t \in(0, T)$.

(b) Our main results in Section 7 yield further information on the solution of Theorem 1.1. In particular, we know that the solution keeps its initial spatial regularity on the left-sided closed time interval $[0, T)$. Furthermore, the above solution is unique in an appropriate class specified in Section 7 below. 
(c) An inspection of the proof of Theorem 1.1 shows that the smallness assumption of the initial data $\left(\rho_{0}, u_{0}\right)$ can be weakened if one agrees to have solutions on (possibly) small time intervals. More precisely, one can show that there is an $\varepsilon>0$ with the following property: Given $\rho_{0} \in B_{p p}^{4-3 / p}\left(\mathbb{R}^{n}\right)$ with $\left\|\nabla \rho_{0}\right\|_{B U C\left(\mathbb{R}^{n}\right)}<\varepsilon$ and $u_{0} \in B_{p p}^{2-2 / p}(\Omega(0))$ with $u_{0}^{ \pm}=\kappa_{\Gamma_{0}}$ and $\left[\partial_{v} u_{0}\right] \in B_{p p}^{2-6 / p}\left(\Gamma_{0}\right)$, there is a $T>0$ such that problem (1.3) possesses a classical solution $(\Gamma, u)$ on $[0, T]$. This solution is again real analytic in space and time, and unique in a suitable class of functions.

(d) It is worthwhile to emphasize that the above remark in particular implies that we can handle initial geometries with arbitrarily large mean curvatures.

(e) Finally, we would like to point out that the formulation given in equation (1.3) serves as a model problem for a general geometry, as the free boundary $\Gamma(t)$ can locally always be represented as the graph of a function. It can be shown that the condition $\left\|\nabla \rho_{0}\right\|_{\infty}<\varepsilon$ can be realized by approximating $\Gamma_{0}$ by an appropriate reference manifold $\Sigma$. The smallness condition for $\nabla \rho_{0}$ will then translate into a geometric condition, expressing that the tangent space of the reference manifold $\Sigma$ be sufficiently close to the tangent space of $\Gamma_{0}$. This will be done in a forthcoming paper.

The proof of Theorem 1.1 is based on a thorough understanding of the linear problem

$$
\begin{cases}\partial_{t} w-\Delta_{x} w-\partial_{y}^{2} w=f, & t>0, x \in \mathbb{R}^{n}, y>0 \\ \gamma w+\Delta \sigma=g, & t>0, x \in \mathbb{R}^{n} \\ \partial_{t} \sigma-\gamma \partial_{y} w=h, & t>0, x \in \mathbb{R}^{n} \\ w(0, x, y)=w_{0}(x, y), & y>0, x \in \mathbb{R}^{n} \\ \sigma(0, x)=\sigma_{0}(x), & x \in \mathbb{R}^{n},\end{cases}
$$

where the initial values $\left(w_{0}, \sigma_{0}\right)$ as well as the inhomogeneities $(f, g, h)$ are given. Throughout this paper we write $\gamma$ for the (spatial) trace operator for functions defined on a domain with a smooth boundary.

We shall show that (1.4) is a parabolic problem which enjoys maximal regularity in an $L_{p}$-setting, where $1<p<\infty$ is arbitrary except for the values $3 / 2$ and 3 .

We study (1.4) in an $L_{p}$-setting, so we are using $L_{p}\left(\mathbb{R}_{+}^{n+1}\right)$ as the state space for $w$, where $\mathbb{R}_{+}^{n+1}:=\mathbb{R}^{n} \times(0, \infty)$. It turns out that the Besov space $B_{p p}^{2-2 / p}\left(\mathbb{R}^{n}\right)$ is a suitable choice for the state space of $\sigma$ in order to ensure maximal $L_{p}$-regularity for (1.4). Letting $X=L_{p}\left(\mathbb{R}_{+}^{n+1}\right) \times B_{p p}^{2-2 / p}\left(\mathbb{R}^{n}\right)$, we define the operator $A$ on $X$ by

$$
A\left(\begin{array}{c}
w \\
\sigma
\end{array}\right)=\left(\begin{array}{c}
-\Delta_{x} w-\partial_{y}^{2} w \\
-\gamma \partial_{y} w
\end{array}\right) \quad \text { for }(w, \sigma) \in \mathscr{D}(A)
$$

with

$$
\mathscr{D}(A)=\left\{(w, \sigma) \in H_{p}^{2}\left(\mathbb{R}_{+}^{n+1}\right) \times B_{p p}^{4-1 / p}\left(\mathbb{R}^{n}\right), \gamma w+\Delta \sigma=0, \gamma \partial_{y} w \in B_{p p}^{2-2 / p}\left(\mathbb{R}^{n}\right)\right\} .
$$

Our first result shows that $A$ generates an analytic $C_{0}$-semigroup on $X$. 
Theorem 1.3. Let $1<p<\infty$ and let $A$ be defined by (1.5).

Then $A$ is closed, linear and densely defined in $X$. Its kernel $\mathscr{N}(A)$ is trivial and its range $\mathscr{R}(A)$ is dense in $X$. For each $\theta \in[0, \pi)$ there is a constant $M(\theta) \geqq 1$ such that

$$
\left\|(\lambda+A)^{-1}\right\| \leqq \frac{M(\theta)}{|\lambda|}\left[1+\frac{1}{|\lambda|^{\frac{1}{2}-\frac{1}{2 p}}}\right], \quad \lambda \neq 0,|\arg \lambda| \leqq \theta .
$$

Consequently, $-A$ generates an analytic $C_{0}$-semigroup $\left\{e^{-A \zeta}\right\}_{\zeta>0}$ such that

$$
\left\|e^{-A \zeta}\right\|+\left\|\zeta A e^{-A \zeta}\right\| \leqq M_{1}(\theta)\left[1+|\zeta|^{\frac{1}{2}-\frac{1}{2 p}}\right], \quad|\arg \zeta| \leqq \theta<\pi / 2
$$

with some constant $M_{1}(\theta)$ independent of $\zeta$.

Of considerable interest are the real interpolation spaces

$$
(X ; \mathscr{D}(A))_{\alpha, q}=D_{A}(\alpha, q), \quad 1 \leqq q \leqq \infty, \alpha \in(0,1),
$$

of the domain $\mathscr{D}(A)$ of $A$, endowed with the graph norm, and $X$. For $\alpha=1-1 / p$ and $q=p$ this space characterizes all the initial values $z_{0}=\left(w_{0}, \sigma_{0}\right)$ such that $z(t)=e^{-A t} z_{0}$ belongs to the maximal regularity space of type $L_{p}$, that is,

$$
z \in H_{p}^{1}(J ; X) \cap L_{p}(J ; \mathscr{D}(A)),
$$

where $J=[0, T]$ denotes a compact interval. We refer to Remark 2.1 (b) for a complete characterization of the spaces $D_{A}(1-1 / p, p)$.

Taking into account the definition of $A$, the maximal regularity space of type $L_{p}$ according to the theory of parabolic evolution equations is given by

$$
\begin{aligned}
& w \in H_{p}^{1}\left(J ; L_{p}\left(\mathbb{R}_{+}^{n+1}\right)\right) \cap L_{p}\left(J ; H_{p}^{2}\left(\mathbb{R}_{+}^{n+1}\right)\right), \\
& \sigma \in H_{p}^{1}\left(J ; B_{p p}^{2-2 / p}\left(\mathbb{R}^{n}\right)\right) \cap L_{p}\left(J ; B_{p p}^{4-1 / p}\left(\mathbb{R}^{n}\right)\right) .
\end{aligned}
$$

However, it turns out that this regularity class is not suitable for studying the nonlinear problem. In addition, it does not take care of the inhomogeneous interface condition we have to deal with. We shall see that the appropriate maximal regularity class is given by

$$
\begin{aligned}
& w \in H_{p}^{1}\left(J ; L_{p}\left(\mathbb{R}_{+}^{n+1}\right)\right) \cap L_{p}\left(J ; H_{p}^{2}\left(\mathbb{R}_{+}^{n+1}\right)\right), \\
& \sigma \in B_{p p}^{3 / 2-1 / 2 p}\left(J ; L_{p}\left(\mathbb{R}^{n}\right)\right) \cap B_{p p}^{1-1 / 2 p}\left(J ; H_{p}^{2}\left(\mathbb{R}^{n}\right)\right) \cap L_{p}\left(J ; B_{p p}^{4-1 / p}\left(\mathbb{R}^{n}\right)\right) .
\end{aligned}
$$

Our main result concerning problem (1.4) reads as follows.

Theorem 1.4. Assume that $1<p<\infty$ with $p \neq 3 / 2,3$ and let $J=[0, T]$. Then there exists a unique solution $(w, \sigma)$ to problem $(1.4)$ with

$$
\begin{aligned}
& w \in H_{p}^{1}\left(J ; L_{p}\left(\mathbb{R}_{+}^{n+1}\right)\right) \cap L_{p}\left(J ; H_{p}^{2}\left(\mathbb{R}_{+}^{n+1}\right)\right), \\
& \sigma \in B_{p p}^{3 / 2-1 / 2 p}\left(J ; L_{p}\left(\mathbb{R}^{n}\right)\right) \cap B_{p p}^{1-1 / 2 p}\left(J ; H_{p}^{2}\left(\mathbb{R}^{n}\right)\right) \cap L_{p}\left(J ; B_{p p}^{4-1 / p}\left(\mathbb{R}^{n}\right)\right),
\end{aligned}
$$


if and only if the data $\left(f, g, h, w_{0}, \sigma_{0}\right)$ satisfy the following conditions:
(a) $f \in L_{p}\left(J ; L_{p}\left(\mathbb{R}_{+}^{n+1}\right)\right)$ and $w_{0} \in B_{p p}^{2-2 / p}\left(\mathbb{R}_{+}^{n+1}\right)$,
(b) $g \in B_{p p}^{1-1 / 2 p}\left(J ; L_{p}\left(\mathbb{R}^{n}\right)\right) \cap L_{p}\left(J ; B_{p p}^{2-1 / p}\left(\mathbb{R}^{n}\right)\right)$,
(c) $h \in B_{p p}^{1 / 2-1 / 2 p}\left(J ; L_{p}\left(\mathbb{R}^{n}\right)\right) \cap L_{p}\left(J ; B_{p p}^{1-1 / p}\left(\mathbb{R}^{n}\right)\right)$,
(d) $\sigma_{0} \in B_{p p}^{6-6 / p}\left(\mathbb{R}^{n}\right)$ for $p<3 / 2$, and $\sigma_{0} \in B_{p p}^{4-3 / p}\left(\mathbb{R}^{n}\right)$ for $p>3 / 2$,
(e) $\gamma w_{0}+\Delta \sigma_{0}=g(0)$ in case $p>3 / 2$,
(f) $\gamma \partial_{y} w_{0}+h(0) \in B_{p p}^{2-6 / p}\left(\mathbb{R}^{n}\right)$ in case $p>3$.

If this is the case then we additionally have

$$
\sigma \in H_{p}^{1}\left(J ; B_{p p}^{2-2 / p}\left(\mathbb{R}^{n}\right)\right) \cap H_{p}^{1 / 2}\left(J ; B_{p p}^{3-1 / p}\left(\mathbb{R}^{n}\right)\right) \cap B_{p p}^{1 / 2-1 / 2 p}\left(J ; H_{p}^{3}\left(\mathbb{R}^{n}\right)\right) .
$$

The maximal regularity property of (1.4) which is natural from the semigroup point of view is presented in our next result.

Theorem 1.5. Assume that $1<p<\infty$ with $p \neq 3 / 2,3$ and let $J=[0, T]$. Then there exists a unique solution $(w, \sigma)$ to problem (1.4) with

$$
\begin{aligned}
& w \in H_{p}^{1}\left(J ; L_{p}\left(\mathbb{R}_{+}^{n+1}\right)\right) \cap L_{p}\left(J ; H_{p}^{2}\left(\mathbb{R}_{+}^{n+1}\right)\right), \quad \gamma \partial_{y} w \in L_{p}\left(J ; B_{p p}^{2-2 / p}\left(\mathbb{R}^{n}\right)\right), \\
& \sigma \in H_{p}^{1}\left(J ; B_{p p}^{2-2 / p}\left(\mathbb{R}^{n}\right)\right) \cap B_{p p}^{1-1 / 2 p}\left(J ; H_{p}^{2}\left(\mathbb{R}^{n}\right)\right) \cap L_{p}\left(J ; B_{p p}^{4-1 / p}\left(\mathbb{R}^{n}\right)\right),
\end{aligned}
$$

if and only if the data $\left(f, g, h, w_{0}, \sigma_{0}\right)$ satisfy the following conditions:

(a) $f \in L_{p}\left(J ; L_{p}\left(\mathbb{R}_{+}^{n+1}\right)\right)$ and $w_{0} \in B_{p p}^{2-2 / p}\left(\mathbb{R}_{+}^{n+1}\right)$,

(b) $g \in B_{p p}^{1-1 / 2 p}\left(J ; L_{p}\left(\mathbb{R}^{n}\right)\right) \cap L_{p}\left(J ; B_{p p}^{2-1 / p}\left(\mathbb{R}^{n}\right)\right)$,

(c) $h \in L_{p}\left(J ; B_{p p}^{2-2 / p}\left(\mathbb{R}^{n}\right)\right)$,

(d) $\sigma_{0} \in B_{p p}^{6-6 / p}\left(\mathbb{R}^{n}\right)$ for $p<3 / 2$, and $\sigma_{0} \in B_{p p}^{4-3 / p}\left(\mathbb{R}^{n}\right)$ for $p>3 / 2$,

(e) $\gamma w_{0}+\Delta \sigma_{0}=g(0)$ in case $p>3 / 2$,

(f) $\gamma \partial_{y} w_{0} \in B_{p p}^{2-6 / p}\left(\mathbb{R}^{n}\right)$ in case $p>3$.

If this is the case then we additionally have

$$
\sigma \in H_{p}^{1 / 2}\left(J ; B_{p p}^{3-1 / p}\left(\mathbb{R}^{n}\right)\right) \cap B_{p p}^{1 / 2-1 / 2 p}\left(J ; H_{p}^{3}\left(\mathbb{R}^{n}\right)\right) .
$$

The plan for this paper is as follows. Section 2 contains the construction of the semigroup, that is, the proof of Theorem 1.3. In Section 3 we study $L_{p}$-maximal regularity for problem (1.4) in the case that $w_{0}=\sigma_{0}=0$ and $g \equiv 0$, whereas the case $w_{0}=\sigma_{0}=0$ and 
$f \equiv h \equiv 0$ is considered in Section 4. The proofs of Theorems 1.4 and 1.5 are given in Section 5. Section 6 is devoted to a short discussion of the corresponding two-phase problem and Theorem 6.1 is the analogue of Theorem 1.4 for the two-phase case. Based on Theorem 6.1 we prove in Section 7 existence and uniqueness of $L_{p}$-solutions for the nonlinear equation (1.3). Section 8 is devoted to establishing the spatial and temporal analyticity of the free interface. Here again, maximal regularity is of crucial importance. Finally, Section 9 contains some technical results that are needed in the main body of the paper.

\section{The semigroup in the $L_{p}$-setting}

In this section we prove Theorem 1.3. In order to show that $A$ generates a $C_{0}$ semigroup which is even analytic on each sector $\Sigma_{\varphi}=\{z \in \mathbb{C}: z \neq 0,|\arg z|<\varphi\}, \varphi<\pi / 2$, we have to compute and estimate the resolvent of $A$. For this purpose we first solve the resolvent problem

$$
\begin{cases}\lambda w-\Delta_{x} w-\partial_{y}^{2} w=w_{0}, & x \in \mathbb{R}^{n}, y>0, \\ \gamma w+\Delta \sigma=0, & x \in \mathbb{R}^{n}, \\ \lambda \sigma-\gamma \partial_{y} w=\sigma_{0}, & x \in \mathbb{R}^{n} .\end{cases}
$$

Taking the Fourier transform in $x$ leads to an ODE-problem for $u(y, \xi)=\tilde{w}(\xi, y)$ and $\rho(\xi)=\tilde{\sigma}(\xi)$, namely

$$
\left\{\begin{array}{l}
\left(\lambda+|\xi|^{2}\right) u-\partial_{y}^{2} u=u_{0}, \quad y>0 \\
u(0)=|\xi|^{2} \rho \\
\lambda \rho-\partial_{y} u(0)=\rho_{0}
\end{array}\right.
$$

This is easily solved to the result

$$
\begin{aligned}
u(y, \xi) & =\int_{0}^{\infty} \frac{e^{-\omega|y-s|}}{2 \omega} u_{0}(s) d s+\frac{\omega|\xi|^{2}-\lambda}{\omega|\xi|^{2}+\lambda} \int_{0}^{\infty} \frac{e^{-\omega(y+s)}}{2 \omega} u_{0}(s) d s+e^{-\omega y} \frac{|\xi|^{2} \rho_{0}(\xi)}{\lambda+\omega|\xi|^{2}} \\
\rho(\xi) & =\frac{\rho_{0}(\xi)}{\lambda+\omega|\xi|^{2}}+\frac{1}{\lambda+\omega|\xi|^{2}} \int_{0}^{\infty} e^{-\omega s} u_{0}(s) d s
\end{aligned}
$$

Here and in the following we use the abbreviation

$$
\omega=\sqrt{\lambda+|\xi|^{2}}
$$

Let us analyze the structure of $(\lambda+A)^{-1}$ in some detail.

$$
(\lambda+A)^{-1}=\left[\begin{array}{ll}
R_{11}(\lambda) & R_{12}(\lambda) \\
R_{21}(\lambda) & R_{22}(\lambda)
\end{array}\right],
$$

with the following entries $R_{i j}(\lambda)$ :

$$
R_{11}(\lambda)=P_{+}\left(\lambda+D_{n+1}\right)^{-1} E_{0}+P_{+} \hat{S}(\lambda) R\left(\lambda+D_{n+1}\right)^{-1} E_{0} .
$$

Here $E_{0}$ denotes the operator of extension by $0 ; E_{0} \in \mathscr{B}\left(L_{p}\left(\mathbb{R}_{+}^{n+1}\right) ; L_{p}\left(\mathbb{R}^{n+1}\right)\right)$ is an isomet- 
ric and linear operator. $P_{+} \in \mathscr{B}\left(L_{p}\left(\mathbb{R}^{n+1}\right) ; L_{p}\left(\mathbb{R}_{+}^{n+1}\right)\right)$ denotes the restriction, hence $P_{+}^{2}=P_{+}$ and $\left\|P_{+}\right\|=1$. By $R$ we mean the reflection w.r.t. $y$, that is,

$$
(R f)(x, y)=f(x,-y)
$$

which is linear and isometric in $L_{p}\left(\mathbb{R}^{n+1}\right)$, and $R^{2}=I$, the identity. $D_{m}$ denotes $-\Delta$ on $L_{p}\left(\mathbb{R}^{m}\right)$ with domain $H_{p}^{2}\left(\mathbb{R}^{m}\right)$. It is well-known that $-D_{m}$ generates a $C_{0}$-semigroup which is analytic and bounded in each sector $\Sigma_{\varphi}$. In particular, the resolvent estimate

$$
\left\|\left(\lambda+D_{m}\right)^{-1}\right\|_{\mathscr{B}\left(L_{p}\left(\mathbb{R}^{m}\right)\right)} \leqq \frac{C_{\theta}}{|\lambda|}, \quad \lambda \in \Sigma_{\theta},
$$

holds for each $\theta<\pi$. Finally, $\hat{S}(\lambda)$ is a singular integral operator with symbol

$$
\frac{\omega|\xi|^{2}-\lambda}{\omega|\xi|^{2}+\lambda}=1-2 \frac{\lambda}{\lambda+\omega|\xi|^{2}} .
$$

Since by Lemma 9.1 the Mikhlin condition is satisfied for this symbol, uniformly in $\lambda \in \Sigma_{\theta}$, $\theta<\pi, \hat{S}(\lambda)$ is bounded in $H_{p}^{s}\left(\mathbb{R}^{n}\right)$ for each $s, 1<p<\infty$, hence also in $L_{p}\left(\mathbb{R}_{+}^{n+1}\right)$ by canonical extension. The bound is uniform in $\lambda$, it depends only on $p, n, \theta$.

Next, $R_{22}(\lambda)$ is an integral operator of convolution type with symbol $\left(\lambda+\omega|\xi|^{2}\right)^{-1}$, acting on $\mathbb{R}^{n}$. Again by Lemma 9.1 , it is bounded in each space $H_{p}^{s}\left(\mathbb{R}^{n}\right)$, with resolvent estimate

$$
\left\|R_{22}(\lambda)\right\|_{\mathscr{B}\left(H_{p}^{s}\left(\mathbb{R}^{n}\right)\right)} \leqq \frac{C_{\theta}}{|\lambda|}, \quad \lambda \in \Sigma_{\theta}
$$

hence by real interpolation we also have

$$
\left\|R_{22}(\lambda)\right\|_{\mathscr{B}\left(B_{p q}^{s}\left(\mathbb{R}^{n}\right)\right)} \leqq \frac{C_{\theta}}{|\lambda|}, \quad \lambda \in \Sigma_{\theta} .
$$

Moreover, again by Lemma 9.1

$$
\left\|D_{n}^{3 / 2} R_{22}(\lambda)\right\|_{\mathscr{B}\left(H_{p}^{s}\left(\mathbb{R}^{n}\right)\right)} \leqq C_{\theta}, \quad \lambda \in \Sigma_{\theta},
$$

as well as

$$
\left\|D_{n} R_{22}(\lambda)\right\|_{\mathscr{B}\left(H_{p}^{s}\left(\mathbb{R}^{n}\right)\right)} \leqq \frac{C_{\theta}}{\sqrt{|\lambda|}}, \quad \lambda \in \Sigma_{\theta} .
$$

Thus interpolation yields

$$
\left\|D_{n}^{3 / 2} R_{22}(\lambda)\right\|_{\mathscr{B}\left(B_{p q}^{s}\left(\mathbb{R}^{n}\right)\right)} \leqq C_{\theta}, \quad \lambda \in \Sigma_{\theta},
$$

and

$$
\left\|D_{n} R_{22}(\lambda)\right\|_{\mathscr{B}\left(B_{p q}^{s}\left(\mathbb{R}^{n}\right)\right)} \leqq \frac{C_{\theta}}{\sqrt{|\lambda|}}, \quad \lambda \in \Sigma_{\theta} .
$$


So far, we have shown that the diagonal entries map into the domain of $A$ and satisfy the resolvent estimate aimed at.

The proper choice of the phase space for $\sigma$ will become apparent when we consider the off-diagonal entries $R_{12}(\lambda)$ and $R_{21}(\lambda)$. We have

$$
R_{12}(\lambda)=\hat{E}(\lambda) D_{n} R_{22}(\lambda)
$$

and

$$
R_{21}(\lambda)=R_{22}(\lambda) \hat{E}^{*}(\lambda)
$$

where $\hat{E}(\lambda)$ denotes the Dirichlet extension operator with symbol $e^{-\omega y}$, that is,

$$
(\hat{E}(\lambda) \varphi)^{\sim}(y, \xi)=e^{-\omega y} \tilde{\varphi}(\xi), \quad \xi \in \mathbb{R}^{n}, y>0,
$$

and where $\hat{E}^{*}(\lambda)$ is given by

$$
\left(\hat{E}^{*}(\lambda) v\right)^{\sim}(\xi)=\int_{0}^{\infty} e^{-\omega y} \tilde{v}(y, \xi) d y .
$$

The mapping properties of $\hat{E}(\lambda)$ and $\hat{E}^{*}(\lambda)$ are also well-known, but we do not hesitate to derive them here, for the sake of completeness.

For this purpose we define $\hat{E}_{y}(\lambda)$ by the symbol $e^{-\omega y}$. Then Lemma 9.3 yields $\hat{E}_{y}(\lambda) \in \mathscr{B}\left(H_{p}^{s}\left(\mathbb{R}^{n}\right)\right)$ and

$$
\left\|\hat{E}_{y}(\lambda)\right\|_{\mathscr{B}\left(H_{p}^{s}\left(\mathbb{R}^{n}\right)\right)} \leqq C_{\theta} e^{-c_{\theta} y \sqrt{|\lambda|}}, \quad y>0,|\arg \lambda|<\theta<\pi,
$$

for $s \in \mathbb{R}$, where $C_{\theta}$ and $c_{\theta}$ are positive constants. This implies

$$
\begin{aligned}
\left\|R_{12}(\lambda) \varphi\right\|_{L_{p}\left(\mathbb{R}_{+}^{n+1}\right)}^{p} & =\int_{0}^{\infty}\left\|\hat{E}_{y}(\lambda) D_{n} R_{22}(\lambda) \varphi\right\|_{L_{p}\left(\mathbb{R}^{n}\right)}^{p} d y \\
& \leqq C_{\theta} \int_{0}^{\infty} e^{-c_{\theta} y \sqrt{|\lambda|}}\left\|D_{n} R_{22}(\lambda) \varphi\right\|_{L_{p}\left(\mathbb{R}^{n}\right)}^{p} d y \\
& =\frac{M_{\theta}}{|\lambda|^{1 / 2}}\left\|D_{n} R_{22}(\lambda)\right\|_{\mathscr{B}\left(B_{p p}^{2-2 / p}\left(\mathbb{R}^{n}\right), L_{p}\left(\mathbb{R}^{n}\right)\right)}^{p}\|\varphi\|_{B_{p p}^{2-2 / p}\left(\mathbb{R}^{n}\right)}^{p} \\
& \leqq \frac{M_{\theta}}{|\lambda|^{p}}\|\varphi\|_{B_{p p}^{2-2 / p}\left(\mathbb{R}^{n}\right)}^{p},
\end{aligned}
$$

since we have by $(2.4)$

$$
\left\|D_{n} R_{22}(\lambda)\right\|_{\mathscr{B}\left(H_{p}^{2}\left(\mathbb{R}^{n}\right), L_{p}\left(\mathbb{R}^{n}\right)\right)} \leqq \frac{C_{\theta}}{|\lambda|},
$$

and by (2.7)

$$
\left\|D_{n} R_{22}(\lambda)\right\|_{\mathscr{B}\left(L_{p}\left(\mathbb{R}^{n}\right), L_{p}\left(\mathbb{R}^{n}\right)\right)} \leqq \frac{C_{\theta}}{\sqrt{|\lambda|}},
$$


and hence by interpolation

$$
\left\|D_{n} R_{22}(\lambda)\right\|_{\mathscr{B}\left(B_{p p}^{2-2 / p}\left(\mathbb{R}^{n}\right), L_{p}\left(\mathbb{R}^{n}\right)\right)} \leqq \frac{C_{\theta}}{|\lambda|^{1-1 / 2 p}} .
$$

This shows that entry $R_{12}(\lambda)$ satisfies the resolvent estimate.

Next we consider $\hat{E}^{*}(\lambda)$. We have

$$
\begin{aligned}
\left\|\hat{E}^{*}(\lambda) f\right\|_{L_{p}\left(\mathbb{R}^{n}\right)} & =\left\|\int_{0}^{\infty} \hat{E}_{y}(\lambda) f(y, \cdot) d y\right\|_{L_{p}\left(\mathbb{R}^{n}\right)} \\
& \leqq \int_{0}^{\infty}\left\|\hat{E}_{y}(\lambda)\right\|_{\mathscr{B}\left(L_{p}\left(\mathbb{R}^{n}\right)\right)}\|f(y, \cdot)\|_{L_{p}\left(\mathbb{R}^{n}\right)} d y \\
& \leqq C_{\theta} \int_{0}^{\infty} e^{-c_{\theta} y \sqrt{|\lambda|}}\|f(y, \cdot)\|_{L_{p}\left(\mathbb{R}^{n}\right)} d y \\
& \leqq M_{\theta} \frac{1}{|\lambda|^{1 / 2-1 / 2 p}}\|f\|_{L_{p}\left(\mathbb{R}_{+}^{n+1}\right)},
\end{aligned}
$$

by Hölder's inequality and the estimate for $\hat{E}_{y}(\lambda)$. Interpolating (2.4) and (2.7) we obtain

$$
\left\|R_{22}(\lambda)\right\|_{\mathscr{B}\left(L_{p}\left(\mathbb{R}^{n}\right), B_{p p}^{2-2 / p}\left(\mathbb{R}^{n}\right)\right)} \leqq C_{\theta}\left[\frac{1}{|\lambda|^{\left[1 / p+\frac{1}{2}(1-1 / p)\right]}}+\frac{1}{|\lambda|}\right], \quad \lambda \in \Sigma_{\theta},
$$

hence

$$
\left\|R_{21}(\lambda)\right\|_{\mathscr{B}\left(L_{p}\left(\mathbb{R}_{+}^{n+1}\right), B_{p p}^{2-2 / p}\left(\mathbb{R}^{n}\right)\right)} \leqq M_{\theta} C_{\theta} \frac{1}{|\lambda|}\left[1+\frac{1}{|\lambda|^{1 / 2-1 / 2 p}}\right], \quad \lambda \in \Sigma_{\theta},
$$

i.e. $R_{21}(\lambda)$ fulfills the resolvent estimate for large $\lambda$ as well.

For fixed $\lambda$, the family $\left\{\hat{E}_{y}(\lambda)\right\}_{y \geqq 0}$ forms a $C_{0}$-semigroup on $L_{p}\left(\mathbb{R}^{n}\right)$. Its negative generator $B$ has the symbol $\omega=\sqrt{\lambda+|\xi|^{2}}$ and we conclude that $\mathscr{D}(B)=H_{p}^{1}\left(\mathbb{R}^{n}\right)$. Therefore the real interpolation spaces $D_{B}(\alpha, q)$ are

$$
D_{B}(\alpha, q) \cong B_{p q}^{\alpha}\left(\mathbb{R}^{n}\right), \quad \alpha \in(0,1), q \in[1, \infty] .
$$

From this we conclude that $\hat{E}^{*}(\lambda) \in \mathscr{B}\left(L_{p}\left(\mathbb{R}_{+}^{n+1}\right), B_{p p}^{1-1 / p}\left(\mathbb{R}^{n}\right)\right)$ as follows.

$$
\begin{aligned}
\left\|\hat{E}^{*}(\lambda) f\right\|_{B_{p p}^{1-1 / p}\left(\mathbb{R}^{n}\right)}^{p} & \leqq C_{1} \int_{0}^{\infty}\left[t^{1-(1-1 / p)}\left\|B \hat{E}_{t}(\lambda) \hat{E}^{*}(\lambda) f\right\|_{L_{p}\left(\mathbb{R}^{n}\right)}\right]^{p} \frac{d t}{t} \\
& \leqq C_{1} \int_{0}^{\infty}\left\|\int_{0}^{\infty} B \hat{E}_{t+y}(\lambda) f(y, \cdot) d y\right\|_{L_{p}\left(\mathbb{R}^{n}\right)}^{p} d t \\
& \leqq C_{2} \int_{0}^{\infty}\left[\int_{0}^{\infty} \frac{\|f(y, \cdot)\|_{L_{p}\left(\mathbb{R}^{n}\right)}}{y+t} d y\right]^{p} d t \leqq C_{3}\|f\|_{L_{p}\left(\mathbb{R}_{+}^{n+1}\right)}^{p}
\end{aligned}
$$

by Hilbert's inequality which is stated in Lemma 9.4. 
By equation (2.6) and (2.4) we have

$$
R_{22}(\lambda) \in \mathscr{B}\left(B_{p p}^{1-1 / p}\left(\mathbb{R}^{n}\right), B_{p p}^{4-1 / p}\left(\mathbb{R}^{n}\right)\right)
$$

hence

$$
R_{21}(\lambda) \in \mathscr{B}\left(L_{p}\left(\mathbb{R}_{+}^{n+1}\right), B_{p p}^{4-1 / p}\left(\mathbb{R}^{n}\right)\right)
$$

Estimate (2.8) yields $D_{n} R_{22}(\lambda) \in \mathscr{B}\left(B_{p q}^{s}\left(\mathbb{R}^{n}\right), B_{p q}^{s+1}\left(\mathbb{R}^{n}\right)\right)$, and therefore, given $\varphi \in B_{p p}^{2-2 / p}\left(\mathbb{R}^{n}\right)$, we have $D_{n} R_{22}(\lambda) \varphi \in B_{p p}^{3-2 / p}\left(\mathbb{R}^{n}\right)$, and

$$
B D_{n} R_{22}(\lambda) \varphi \in B_{p p}^{2-2 / p}\left(\mathbb{R}^{n}\right) \subset B_{p p}^{1-1 / p}\left(\mathbb{R}^{n}\right)=D_{B}(1-1 / p, p) .
$$

Since $D_{n} B^{-2}$ is bounded by Lemma 9.2 , this implies

$$
\begin{aligned}
\left\|D_{n} R_{12}(\lambda) \varphi\right\|_{L^{p}\left(\mathbb{R}_{+}^{n+1}\right)}^{p} & =\int_{0}^{\infty}\left\|D_{n} \hat{E}_{y}(\lambda) D_{n} R_{22}(\lambda)\right\|_{L^{p}\left(\mathbb{R}^{n}\right)}^{p} d y \\
& \leqq\left\|D_{n} B^{-2}\right\|_{\mathscr{B}\left(L^{p}\left(\mathbb{R}^{n}\right)\right)}^{p} \int_{0}^{\infty}\left\|B \hat{E}_{y}(\lambda) B D_{n} R_{22}(\lambda) \varphi\right\|_{L^{p}\left(\mathbb{R}^{n}\right)}^{p} d y \\
& \leqq C\|\varphi\|_{B_{p p}^{1-1 / p}\left(\mathbb{R}^{n}\right)}^{p}
\end{aligned}
$$

Thus $R_{12}(\lambda) \varphi \in H_{p}^{2}\left(\mathbb{R}_{+}^{n+1}\right)$.

By construction of the resolvent we have

$$
\gamma \partial_{y} w=\lambda \sigma-\sigma_{0}
$$

hence $\gamma \partial_{y} w \in B_{p p}^{2-2 / p}\left(\mathbb{R}^{n}\right)$. Also $\Delta \sigma \in B_{p p}^{2-1 / p}\left(\mathbb{R}^{n}\right)$, and by the trace theorem we obtain that $\gamma w \in B_{p p}^{2-1 / p}\left(\mathbb{R}^{n}\right)$ as well, and the identity

$$
\gamma w+\Delta \sigma=0
$$

holds by construction and uniqueness of the Fourier transform.

It follows from the fact that $X$ is reflexive and from the resolvent estimate near infinity that $\mathscr{D}(A)$ is dense in $X$. The kernel of $A$ is trivial, because the Neumann problem

$$
\begin{gathered}
\Delta_{x} w+\partial_{y}^{2} w=0, \quad x \in \mathbb{R}^{n}, y>0, \\
\gamma \partial_{y} w=0, \quad x \in \mathbb{R}^{n},
\end{gathered}
$$

admits only the zero solution in $L_{p}\left(\mathbb{R}_{+}^{n+1}\right)$, and the equation $\Delta_{x} \sigma=0$ in $\mathbb{R}^{n}$ has only the zero solution in $L_{p}\left(\mathbb{R}^{n}\right)$. It is not difficult to see that 0 belongs to the continuous spectrum of $A$. Therefore, the inverse of $A$ is unbounded.

Remarks 2.1. (a) It is not clear whether the estimate $\left\|R_{21}(\lambda)\right\| \leqq C /|\lambda|$ is valid, that is, whether the semigroup $e^{-A t}$ is uniformly bounded. This would be the case if we had 
worked with homogeneous Besov spaces instead of inhomogeneous ones. We prefer the latter due to the application of the linear theory to the nonlinear problem, cf. Remark 5.1.3.2 in [51].

(b) As a consequence of the methods employed in Section 5 we obtain a complete characterization of the interpolation spaces $D_{A}(1-1 / p, p)$, namely

$$
\begin{gathered}
D_{A}(1-1 / p, p)=\left\{\left(w_{0}, \sigma_{0}\right) \in X ; w_{0} \in B_{p p}^{2-2 / p}\left(\mathbb{R}_{+}^{n+1}\right), \sigma_{0} \in B_{p p}^{4-3 / p}\left(\mathbb{R}^{n}\right)\right. \\
\left.\gamma w_{0}+\Delta \sigma_{0}=0, \gamma \partial_{y} w_{0} \in B_{p p}^{2-6 / p}\left(\mathbb{R}^{n}\right)\right\} .
\end{gathered}
$$

This characterization is valid for $p>3$. The second compatibility condition is void for $p \in(3 / 2,3)$, and for $p<3 / 2$ the first one disappears. We then have

$$
D_{A}(1-1 / p, p)=\left\{\left(w_{0}, \sigma_{0}\right) \in X ; w_{0} \in B_{p p}^{2-2 / p}\left(\mathbb{R}_{+}^{n+1}\right), \sigma_{0} \in B_{p p}^{6-6 / p}\left(\mathbb{R}^{n}\right)\right\} .
$$

The cases $p=3 / 2$ and $p=3$ are excluded here.

\section{Maximal regularity of type $L_{p}$}

We now study problem (1.4) in the case that $w_{0}=\sigma_{0}=0$ and $g \equiv 0$. We show that this problem enjoys the property of maximal $L_{p}$-regularity. This will be achieved by a combination of the Dore-Venni theorem and results about the Dirichlet extension operator which rely on real interpolation techniques.

Proposition 3.1. Let $1<p<\infty$ and let $(w, \sigma):=e^{-A t} *(f, g)$. that

Then $f \in L_{p}\left(J ; L_{p}\left(\mathbb{R}_{+}^{n+1}\right)\right)$ and $h \in{ }_{0} B_{p p}^{1 / 2-1 / 2 p}\left(J ; L_{p}\left(\mathbb{R}^{n}\right)\right) \cap L_{p}\left(J ; B_{p p}^{1-1 / p}\left(\mathbb{R}^{n}\right)\right)$ implies

$$
\begin{aligned}
& w \in{ }_{0} H_{p}^{1}\left(J ; L_{p}\left(\mathbb{R}_{+}^{n+1}\right)\right) \cap L_{p}\left(J ; H_{p}^{2}\left(\mathbb{R}_{+}^{n+1}\right)\right), \\
& \sigma \in{ }_{0} B_{p p}^{3 / 2-1 / 2 p}\left(J ; L_{p}\left(\mathbb{R}^{n}\right)\right) \cap{ }_{0} B_{p p}^{1-1 / 2 p}\left(J ; H_{p}^{2}\left(\mathbb{R}^{n}\right)\right) \cap L_{p}\left(J ; B_{p p}^{4-1 / p}\left(\mathbb{R}^{n}\right)\right)
\end{aligned}
$$

as well as

$$
\sigma \in{ }_{0} H_{p}^{1}\left(J ; B_{p p}^{2-2 / p}\left(\mathbb{R}^{n}\right)\right) \cap_{0} H_{p}^{1 / 2}\left(J ; B_{p p}^{3-1 / p}\left(\mathbb{R}^{n}\right)\right) \cap_{0} B_{p p}^{1 / 2-1 / 2 p}\left(J ; H_{p}^{3}\left(\mathbb{R}^{n}\right)\right) .
$$

Here we use the notation

$$
{ }_{0} B_{p q}^{s}(J ; Y):= \begin{cases}\left\{u \in B_{p q}^{s}(J ; Y): u^{\prime}(0)=u(0)=0\right\}, & 1+\frac{1}{p}<s<2+\frac{1}{p} \\ \left\{u \in B_{p q}^{s}(J ; Y): u(0)=0\right\}, & \frac{1}{p}<s<1+\frac{1}{p} \\ B_{p q}^{s}(J ; Y), & s<\frac{1}{p},\end{cases}
$$

where $Y$ means an arbitrary Banach space. The spaces ${ }_{0} H_{p}^{s}(J ; Y)$ are defined analogously. 
Proof. Let $J=[0, T]$ be a compact interval. Let us introduce some operators and interpret the symbol representation of the resolvent of $A$, defined in Section 3 , in terms of these operators. For a Banach space $Y$ we first let

$$
(G u)(t)=\frac{d}{d t} u(t), \quad t \in J, u \in \mathscr{D}(G)
$$

with

$$
\mathscr{D}(G)={ }_{0} H_{p}^{1}(J ; Y)=\left\{u \in H_{p}^{1}(J ; Y): u(0)=0\right\} \subset L_{p}(J ; Y)=: X .
$$

$G$ is closed, linear, invertible with spectral angle $\varphi_{G}=\pi / 2$. Moreover, $G$ admits bounded imaginary powers, $G \in B I P(X)$, if $Y$ is a space of class $\mathscr{H} \mathscr{T}$. The power angle of $G$ is $\theta_{G}=\pi / 2$; see e.g. Prüss [42].

Next let $Y=H_{p}^{s}\left(\mathbb{R}^{n}\right)$ for some $s$, and denote by $D_{n}$ the canonical extension of $-\Delta$ to $L_{p}\left(J ; H_{p}^{s}\left(\mathbb{R}^{n}\right)\right)$. It is well-known that $D_{n}$ is sectorial with spectral angle $\varphi_{D_{n}}=0$. Moreover, $D_{n}$ admits bounded imaginary powers, that is, $D_{n} \in B I P(X)$ with $\theta_{D_{n}}=0$. Since $G$ and $D_{n}$ commute, by the Dore-Venni theorem in the form given in Prüss and Sohr [44], $G+D_{n}$ is closed, invertible with natural domain $\mathscr{D}\left(G+D_{n}\right)=\mathscr{D}(G) \cap \mathscr{D}\left(D_{n}\right)$, i.e.

$$
\mathscr{D}\left(G+D_{n}\right)={ }_{0} H_{p}^{1}\left(J ; H_{p}^{s}\left(\mathbb{R}^{n}\right)\right) \cap L_{p}\left(J ; H_{p}^{s+2}\left(\mathbb{R}^{n}\right)\right) .
$$

Moreover, $G+D_{n}$ is sectorial and belongs to $B I P(X)$ with $\varphi_{G+D_{n}} \leqq \theta_{G+D_{n}} \leqq \pi / 2$. Therefore, also $F:=\left(G+D_{n}\right)^{1 / 2} \in B I P(X)$ and $\theta_{F} \leqq \pi / 4$, using Prüss and Sohr [44] once more. Since $F$ and $D_{n}$ commute, $F$ is invertible, we obtain $F D_{n} \in B I P(X)$ and $\theta_{F D_{n}} \leqq \pi / 4$, with natural domain

$$
\mathscr{D}\left(F D_{n}\right)=\left\{u \in \mathscr{D}\left(D_{n}\right): D_{n} u \in \mathscr{D}(F)\right\}
$$

Finally, applying the Dore-Venni theorem another time we obtain that

$$
L:=G+F D_{n}=G+\left(G+D_{n}\right)^{1 / 2} D_{n}
$$

is closed with natural domain $\mathscr{D}(L)=\mathscr{D}(G) \cap \mathscr{D}\left(F D_{n}\right)$ and invertible, as well as sectorial, $L \in B I P(X)$ with $\varphi_{L} \leqq \theta_{L} \leqq \pi / 2$. To determine the domain of $L$, we have, using complex interpolation and Lemma 9.5,

$$
\begin{aligned}
\mathscr{D}(F) & =\mathscr{D}\left(\left(G+D_{n}\right)^{1 / 2}\right)=\left[X, \mathscr{D}(G) \cap \mathscr{D}\left(D_{n}\right)\right]_{1 / 2} \\
& ={ }_{0} H_{p}^{1 / 2}\left(J ; H_{p}^{s}\left(\mathbb{R}^{n}\right)\right) \cap L_{p}\left(J ; H_{p}^{s+1}\left(\mathbb{R}^{n}\right)\right) .
\end{aligned}
$$

This then gives

$$
\begin{aligned}
\mathscr{D}(L) & =\mathscr{D}(G) \cap \mathscr{D}\left(F D_{n}\right) \\
& ={ }_{0} H_{p}^{1}\left(J ; H_{p}^{s}\left(\mathbb{R}^{n}\right)\right) \cap{ }_{0} H_{p}^{1 / 2}\left(J ; H_{p}^{s+2}\left(\mathbb{R}^{n}\right)\right) \cap L_{p}\left(J ; H_{p}^{s+3}\left(\mathbb{R}^{n}\right)\right),
\end{aligned}
$$

the space of maximal regularity for the symbol $\left(\lambda+\omega|\xi|^{2}\right)^{-1}$. By means of real interpola- 
tion we obtain from this also the maximal regularity class when the underlying space is $B_{p p}^{s}\left(\mathbb{R}^{n}\right)$ instead of $H_{p}^{s}\left(\mathbb{R}^{n}\right)$. It is

$$
{ }_{0} H_{p}^{1}\left(J ; B_{p p}^{s}\left(\mathbb{R}^{n}\right)\right) \cap{ }_{0} H_{p}^{1 / 2}\left(J ; B_{p p}^{s+2}\left(\mathbb{R}^{n}\right)\right) \cap L_{p}\left(J ; B_{p p}^{s+3}\left(\mathbb{R}^{n}\right)\right) .
$$

Next we treat the first entry of the resolvent of $A$. Extending the operators $P_{+}, E_{0}, R$ and $D_{n+1}$ in the canonical way to $X_{1}:=L_{p}\left(J ; L_{p}\left(\mathbb{R}_{+}^{n+1}\right)\right)$ we obtain for the first entry of $(G+A)^{-1}$

$$
(G+A)_{11}^{-1}=P_{+}\left(G+D_{n+1}\right)^{-1} E_{0}+P_{+} R\left(G+D_{n+1}\right)^{-1}\left(I-2 G L^{-1}\right) E_{0} .
$$

Since $E_{0}, G L^{-1} \in \mathscr{B}\left(X_{1}\right), \mathscr{D}\left(G+D_{n+1}\right)=\mathscr{D}(G) \cap \mathscr{D}\left(D_{n+1}\right)$, i.e.

$$
\mathscr{R}\left(\left(G+D_{n+1}\right)^{-1}\right)=\mathscr{D}\left(G+D_{n+1}\right)={ }_{0} H_{p}^{1}\left(J ; L_{p}\left(\mathbb{R}_{+}^{n+1}\right)\right) \cap L_{p}\left(J ; H_{p}^{2}\left(\mathbb{R}_{+}^{n+1}\right)\right) .
$$

The reflection $R$ and the projection $P_{+}$preserve this regularity. We therefore obtain

$$
\mathscr{R}\left((G+A)_{11}^{-1}\right) \subset{ }_{0} H_{p}^{1}\left(J ; L_{p}\left(\mathbb{R}_{+}^{n+1}\right)\right) \cap L_{p}\left(J ; H_{p}^{2}\left(\mathbb{R}_{+}^{n+1}\right)\right),
$$

that is, the $w$-part coming from $f$ has the desired optimal regularity behavior.

To study the entry $(G+A)_{21}^{-1}$, let $f \in X_{1}=L_{p}\left(J ; L_{p}\left(\mathbb{R}_{+}^{n+1}\right)\right)$ be given. Since $F$ is sectorial with spectral angle $\varphi_{F} \leqq \pi / 4,-F$ generates a bounded analytic $C_{0}$-semigroup $e^{-F y}$ on $X_{0}=L_{p}\left(J ; L_{p}\left(\mathbb{R}^{n+1}\right)\right)$. We may then write

$$
(G+A)_{21}^{-1} f=L^{-1} \int_{0}^{\infty} e^{-F y} f(y) d y,
$$

where we consider $f \in L_{p}\left(\mathbb{R}_{+} ; L_{p}\left(J ; L_{p}\left(\mathbb{R}^{n}\right)\right)\right) \cong X_{1}$. To see the regularity involved, we calculate

$$
\begin{gathered}
\int_{0}^{\infty}\left\|t^{1-(1-1 / p)} F e^{-F t} \int_{0}^{\infty} e^{-F y} f(y) d y\right\|^{p} \frac{d t}{t}=\int_{0}^{\infty}\left\|\int_{0}^{\infty} F e^{-F(t+y)} f(y) d y\right\|^{p} d t \\
\leqq M_{0} \int_{0}^{\infty}\left[\int_{0}^{\infty} \frac{\|f(y)\|}{y+t} d y\right]^{p} d t \leqq M_{1} \int_{0}^{\infty}\|f(y)\|^{p} d y=M_{1}\|f\|_{X_{1}}^{p},
\end{gathered}
$$

that is,

$$
\varphi:=\int_{0}^{\infty} e^{-y F} f(y) d y \in D_{F}(1-1 / p, p),
$$

a trace space for $F$. With $X_{0}=L_{p}\left(J ; L_{p}\left(\mathbb{R}^{n}\right)\right)$ one obtains by Lemma 9.6

$$
\begin{aligned}
D_{F}(1-1 / p, p) & =\left(X_{0}, \mathscr{D}(F)\right)_{1-1 / p, p}=\left(X_{0}, \mathscr{D}\left(G^{1 / 2}\right) \cap \mathscr{D}\left(D_{n}^{1 / 2}\right)\right)_{1-1 / p, p} \\
& =\left(X_{0}, \mathscr{D}\left(G^{1 / 2}\right)\right)_{1-1 / p, p} \cap\left(X_{0}, \mathscr{D}\left(D_{n}^{1 / 2}\right)\right)_{1-1 / p, p} \\
& ={ }_{0} B_{p p}^{1 / 2-1 / 2 p}\left(J ; L_{p}\left(\mathbb{R}^{n}\right)\right) \cap L_{p}\left(J ; B_{p p}^{1-1 / p}\left(\mathbb{R}^{n}\right)\right) .
\end{aligned}
$$


By choosing $f(y)=2 F e^{-F y} \varphi$ we immediately see that the map $f \mapsto \varphi$ is surjective, hence this regularity of $\varphi=\int_{0}^{\infty} e^{-F y} f(y) d y$ is optimal.

Applying the mapping properties of $L^{-1}$ with $s=1-1 / p$, we see that $\mathscr{R}\left((G+A)_{21}^{-1}\right)$ is contained in

$$
{ }_{0} H_{p}^{1}\left(J ; B_{p p}^{1-1 / p}\left(\mathbb{R}^{n}\right)\right) \cap{ }_{0} H_{p}^{1 / 2}\left(J ; B_{p p}^{3-1 / p}\left(\mathbb{R}^{n}\right)\right) \cap L_{p}\left(J ; B_{p p}^{4-1 / p}\left(\mathbb{R}^{n}\right)\right) .
$$

But there is more time regularity involved, coming from $\varphi \in{ }_{0} B_{p p}^{1 / 2-1 / 2 p}\left(J ; L_{p}\left(\mathbb{R}^{n}\right)\right)$. In fact, we may repeat the argument for $L$ in ${ }_{0} H_{p}^{r}\left(J ; H_{p}^{s}\left(\mathbb{R}^{n}\right)\right)$, to obtain for this space

$$
\mathscr{D}(L)={ }_{0} H_{p}^{r+1}\left(J ; H_{p}^{s}\left(\mathbb{R}^{n}\right)\right) \cap{ }_{0} H_{p}^{r+1 / 2}\left(J ; H_{p}^{s+2}\left(\mathbb{R}^{n}\right)\right) \cap_{0} H_{p}^{r}\left(J ; H_{p}^{s+3}\left(\mathbb{R}^{n}\right)\right),
$$

and then employ real interpolation. Choosing $r=1 / 2-1 / 2 p$ and $s=0$ we conclude that $\mathscr{R}\left((G+A)_{21}^{-1}\right)$ is contained in

$$
{ }_{0} B_{p p}^{3 / 2-1 / 2 p}\left(J ; L_{p}\left(\mathbb{R}^{n}\right)\right) \cap{ }_{0} B_{p p}^{1-1 / 2 p}\left(J ; H_{p}^{2}\left(\mathbb{R}^{n}\right)\right) \cap_{0} B_{p p}^{1 / 2-1 / 2 p}\left(J ; H_{p}^{3}\left(\mathbb{R}^{n}\right)\right) .
$$

Thus the optimal regularity class for $\sigma$ coming from $f \in X_{1}$ is the intersection of these six spaces. This implies that one natural class of inhomogeneities to be considered is

$$
h \in{ }_{0} B_{p p}^{1 / 2-1 / 2 p}\left(J ; L_{p}\left(\mathbb{R}^{n}\right)\right) \cap L_{p}\left(J ; B_{p p}^{1-1 / p}\left(\mathbb{R}^{n}\right)\right) .
$$

To show the inclusion

$$
\mathscr{R}\left((G+A)_{21}^{-1}\right) \subset{ }_{0} H_{p}^{1}\left(J ; B_{p p}^{2-2 / p}\left(\mathbb{R}^{n}\right)\right)
$$

observe that

$$
G L^{-1}: L_{p}\left(J ; L_{p}\left(\mathbb{R}^{n}\right)\right) \rightarrow L_{p}\left(J ; L_{p}\left(\mathbb{R}^{n}\right)\right) \text { is bounded }
$$

and also that

$$
G L^{-1}:{ }_{0} H_{p}^{1 / 2}\left(J ; L_{p}\left(\mathbb{R}^{n}\right)\right) \rightarrow L_{p}\left(J ; H_{p}^{2}\left(\mathbb{R}^{n}\right)\right) \text { is bounded }
$$

hence by interpolation

$$
G L^{-1}:{ }_{0} B_{p p}^{1 / 2-1 / 2 p}\left(J ; L_{p}\left(\mathbb{R}^{n}\right)\right) \rightarrow L_{p}\left(J ; B_{p p}^{2-2 / p}\left(\mathbb{R}^{n}\right)\right) \text { is bounded. }
$$

Therefore we also have $L^{-1} \varphi \in{ }_{0} H_{p}^{1}\left(J ; B_{p p}^{2-2 / p}\left(\mathbb{R}^{n}\right)\right)$.

To deal with the entry $(G+A)_{12}^{-1}$ of $(G+A)^{-1}$, we have the representation

$$
(G+A)_{21}^{-1} h=e^{-y F} D_{n} L^{-1} h .
$$

Setting $\psi=D_{n} L^{-1} h$, this means to ask when $e^{-y F} \psi \in \mathscr{R}\left((G+A)_{11}^{-1}\right)$, or, when

$$
G e^{-y F} \psi, D_{n} e^{-y F} \psi, F^{2} e^{-y F} \psi \in X_{1}=L_{p}\left(J ; L_{p}\left(\mathbb{R}_{+}^{n+1}\right)\right)
$$


Since $F^{2}=G+D_{n}$ and $G\left(G+D_{n}\right)^{-1}, D_{n}\left(G+D_{n}\right)^{-1}$ are bounded, we have only to consider the last inclusion. But this means

$$
\int_{0}^{\infty}\left\|y^{1-(1-1 / p)} F e^{-y F} F \psi\right\|^{p} \frac{d y}{y}=\int_{0}^{\infty}\left\|F^{2} e^{-y F} \psi\right\|^{p} d y<\infty,
$$

which is equivalent to

$$
F \psi \in D_{F}(1-1 / p, p), \quad \text { and hence to } F D_{n} L^{-1} h \in D_{F}(1-1 / p, p) .
$$

By the boundedness of $F D_{n} L^{-1}$ this will be implied by $h \in D_{F}(1-1 / p, p)$, where $F$ is now considered on $L_{p}\left(J ; L_{p}\left(\mathbb{R}^{n}\right)\right)=X_{0}$. Thus

$$
h \in{ }_{0} B_{p p}^{1 / 2-1 / 2 p}\left(J ; L_{p}\left(\mathbb{R}^{n}\right)\right) \cap L_{p}\left(J ; B_{p p}^{1-1 / p}\left(\mathbb{R}^{n}\right)\right)
$$

implies that the corresponding part of $w$ belongs to the right regularity class. Finally, it is clear that $(G+A)_{22}^{-1} h=L^{-1} h$ belongs to the proper class and the proof of Proposition 3.1 is now complete.

Suppose that $h \in L_{p}\left(J ; B_{p p}^{2-2 / p}\left(\mathbb{R}^{n}\right)\right)$. Then

$$
(G+A)_{22}^{-1} h \in{ }_{0} H_{p}^{1}\left(J ; B_{p p}^{2-2 / p}\left(\mathbb{R}^{n}\right)\right) \cap{ }_{0} H_{p}^{1 / 2}\left(J ; B_{p p}^{4-2 / p}\left(\mathbb{R}^{n}\right)\right) \cap L_{p}\left(J ; B_{p p}^{5-2 / p}\left(\mathbb{R}^{n}\right)\right) .
$$

We claim that $\psi=F D_{n} L^{-1} h \in D_{F}(1-1 / p, p)$. By the boundedness of $F D_{n} L^{-1}$ we have $\psi \in L_{p}\left(J ; B_{p p}^{2-2 / p}\left(\mathbb{R}^{n}\right)\right)$. On the other hand,

$$
F D_{n} L^{-1}: L_{p}\left(J ; L_{p}\left(\mathbb{R}^{n}\right)\right) \rightarrow L_{p}\left(J ; L_{p}\left(\mathbb{R}^{n}\right)\right)
$$

is bounded and

$$
F D_{n} L^{-1}: L_{p}\left(J ; H_{p}^{2}\left(\mathbb{R}^{n}\right)\right) \rightarrow{ }_{0} H_{p}^{1 / 2}\left(J ; L_{p}\left(\mathbb{R}^{n}\right)\right)
$$

is bounded as well. By interpolation we obtain that

$$
F D_{n} L^{-1}: L_{p}\left(J ; B_{p p}^{2-2 / p}\left(\mathbb{R}^{n}\right)\right) \rightarrow{ }_{0} B_{p p}^{1 / 2-1 / 2 p}\left(J ; L_{p}\left(\mathbb{R}^{n}\right)\right)
$$

is bounded, which proves the claim. Therefore $h \in L_{p}\left(J ; B_{p p}^{2-2 / p}\left(\mathbb{R}^{n}\right)\right)$ implies

$$
(G+A)_{12}^{-1} h \in{ }_{0} H_{p}^{1}\left(J ; L_{p}\left(\mathbb{R}_{+}^{n+1}\right)\right) \cap L_{p}\left(J ; H_{p}^{2}\left(\mathbb{R}_{+}^{n+1}\right)\right),
$$

the maximal regularity class for $w$. We have proved the following result.

Corollary 3.2. Suppose $f=g=w_{0}=\sigma_{0}=0$ and $h \in L_{p}\left(J ; B_{p p}^{2-2 / p}\left(\mathbb{R}^{n}\right)\right)$. Let $(w, \sigma)$ denote the solution of (1.4). Then

$$
\begin{aligned}
& w \in{ }_{0} H_{p}^{1}\left(J ; L_{p}\left(\mathbb{R}_{+}^{n+1}\right)\right) \cap L_{p}\left(J ; H_{p}^{2}\left(\mathbb{R}_{+}^{n+1}\right)\right), \\
& \sigma \in{ }_{0} H_{p}^{1}\left(J ; B_{p p}^{2-2 / p}\left(\mathbb{R}^{n}\right)\right) \cap{ }_{0} H_{p}^{1 / 2}\left(J ; B_{p p}^{3-1 / p}\left(\mathbb{R}^{n}\right)\right) \cap L_{p}\left(J ; B_{p p}^{4-1 / p}\left(\mathbb{R}^{n}\right)\right)
\end{aligned}
$$

Moreover, $\sigma \in{ }_{0} B_{p p}^{1-1 / 2 p}\left(J ; H_{p}^{2}\left(\mathbb{R}^{n}\right)\right) \cap{ }_{0} B_{p p}^{1 / 2-1 / 2 p}\left(J ; H_{p}^{3}\left(\mathbb{R}^{n}\right)\right)$. 


\section{Inhomogeneous interface conditions}

In this section we want to obtain the corresponding optimal regularity result for

$$
\begin{cases}\partial_{t} w-\Delta_{x} w-\partial_{y}^{2} w=0, & t>0, x \in \mathbb{R}^{n}, y>0, \\ \gamma w+\Delta \sigma=g, & t>0, x \in \mathbb{R}^{n}, \\ \partial_{t} \sigma-\gamma \partial_{y} w=0, & t>0, x \in \mathbb{R}^{n}, \\ w(0, x, y)=0, & y>0, x \in \mathbb{R}^{n}, \\ \sigma(0, x)=0, & x \in \mathbb{R}^{n} .\end{cases}
$$

To determine the proper class of functions $g$ which leads to the regularity of $w$ and $\sigma$ aimed at, observe that (1.6) implies

$$
\gamma w \in B_{p p}^{1-1 / 2 p}\left(J ; L_{p}\left(\mathbb{R}^{n}\right)\right) \cap L_{p}\left(J ; B_{p p}^{2-1 / p}\left(\mathbb{R}^{n}\right)\right),
$$

see Proposition 5.1. Taking the Laplace-transform in $t$ and the Fourier transform in $x$ leads to the problem

$$
\left\{\begin{array}{l}
\lambda u+|\xi|^{2} u-\partial_{y}^{2} u=0 \\
u(0)=|\xi|^{2} \rho+\bar{g} \\
\lambda \rho-\partial_{y} u(0)=0
\end{array}\right.
$$

for $u=\hat{\tilde{\sigma}}, \rho=\hat{\tilde{\sigma}}, \bar{g}=\hat{\tilde{g}}$. The solution of the latter is easily computed to the result

$$
(u(\lambda, \xi, y), \rho(\lambda, \xi))=\left(\frac{\lambda}{\lambda+\omega|\xi|^{2}} e^{-\omega y} \bar{g}(\lambda, \xi),-\frac{\omega}{\lambda+\omega|\xi|^{2}} \bar{g}(\lambda, \xi)\right) .
$$

Hence the solution of (4.1) is given by the convolution $K * g$, where the kernel $K$ is defined via its symbol

$$
\hat{\tilde{K}}(\lambda, \xi, y)=\left(\frac{\lambda}{\lambda+|\xi|^{2} \sqrt{\lambda+|\xi|^{2}}} e^{-y \sqrt{\lambda+|\xi|^{2}}}, \frac{-\sqrt{\lambda+|\xi|^{2}}}{\lambda+|\xi|^{2} \sqrt{\lambda+|\xi|^{2}}}\right),
$$

where $y>0, \xi \in \mathbb{R}^{n}$ and $\operatorname{Re} \lambda>0$. Concerning the operator $K$, we prove the following result.

Proposition 4.1. For $1<p<\infty$, let $K$ be defined by (4.3) and let $(w, \sigma):=K * g$. Then $g \in \in_{0} B_{p p}^{1-1 / 2 p}\left(J ; L_{p}\left(\mathbb{R}^{n}\right)\right) \cap L_{p}\left(J ; B_{p p}^{2-1 / p}\left(\mathbb{R}^{n}\right)\right)$ implies that

$$
\begin{aligned}
& w \in H_{p}^{1}\left(J ; L_{p}\left(\mathbb{R}_{+}^{n+1}\right)\right) \cap L_{p}\left(J ; H_{p}^{2}\left(\mathbb{R}_{+}^{n+1}\right)\right), \\
& \sigma \in{ }_{0} B_{p p}^{3 / 2-1 / 2 p}\left(J ; L_{p}\left(\mathbb{R}^{n}\right)\right) \cap{ }_{0} B_{p p}^{1-1 / 2 p}\left(J ; H_{p}^{2}\left(\mathbb{R}^{n}\right)\right) \cap L_{p}\left(J ; B_{p p}^{4-1 / p}\left(\mathbb{R}^{n}\right)\right),
\end{aligned}
$$

as well as

$$
\sigma \in{ }_{0} H_{p}^{1}\left(J ; B_{p p}^{2-2 / p}\left(\mathbb{R}^{n}\right)\right) \cap_{0} H_{p}^{1 / 2}\left(J ; B_{p p}^{3-1 / p}\left(\mathbb{R}^{n}\right)\right) \cap_{0} B_{p p}^{1 / 2-1 / 2 p}\left(J ; H_{p}^{3}\left(\mathbb{R}^{n}\right)\right) .
$$


Proof. Using the operators introduced in Section 3 leads to the representations

$$
w=e^{-F y} G L^{-1} g, \quad \sigma=-F L^{-1} g .
$$

As in Section 3 we can conclude that $w \in{ }_{0} H_{p}^{1}\left(J ; L_{p}\left(\mathbb{R}_{+}^{n+1}\right)\right) \cap L_{p}\left(J ; H_{p}^{2}\left(\mathbb{R}_{+}^{n+1}\right)\right)$ if and only if $F G L^{-1} g \in D_{F}(1-1 / p, p)$, which means

$$
F G L^{-1} g \in{ }_{0} B_{p p}^{1 / 2-1 / 2 p}\left(J ; L_{p}\left(\mathbb{R}^{n}\right)\right) \cap L_{p}\left(J ; B_{p p}^{1-1 / p}\left(\mathbb{R}^{n}\right)\right) .
$$

Since $G L^{-1}$ is bounded, the latter is implied by

$$
g \in{ }_{0} B_{p p}^{1-1 / 2 p}\left(J ; L_{p}\left(\mathbb{R}^{n}\right)\right) \cap L_{p}\left(J ; B_{p p}^{2-1 / p}\left(\mathbb{R}^{n}\right)\right) .
$$

Observe that this is precisely the trace class for $\gamma w$ coming from optimal regularity of $w$. Next we consider $\sigma$. Since

$$
F g \in{ }_{0} B_{p p}^{1 / 2-1 / 2 p}\left(J ; L_{p}\left(\mathbb{R}^{n}\right)\right) \cap L_{p}\left(J ; B_{p p}^{1-1 / p}\left(\mathbb{R}^{n}\right)\right),
$$

the mapping properties of $L^{-1}$ imply

$$
\sigma \in{ }_{0} B_{p p}^{3 / 2-1 / 2 p}\left(J ; L_{p}\left(\mathbb{R}^{n}\right)\right) \cap_{0} B_{p p}^{1-1 / 2 p}\left(J ; H_{p}^{2}\left(\mathbb{R}^{n}\right)\right) \cap_{0} B_{p p}^{1 / 2-1 / 2 p}\left(J ; H_{p}^{3}\left(\mathbb{R}^{n}\right)\right),
$$

as well as

$$
\sigma \in{ }_{0} H_{p}^{1}\left(J ; B_{p p}^{1-1 / p}\left(\mathbb{R}^{n}\right)\right) \cap{ }_{0} H_{p}^{1 / 2}\left(J ; B_{p p}^{3-1 / p}\left(\mathbb{R}^{n}\right)\right) \cap L_{p}\left(J ; B_{p p}^{4-1 / p}\left(\mathbb{R}^{n}\right)\right) .
$$

On the other hand, as in Section 3 we obtain that

$$
G L^{-1}:{ }_{0} B_{p p}^{1 / 2-1 / 2 p}\left(J ; L_{p}\left(\mathbb{R}^{n}\right)\right) \rightarrow L_{p}\left(J ; B_{p p}^{2-2 / p}\left(\mathbb{R}^{n}\right)\right)
$$

is bounded, hence $\sigma \in{ }_{0} H_{p}^{1}\left(J ; B_{p p}^{2-2 / p}\left(\mathbb{R}^{n}\right)\right)$. This gives the optimal regularity for $\sigma$ in its natural class, thereby proving Proposition 4.1 .

\section{Traces. Proof of Theorem 1.4}

In this section we shall prove Theorem 1.4. For this purpose we need some additional results on the diffusion equation on a half space with Dirichlet conditions. Consider

$$
\left\{\begin{array}{l}
\partial_{t} u-\Delta_{x} u-\partial_{y}^{2} u=f, \quad t \in J, y>0, x \in \mathbb{R}^{n} \\
u(t, x, 0)=g(t, x) \\
u(0, x, y)=u_{0}(x, y) .
\end{array}\right.
$$

For the following result we refer to Grisvard [26], and Ladyzhenskya, Solonnikov, Ural'tseva [34], Chapter IV, §9.

Proposition 5.1. Let $p \in(1, \infty), p \neq 3 / 2, J=[0, T]$, and suppose that $u$ is the solution of $(5.1)$. Then $u \in H_{p}^{1}\left(J ; L_{p}\left(\mathbb{R}_{+}^{n+1}\right)\right) \cap L_{p}\left(J ; H_{p}^{2}\left(\mathbb{R}_{+}^{n+1}\right)\right)$ if and only if the data $\left(f, g, u_{0}\right)$ have the following properties: 
(a) $f \in L_{p}\left(J ; L_{p}\left(\mathbb{R}_{+}^{n+1}\right)\right)$,

(b) $g \in B_{p p}^{1-1 / 2 p}\left(J ; L_{p}\left(\mathbb{R}^{n}\right)\right) \cap L_{p}\left(J ; B_{p p}^{2-1 / p}\left(\mathbb{R}^{n}\right)\right)$,

(c) $u_{0} \in B_{p p}^{2-2 / p}\left(\mathbb{R}_{+}^{n+1}\right)$,

(d) $\gamma u_{0}=g(0)$ if $p>3 / 2$.

If this is the case then the traces $\gamma u_{0}$ and $g(0)$ belong to $B_{p p}^{2-3 / p}\left(\mathbb{R}^{n}\right)$ for $p>3 / 2$.

There is a companion result for the diffusion equation in a half space with Neumann conditions, that is, for the equation

$$
\left\{\begin{array}{l}
\partial_{t} u-\Delta_{x} u-\partial_{y}^{2} u=f, \quad t \in J, y>0, x \in \mathbb{R}^{n} \\
\partial_{y} u(t, x, 0)=h(t, x) \\
u(0, x, y)=u_{0}(x, y)
\end{array}\right.
$$

The corresponding result for the Neumann problem reads as follows.

Proposition 5.2. Let $p \in(1, \infty), p \neq 3, J=[0, T]$, and suppose that $u$ is the solution of (5.1). Then $u \in H_{p}^{1}\left(J ; L_{p}\left(\mathbb{R}_{+}^{n+1}\right)\right) \cap L_{p}\left(J ; H_{p}^{2}\left(\mathbb{R}_{+}^{n+1}\right)\right)$ if and only if the data $\left(f, h, u_{0}\right)$ have the following properties:

(a) $f \in L_{p}\left(J ; L_{p}\left(\mathbb{R}_{+}^{n+1}\right)\right)$,

(b) $h \in B_{p p}^{1 / 2-1 / 2 p}\left(J ; L_{p}\left(\mathbb{R}^{n}\right)\right) \cap L_{p}\left(J ; B_{p p}^{1-1 / p}\left(\mathbb{R}^{n}\right)\right)$,

(c) $u_{0} \in B_{p p}^{2-2 / p}\left(\mathbb{R}_{+}^{n+1}\right)$,

(d) $\gamma \partial_{y} u_{0}=h(0)$ if $p>3$.

If this is the case then the traces $\gamma \partial_{y} u_{0}$ and $h(0)$ belong to $B_{p p}^{1-3 / p}\left(\mathbb{R}^{n}\right)$ for $p>3$.

Before we give the proof of Theorem 1.4, a number of remarks concerning embeddings and traces of anisotropic Besov and Sobolev spaces are in order.

Remarks 5.3. (a) We have

$$
B_{p p}^{3 / 2-1 / 2 p}\left(J ; L_{p}\left(\mathbb{R}^{n}\right)\right) \cap B_{p p}^{1-1 / 2 p}\left(J ; H_{p}^{2}\left(\mathbb{R}^{n}\right)\right) \hookrightarrow H_{p}^{1}\left(J ; B_{p p}^{2-2 / p}\left(\mathbb{R}^{n}\right)\right) .
$$

In fact, by the mixed derivative theorem, see Lemma 9.7, applied to $A=(d / d t)^{1 / 2}$ and $B=D_{n}$ in $X=H_{p}^{s}\left(\mathbb{R} ; L_{p}\left(\mathbb{R}^{n}\right)\right)$, we obtain the embedding

$$
H_{p}^{s+1 / 2}\left(\mathbb{R} ; L_{p}\left(\mathbb{R}^{n}\right)\right) \cap H_{p}^{s}\left(\mathbb{R} ; H_{p}^{2}\left(\mathbb{R}^{n}\right)\right) \hookrightarrow H_{p}^{1}\left(\mathbb{R} ; H_{p}^{4 s-2}\left(\mathbb{R}^{n}\right)\right),
$$

which is valid for all $s \in(1 / 2,1)$. By interpolating these embeddings by the real method of order $(1 / 2, p)$ between $s_{1}=s+\varepsilon$ and $s_{2}=s-\varepsilon$ we obtain the desired embedding for $J=\mathbb{R}$, for $1+A+B$ is an isomorphism between 


$$
H_{p}^{s+1 / 2}\left(\mathbb{R} ; L_{p}\left(\mathbb{R}^{n}\right)\right) \cap H_{p}^{s}\left(\mathbb{R} ; H_{p}^{2}\left(\mathbb{R}^{n}\right)\right) \rightarrow H_{p}^{s}\left(\mathbb{R} ; L_{p}\left(\mathbb{R}^{n}\right)\right)
$$

as well as between

$$
B_{p p}^{s+1 / 2}\left(\mathbb{R} ; L_{p}\left(\mathbb{R}^{n}\right)\right) \cap B_{p p}^{s}\left(\mathbb{R} ; H_{p}^{2}\left(\mathbb{R}^{n}\right)\right) \rightarrow B_{p p}^{s}\left(\mathbb{R} ; L_{p}\left(\mathbb{R}^{n}\right)\right)
$$

for each $s \in \mathbb{R}$. By extension from $J$ to $\mathbb{R}$, embedding and then restriction to $J$ we get the embedding also for $J$, and setting $s=1-1 / 2 p$ yields $(5.3)$.

(b) We have

$$
B_{p p}^{1-1 / 2 p}\left(J ; H_{p}^{2}\left(\mathbb{R}^{n}\right)\right) \cap L_{p}\left(J ; B_{p p}^{4-1 / p}\left(\mathbb{R}^{n}\right)\right) \hookrightarrow H_{p}^{1 / 2}\left(J ; B_{p p}^{3-1 / p}\left(\mathbb{R}^{n}\right)\right) .
$$

The mixed derivative theorem applied to the linear operators $A=\frac{d}{d t}$ and $B=D_{n}$ in $X=L_{p}\left(\mathbb{R} ; H_{p}^{2}\left(\mathbb{R}^{n}\right)\right)$ yields

$$
H_{p}^{1}\left(\mathbb{R} ; H_{p}^{2}\left(\mathbb{R}^{n}\right)\right) \cap L_{p}\left(\mathbb{R} ; H_{p}^{4}\left(\mathbb{R}^{n}\right)\right) \hookrightarrow H_{p}^{1 / 2}\left(\mathbb{R} ; H_{p}^{3}\left(\mathbb{R}^{n}\right)\right)
$$

and trivially

$$
H_{p}^{1 / 2}\left(\mathbb{R} ; H_{p}^{2}\left(\mathbb{R}^{n}\right)\right) \cap L_{p}\left(\mathbb{R} ; H_{p}^{3}\left(\mathbb{R}^{n}\right)\right) \hookrightarrow H_{p}^{1 / 2}\left(\mathbb{R} ; H_{p}^{2}\left(\mathbb{R}^{n}\right)\right) .
$$

Interpolating these embeddings by the real interpolation method of order $(1-1 / p, p)$ and employing the reiteration theorem we get $(5.4)$ for $J=\mathbb{R}$. The case of general $J$ now follows by extension and restriction.

(c) We have

$$
B_{p p}^{1-1 / 2 p}\left(J ; H_{p}^{2}\left(\mathbb{R}^{n}\right)\right) \cap L_{p}\left(J ; B_{p p}^{4-1 / p}\left(\mathbb{R}^{n}\right)\right) \hookrightarrow B_{p p}^{1 / 2-1 / 2 p}\left(J ; H_{p}^{3}\left(\mathbb{R}^{n}\right)\right) .
$$

This follows in a similar way as in (b). By interpolating the embeddings

$$
H_{p}^{1}\left(\mathbb{R} ; H_{p}^{2}\left(\mathbb{R}^{n}\right)\right) \cap L_{p}\left(\mathbb{R} ; H_{p}^{4}\left(\mathbb{R}^{n}\right)\right) \hookrightarrow H_{p}^{1 / 2}\left(\mathbb{R} ; H_{p}^{3}\left(\mathbb{R}^{n}\right)\right),
$$

and

$$
H_{p}^{1 / 2}\left(\mathbb{R} ; H_{p}^{2}\left(\mathbb{R}^{n}\right)\right) \cap L_{p}\left(\mathbb{R} ; H_{p}^{3}\left(\mathbb{R}^{n}\right)\right) \hookrightarrow L_{p}\left(\mathbb{R} ; H_{p}^{3}\left(\mathbb{R}^{n}\right)\right)
$$

(d) Traces. For $r>0$ and $1 / p<s<2$ we have

$$
B_{p p}^{s}\left(J ; L_{p}\left(\mathbb{R}^{n}\right)\right) \cap L_{p}\left(J ; B_{p p}^{r s}\left(\mathbb{R}^{n}\right)\right) \hookrightarrow C\left(J ; B_{p p}^{r s-r / p}\left(\mathbb{R}^{n}\right)\right) .
$$

It is proved in [43] that the fundamental solution $S(t)$ of the equation $(d / d t)^{s} u+A u=f$ in a Banach space $Y$ has the following property:

$$
A S(\cdot) v \in L_{p}(J ; Y) \quad \text { iff } \quad v \in D_{A}(1-1 / p s, p) .
$$

Here $A$ is an arbitrary sectorial operator in $Y$ with spectral angle 0 , and $1 / p<s<2$. Now for $Y=L_{p}\left(\mathbb{R}^{n}\right)$ and $A=D_{n}^{r s / 2}$ we have 


$$
D_{A}(1-1 / p s, p)=B_{p p}^{r s-r / p}\left(\mathbb{R}^{n}\right) .
$$

From maximal $L_{p}$-regularity of the fractional evolution equation we may then conclude that the temporal trace operator

$$
\operatorname{tr}: H_{p}^{s}\left(J ; L_{p}\left(\mathbb{R}^{n}\right)\right) \cap L_{p}\left(J ; H_{p}^{r s}\left(\mathbb{R}^{n}\right)\right) \rightarrow B_{p p}^{r s-r / p}\left(\mathbb{R}^{n}\right)
$$

is bounded. Then we may use real interpolation to obtain the boundedness of

$$
t r: B_{p p}^{s}\left(J ; L_{p}\left(\mathbb{R}^{n}\right)\right) \cap L_{p}\left(J ; B_{p p}^{r s}\left(\mathbb{R}^{n}\right)\right) \rightarrow B_{p p}^{r s-r / p}\left(\mathbb{R}^{n}\right) .
$$

Strong continuity of the translation group yields assertion (5.6).

(e) By choosing $s=1-1 / 2 p$ and $r=2$, we obtain the boundedness of

$$
\operatorname{tr}: B_{p p}^{1-1 / 2 p}\left(J ; L_{p}\left(\mathbb{R}^{n}\right)\right) \cap L_{p}\left(J ; B_{p p}^{2-1 / p}\left(\mathbb{R}^{n}\right)\right) \rightarrow B_{p p}^{2-3 / p}\left(\mathbb{R}^{n}\right), \quad p>3 / 2 .
$$

Taking $s=1 / 2-1 / 2 p$ and $r=2$ leads to the boundedness of

$$
t r: B_{p p}^{1 / 2-1 / 2 p}\left(J ; L_{p}\left(\mathbb{R}^{n}\right)\right) \cap L_{p}\left(J ; B_{p p}^{1-1 / p}\left(\mathbb{R}^{n}\right)\right) \rightarrow B_{p p}^{1-3 / p}\left(\mathbb{R}^{n}\right), \quad p>3,
$$

and setting $s=1 / 2-1 / 2 p$ and $r=4$ yields

$$
\text { tr }: B_{p p}^{1 / 2-1 / 2 p}\left(J ; L_{p}\left(\mathbb{R}^{n}\right)\right) \cap L_{p}\left(J ; B_{p p}^{2-2 / p}\left(\mathbb{R}^{n}\right)\right) \rightarrow B_{p p}^{2-6 / p}\left(\mathbb{R}^{n}\right), \quad p>3 .
$$

In particular we derive from (a) that

$$
B_{p p}^{3 / 2-1 / 2 p}\left(J ; L_{p}\left(\mathbb{R}^{n}\right)\right) \cap B_{p p}^{1-1 / 2 p}\left(J ; H_{p}^{2}\left(\mathbb{R}^{n}\right)\right) \hookrightarrow C^{1}\left(J ; B_{p p}^{2-6 / p}\left(\mathbb{R}^{n}\right)\right), \quad p>3 .
$$

Proof of Theorem 1.4: Necessity. By Propositions 5.1 and 5.2 we obtain from $w \in H_{p}^{1}\left(J ; L_{p}\left(\mathbb{R}_{+}^{n+1}\right)\right) \cap L_{p}\left(J ; H_{p}^{2}\left(\mathbb{R}_{+}^{n+1}\right)\right)$ the properties

$$
f \in L_{p}\left(J ; L_{p}\left(\mathbb{R}_{+}^{n+1}\right)\right), \quad w_{0} \in B_{p p}^{2-2 / p}\left(\mathbb{R}_{+}^{n+1}\right),
$$

as well as

$$
\begin{gathered}
\gamma w \in B_{p p}^{1-1 / 2 p}\left(J ; L_{p}\left(\mathbb{R}^{n}\right)\right) \cap L_{p}\left(J ; B_{p p}^{2-1 / p}\left(\mathbb{R}^{n}\right)\right), \\
\gamma \partial_{y} w \in B_{p p}^{1 / 2-1 / 2 p}\left(J ; L_{p}\left(\mathbb{R}^{n}\right)\right) \cap L_{p}\left(J ; B_{p p}^{1-1 / p}\left(\mathbb{R}^{n}\right)\right) .
\end{gathered}
$$

By the regularity of $\sigma$ we have $\Delta_{x} \sigma \in B_{p p}^{1-1 / 2 p}\left(J ; L_{p}\left(\mathbb{R}^{n}\right)\right) \cap L_{p}\left(J ; B_{p p}^{2-1 / p}\left(\mathbb{R}^{n}\right)\right)$ and therefore $g$ belongs to this class. Moreover, $\partial_{t} \sigma \in B_{p p}^{1 / 2-1 / 2 p}\left(J ; L_{p}\left(\mathbb{R}^{n}\right)\right)$, and by the mixed derivative theorem, i.e. Lemma 9.7, we have $\partial_{t} \sigma \in L_{p}\left(J ; B_{p p}^{1-1 / p}\left(\mathbb{R}^{n}\right)\right)$, hence

$$
h \in B_{p p}^{1 / 2-1 / 2 p}\left(J ; L_{p}\left(\mathbb{R}^{n}\right)\right) \cap L_{p}\left(J ; B_{p p}^{1-1 / p}\left(\mathbb{R}^{n}\right)\right) .
$$

The compatibility condition in Proposition 5.1 yields $\gamma w_{0}+\Delta_{x} \sigma_{0}=g(0)$ in case $p>3 / 2$; observe that then $g(0), \gamma w_{0} \in B_{p p}^{2-3 / p}\left(\mathbb{R}^{n}\right)$. For $p>3$ we obtain from

$$
\sigma \in B_{p p}^{3 / 2-1 / 2 p}\left(J ; L_{p}\left(\mathbb{R}^{n}\right)\right) \cap B_{p p}^{1-1 / 2 p}\left(J ; H_{p}^{2}\left(\mathbb{R}^{n}\right)\right)
$$


and (5.7) that $\left(\partial_{t} \sigma\right)(0) \in B_{p p}^{2-6 / p}\left(\mathbb{R}^{n}\right)$ which by the compatibility condition in Proposition 5.2 yields $\gamma \partial_{y} w_{0}+h(0) \in B_{p p}^{2-6 / p}\left(\mathbb{R}^{n}\right)$. Observe that $2-6 / p>1-3 / p$ iff $p>3$. In case $p<3 / 2, \Delta \sigma$ does not have a trace anymore, but we have the embedding

$$
B_{p p}^{3 / 2-1 / 2 p}\left(J ; L_{p}\left(\mathbb{R}^{n}\right)\right) \cap B_{p p}^{1-1 / 2 p}\left(J ; H_{p}^{2}\left(\mathbb{R}^{n}\right)\right) \hookrightarrow C\left(J ; B_{p p}^{6-6 / p}\left(\mathbb{R}^{n}\right)\right),
$$

hence $\sigma_{0} \in B_{p p}^{6-6 / p}\left(\mathbb{R}^{n}\right)$.

\section{Proof of Theorem 1.4: Sufficiency.}

Step 1: Reductions. Suppose the data $\left(f, g, h, w_{0}, \sigma_{0}\right)$ satisfy the necessary conditions of Theorem 1.4. We restrict ourselves to the case $p>3$. Only simple modifications of the arguments given below are needed for $p<3$.

First we solve the homogeneous diffusion equation (5.1) with homogeneous Dirichlet conditions on the half-space $\mathbb{R}_{+}^{n+1}$ with initial condition $w_{0}-w_{1}$, where

$$
w_{1}:=e^{-y\left(1+D_{n}^{1 / 2}\right)} w_{00}+y e^{-y\left(1+D_{n}^{1 / 2}\right)} w_{01}
$$

with $w_{00}=\gamma w_{0}$ and $w_{01}=\left(1+D_{n}^{1 / 2}\right) w_{00}+\gamma \partial_{y} w_{0}$. Since

$$
w_{00} \in B_{p p}^{2-3 / p}\left(\mathbb{R}^{n}\right) \quad \text { and } \quad w_{01} \in B_{p p}^{1-3 / p}\left(\mathbb{R}^{n}\right)
$$

we have $w_{0}-w_{1} \in{ }_{0} B_{p p}^{2-2 / p}\left(\mathbb{R}_{+}^{n+1}\right)$, hence the corresponding solution $u$ of (5.1) belongs to the maximal regularity class for $w$. Observe that

$$
h_{1}:=\gamma \partial_{y} u \in{ }_{0} B_{p p}^{1 / 2-1 / 2 p}\left(J ; L_{p}\left(\mathbb{R}^{n}\right)\right) \cap L_{p}\left(J ; B_{p p}^{1-1 / p}\left(\mathbb{R}^{n}\right)\right),
$$

by Proposition 5.2. We may then apply Proposition 3.1 to the pair of functions $f$, $h-h_{1}-e^{-t D_{n}} h_{0}$, where $h_{0}:=h(0)$. Observe that by $h_{0} \in B_{p p}^{1-3 / p}\left(\mathbb{R}^{n}\right)$ we have

$$
e^{-t D_{n}} h_{0} \in B_{p p}^{1 / 2-1 / 2 p}\left(J ; L_{p}\left(\mathbb{R}^{n}\right)\right) \cap L_{p}\left(J ; B_{p p}^{1-1 / p}\left(\mathbb{R}^{n}\right)\right) .
$$

Therefore we may assume without loss of generality that $f=0$ and $h=e^{-t D_{n}} h_{0}$. Next we apply Proposition 4.1 to $g-e^{-t D_{n}} g_{0}$, where $g_{0}=g(0)$. Observe that by $g_{0} \in B_{p p}^{2-3 / p}\left(\mathbb{R}^{n}\right)$ we have

$$
e^{-t D_{n}} g_{0} \in B_{p p}^{1-1 / 2 p}\left(J ; L_{p}\left(\mathbb{R}^{n}\right)\right) \cap L_{p}\left(J ; B_{p p}^{2-1 / p}\left(\mathbb{R}^{n}\right)\right) \text {. }
$$

Thus we may assume that $g=e^{-t D_{n}} g_{0}$.

Thus it remains to study the following problem:

$$
\left\{\begin{array}{l}
\partial_{t} w-\Delta_{x} w-\partial_{y}^{2} w=0, \quad t \in J, y>0, x \in \mathbb{R}^{n} \\
\gamma w+\Delta_{x} \sigma=e^{-t D_{n}} g_{0} \\
\partial_{t} \sigma-\gamma \partial_{y} w=e^{-t D_{n}} h_{0} \\
w(0)=e^{-y\left(1+D_{n}^{1 / 2}\right)} w_{00}+y e^{-y\left(1+D_{n}^{1 / 2}\right)} w_{01} \\
\sigma(0)=\sigma_{0}
\end{array}\right.
$$


To prove the sufficiency part of Theorem 1.4 it is now enough to prove that the solution $\sigma$ of this problem belongs to $B_{p p}^{1-1 / 2 p}\left(J ; H_{p}^{2}\left(\mathbb{R}^{n}\right)\right) \cap L_{p}\left(J ; B_{p p}^{4-1 / p}\left(\mathbb{R}^{n}\right)\right)$, since by Proposition 5.1 the equation for $\sigma$ yields $w \in H_{p}^{1}\left(J ; L_{p}\left(\mathbb{R}_{+}^{n+1}\right)\right) \cap L_{p}\left(J ; H_{p}^{2}\left(\mathbb{R}_{+}^{n+1}\right)\right)$ and $\sigma \in B_{p p}^{3 / 2-1 / 2 p}\left(J ; L_{p}\left(\mathbb{R}^{n}\right)\right)$.

Step 2: Representation of $\sigma$. We set $g_{1}=e^{-t D_{n}} g_{0}, h_{1}=e^{-t D_{n}} h_{0}$ and we let $\sigma_{0}, w_{1}, w_{00}, w_{01}$ be as in Step 1. Taking the Laplace transform w.r.t. $t$ and the Fourier transform w.r.t. $x$ leads to the following ODE-problem for $u=\hat{\tilde{w}}$ and $\rho=\hat{\tilde{\sigma}}$ :

$$
\left\{\begin{array}{l}
\omega^{2} u-\partial_{y}^{2} u=e^{-(1+|\xi|) y} u_{00}+y e^{-(1+|\xi|) y} u_{01}, \quad y>0 \\
u(0)=|\xi|^{2} \rho+\tilde{g}_{0} / \omega^{2} \\
\lambda \rho-\partial_{y} u(0)=\rho_{0}+\tilde{h}_{0} / \omega^{2}
\end{array}\right.
$$

where $u_{0 j}=\tilde{w}_{0 j}, j=0,1$. A straightforward calculation yields the following representation for $\rho$ :

$$
\begin{aligned}
\rho & =\frac{1}{\lambda+|\xi|^{2} \omega}\left[\rho_{0}-\frac{\tilde{g}_{0}}{\omega}+\frac{\tilde{h}_{0}}{\omega^{2}}+\int_{0}^{\infty} e^{-[\omega+1+|\xi|] s}\left[u_{00}+s u_{01}\right] d s\right] \\
& =\frac{1}{\lambda+|\xi|^{2} \omega}\left[\rho_{0}-\frac{\tilde{g}_{0}}{\omega}+\frac{\tilde{h}_{0}}{\omega^{2}}+\frac{u_{00}}{\omega+1+|\xi|}+\frac{u_{01}}{[\omega+1+|\xi|]^{2}}\right] .
\end{aligned}
$$

Employing the compatibility condition

$$
u_{00}=\gamma \tilde{w}_{0}=|\xi|^{2} \rho_{0}+\tilde{g}_{0}
$$

as well as

$$
u_{01}=(1+|\xi|) u_{00}+\gamma \partial_{y} \tilde{w}_{0}
$$

and setting

$$
\rho_{1}:=\tilde{\sigma}_{1}:=\tilde{h}_{0}+\gamma \partial_{y} \tilde{w}_{0}
$$

a simple computation yields

$$
\begin{aligned}
\rho= & \frac{1}{\lambda+|\xi|^{2} \omega}\left[1+\frac{|\xi|^{2}}{\omega+1+|\xi|}+\frac{|\xi|^{2}(1+|\xi|)}{[\omega+1+|\xi|]^{2}}\right] \rho_{0} \\
& +\frac{1}{\lambda+|\xi|^{2} \omega}\left[\frac{1}{\omega+1+|\xi|}+\frac{1+|\xi|}{[\omega+1+|\xi|]^{2}}-\frac{1}{\omega}\right] \tilde{g}_{0} \\
& +\frac{1}{\lambda+|\xi|^{2} \omega}\left[\frac{1}{\omega^{2}}-\frac{1}{[\omega+1+|\xi|]^{2}}\right] \tilde{h}_{0} \\
& +\frac{1}{\lambda+|\xi|^{2} \omega} \cdot \frac{1}{[\omega+1+|\xi|]^{2}} \rho_{1} \\
= & m_{1} \rho_{0}+m_{2} \tilde{g}_{0}+m_{3} \tilde{h}_{0}+m_{4} \rho_{1} .
\end{aligned}
$$


We write the coefficients $m_{j}$ in the following form:

$$
\begin{aligned}
& m_{1}=\frac{1}{\omega^{2}}+\left[\frac{\omega^{2}}{\lambda+|\xi|^{2} \omega}-\frac{\omega^{2}}{(\omega+1+|\xi|)^{2}} \cdot \frac{\omega(1+|\xi|)^{2}}{\left(\lambda+|\xi|^{2} \omega\right)}\right] \frac{|\xi|^{2}}{\omega^{4}}, \\
& m_{2}=-\left[\frac{\omega}{\omega+1+|\xi|}\right]^{2} \cdot \frac{\omega(1+|\xi|)^{2}}{\lambda+\omega|\xi|^{2}} \cdot \frac{1}{\omega^{4}}, \\
& m_{3}=\left[\frac{\omega}{\omega+1+|\xi|}\right]^{2} \cdot \frac{2 \omega(1+|\xi|)^{2}+(1+|\xi|)^{3}}{\lambda+\omega|\xi|^{2}} \cdot \frac{1}{\omega^{4}} \cdot \frac{1}{1+|\xi|}, \\
& m_{4}=\frac{1}{\lambda+|\xi|^{2} \omega} \cdot \frac{1}{\left[\omega+1+\left.|\xi|\right|^{2}\right.} .
\end{aligned}
$$

Step 3: Regularity. We shall now deal with each of the four terms in (5.11) separately. By taking inverse transforms we obtain for the second one

$$
w_{2}:=-\left[F\left(F+1+D_{n}^{1 / 2}\right)^{-1}\right]^{2} \cdot F\left(1+D_{n}^{1 / 2}\right)^{2} L^{-1} F^{-2} w_{20},
$$

where $w_{20}:=e^{-t D_{n}} g_{0}$. Now, $g_{0} \in B_{p p}^{2-3 / p}\left(\mathbb{R}^{n}\right)$ implies $w_{20} \in L_{p}\left(J ; B_{p p}^{2-1 / p}\left(\mathbb{R}^{n}\right)\right)$. By the properties of $F^{-1}$ this yields

$$
F^{-2} w_{20} \in{ }_{0} H_{p}^{1}\left(J ; B_{p p}^{2-1 / p}\left(\mathbb{R}^{n}\right)\right) \cap L_{p}\left(J ; B_{p p}^{4-1 / p}\left(\mathbb{R}^{n}\right)\right),
$$

hence by boundedness of $F\left(F+1+D_{n}^{1 / 2}\right)^{-1}$ and $F\left(1+D_{n}^{1 / 2}\right)^{2} L^{-1}$ we have

$$
w_{2} \in{ }_{0} H_{p}^{1}\left(J ; B_{p p}^{2-1 / p}\left(\mathbb{R}^{n}\right)\right) \cap L_{p}\left(J ; B_{p p}^{4-1 / p}\left(\mathbb{R}^{n}\right)\right) .
$$

Therefore $w_{2}$ enjoys the right regularity properties, as the embedding

$$
{ }_{0} H_{p}^{1}\left(J ; B_{p p}^{2-1 / p}\left(\mathbb{R}^{n}\right)\right) \cap L_{p}\left(J ; B_{p p}^{4-1 / p}\left(\mathbb{R}^{n}\right)\right) \hookrightarrow{ }_{0} B_{p p}^{1-1 / 2 p}\left(J ; H_{p}^{2}\left(\mathbb{R}^{n}\right)\right)
$$

shows.

Similarly, for the third term we obtain

$$
w_{3}:=\left[F\left(F+1+D_{n}^{1 / 2}\right)^{-1}\right]^{2} \cdot\left[2 F\left(1+D_{n}^{1 / 2}\right)^{2}+\left(1+D_{n}^{1 / 2}\right)^{3}\right] L^{-1} F^{-2} w_{30},
$$

with $\quad w_{30}:=e^{-D_{n} t}\left(1+D_{n}^{1 / 2}\right)^{-1} h_{0}$. From $h_{0} \in B_{p p}^{1-3 / p}\left(\mathbb{R}^{n}\right) \quad$ we can conclude that $w_{30} \in L_{p}\left(J ; B_{p p}^{2-1 / p}\left(\mathbb{R}^{n}\right)\right)$, and so

$$
F^{-2} w_{30} \in{ }_{0} H_{p}^{1}\left(J ; B_{p p}^{2-1 / p}\left(\mathbb{R}^{n}\right)\right) \cap L_{p}\left(J ; B_{p p}^{4-1 / p}\left(\mathbb{R}^{n}\right)\right) .
$$

The terms $F\left(F+1+D_{n}^{1 / 2}\right)^{-1}$ and $\left[2 F\left(1+D_{n}^{1 / 2}\right)^{2}+\left(1+D_{n}^{1 / 2}\right)^{3}\right] L^{-1}$ are bounded, hence

$$
w_{3} \in{ }_{0} H_{p}^{1}\left(J ; B_{p p}^{2-1 / p}\left(\mathbb{R}^{n}\right)\right) \cap L_{p}\left(J ; B_{p p}^{4-1 / p}\left(\mathbb{R}^{n}\right)\right),
$$

that is, $w_{3}$ has the desired regularity. 
We write the first term in the following way.

$$
w_{1}:=w_{10}+\left[F^{2} L^{-1}-\left[F\left(F+1+D_{n}^{1 / 2}\right)^{-1}\right]^{2} F\left(1+D_{n}^{1 / 2}\right)^{2} L^{-1}\right] F^{-2} D_{n} w_{10}
$$

with $w_{10}:=e^{-t D_{n}} \sigma_{0}$. Since $\sigma_{0} \in B_{p p}^{4-3 / p}\left(\mathbb{R}^{n}\right)$ we conclude that

$$
w_{10} \in H_{p}^{1}\left(J ; B_{p p}^{2-1 / p}\left(\mathbb{R}^{n}\right)\right) \cap L_{p}\left(J ; B_{p p}^{4-1 / p}\left(\mathbb{R}^{n}\right)\right)
$$

and also that $F^{-2} D_{n} w_{10} \in{ }_{0} H_{p}^{1}\left(J ; B_{p p}^{2-1 / p}\left(\mathbb{R}^{n}\right)\right) \cap L_{p}\left(J ; B^{4-1 / p}\left(\mathbb{R}^{n}\right)\right)$. Hence the boundedness of $F^{2} L^{-1}, F\left(F+1+D_{n}^{1 / 2}\right)^{-1}$, and of $F\left(1+D_{n}^{1 / 2}\right)^{2} L^{-1}$ yields the regularity assertion for $w_{1}$. Moreover, we have that $w_{1}(0)=\sigma_{0}$.

We now consider the last term

$$
w_{4}:=\left[F\left(F+1+D_{n}^{1 / 2}\right)^{-1}\right]^{2} L^{-1} w_{40},
$$

with $w_{40}:=e^{-t D_{n}} \sigma_{1}$. Then $\sigma_{1} \in B_{p p}^{2-6 / p}\left(\mathbb{R}^{n}\right)$ implies $w_{40} \in L_{p}\left(J ; B_{p p}^{2-4 / p}\left(\mathbb{R}^{n}\right)\right)$, hence

$$
L^{-1} w_{40} \in{ }_{0} H_{p}^{1}\left(J ; B_{p p}^{2-4 / p}\left(\mathbb{R}^{n}\right)\right) \cap{ }_{0} H_{p}^{1 / 2}\left(J ; B_{p p}^{4-4 / p}\left(\mathbb{R}^{n}\right)\right) \cap L_{p}\left(J ; B_{p p}^{5-4 / p}\left(\mathbb{R}^{n}\right)\right) .
$$

Since $5-4 / p>4-1 / p$ in case $p>3$, we obtain from this and the boundedness of $F\left(F+1+D_{n}^{1 / 2}\right)^{-1}$ the regularity $w_{4} \in L_{p}\left(J ; B_{p p}^{4-1 / p}\left(\mathbb{R}^{n}\right)\right)$. However, in this way it is not possible to show $w_{4} \in B_{p p}^{1-1 / 2 p}\left(J ; H_{p}^{2}\left(\mathbb{R}^{n}\right)\right)$ and a different argument is needed. It is given in Step 4 below. But we may restrict our attention to the regularity $D_{n} w_{4} \in B_{p p}^{1-1 / 2 p}\left(J ; L_{p}\left(\mathbb{R}^{n}\right)\right)$ since we already know that $w_{4} \in H_{p}^{1}\left(J ; B_{p p}^{2-4 / p}\left(\mathbb{R}^{n}\right)\right)$ which is embedded into $B_{p p}^{1-1 / 2 p}\left(J ; L_{p}\left(\mathbb{R}^{n}\right)\right)$. We now set $\sigma_{2}:=\left(1+D_{n}\right)^{-1} \sigma_{1}$ and we observe that $\sigma_{2} \in B_{p p}^{4-6 / p}\left(\mathbb{R}^{n}\right)$ and that $\sigma_{1}=D_{n} \sigma_{2}+\sigma_{2}$. The contribution to $w_{4}$ coming from $\sigma_{2}$ then belongs to ${ }_{0} H_{p}^{1}\left(J ; B_{p p}^{4-4 / p}\left(\mathbb{R}^{n}\right)\right)$ which is embedded in ${ }_{0} H_{p}^{1}\left(J ; B_{p p}^{2-1 / p}\left(\mathbb{R}^{n}\right)\right)$. We may therefore assume that

$$
\sigma_{1}=D_{n} \sigma_{2} \quad \text { with } \sigma_{2} \in B_{p p}^{4-6 / p}\left(\mathbb{R}^{n}\right)
$$

Step 4. We first invert the Laplace transform in the following way. Choose a contour $\Gamma=e^{-i \theta}[\infty, 0] \cup e^{i \theta}[0, \infty]$ with $\pi>\theta>\pi / 2$ fixed. Then we obtain

$$
\tilde{w}_{4}(t, \xi)=\frac{1}{2 \pi i} \int_{\Gamma} e^{\lambda t} m_{4}(\lambda, \xi) \rho_{1}(\xi) d \lambda, \quad t>0, \xi \in \mathbb{R}^{n}
$$

and this integral is absolutely convergent for each $t>0$ and $\xi \in \mathbb{R}^{n}$. Then we may change variables $\lambda=|\xi|^{4} z$ to the result

$$
\tilde{w}_{4}(t, \xi)=\frac{1}{2 \pi i} \int_{\Gamma} e^{t z|\xi|^{4}} \frac{\rho_{1}(\xi) d z}{\left[z+\left(z+|\xi|^{-2}\right)^{1 / 2}\right]\left[1+|\xi|+|\xi|^{2}\left(z+|\xi|^{-2}\right)^{1 / 2}\right]^{2}}
$$

Using the functional calculus for $D_{n}$ in $L_{p}\left(\mathbb{R}^{n}\right)$ and inverting the Fourier transform, we get the following representation for $w_{4}$, where we use the assumption $\sigma_{1}=D_{n} \sigma_{2}$ with $\sigma_{2} \in B_{p p}^{4-6 / p}\left(\mathbb{R}^{n}\right)$ : 


$$
w_{4}(t, \cdot)=\frac{1}{2 \pi i} \int_{\Gamma} e^{t z D_{n}^{2}}\left(z+\left(z+D_{n}^{-1}\right)^{1 / 2}\right)^{-1} D_{n}\left(1+D_{n}^{1 / 2}+D_{n}\left(z+D_{n}^{-1}\right)^{1 / 2}\right)^{-2} \sigma_{2}(\cdot) d z .
$$

Observe that the estimates

$$
\left\|\left(z+\left(z+D_{n}^{-1}\right)^{1 / 2}\right)^{-1}\right\|_{L_{p}\left(\mathbb{R}^{n}\right)} \leqq C_{p} \frac{1}{\sqrt{|z|}+|z|}
$$

as well as

$$
\left\|D_{n}^{2}\left(1+D_{n}^{1 / 2}+D_{n}\left(z+D_{n}^{-1}\right)^{1 / 2}\right)^{-2}\right\|_{L_{p}\left(\mathbb{R}^{n}\right)} \leqq C_{p} /|z|
$$

and

$$
\left\|D_{n}\left(1+D_{n}^{1 / 2}+D_{n}\left(z+D_{n}^{-1}\right)^{1 / 2}\right)^{-2}\right\|_{L_{p}\left(\mathbb{R}^{n}\right)} \leqq C_{p}
$$

hold on $\Gamma$ for some constant $C_{p}$, depending only on $p$. This shows that the integral defining $w_{4}$ is absolutely convergent in $L_{p}\left(\mathbb{R}^{n}\right)$ for each fixed $t>0$. Next observe that $B_{p p}^{4-6 / p}\left(\mathbb{R}^{n}\right)=D_{D_{n}^{2}}(1-3 / 2 p, p)$ in $L_{p}\left(\mathbb{R}^{n}\right)$, and that we only have to show $t^{1 / 2 p} D_{n} \dot{w}_{4} \in L_{p}\left(J ; L_{p}\left(\mathbb{R}^{n}\right)\right)$, by Hardy's inequality. Now we have by the representation formula and the above estimates

$$
\begin{aligned}
& \left\|t^{1 / 2 p} D_{n} \dot{w}_{4}\right\|_{L_{p}\left(J ; L_{p}\left(\mathbb{R}^{n}\right)\right)} \\
& \leqq C \int_{\Gamma} \| t^{1 / 2 p} D_{n}^{2} e^{t z D_{n}^{2}}\left[\left(z+\left(z+D_{n}^{-1}\right)^{1 / 2}\right)^{-1}\right] \cdot \\
& \quad z D_{n}^{2}\left(1+D_{n}^{1 / 2}+D_{n}\left(z+D_{n}^{-1}\right)^{1 / 2}\right)^{-2} \sigma_{2} \|_{L_{p}\left(J ; L_{p}\left(\mathbb{R}^{n}\right)\right)}|d z| \\
& \leqq C \int_{\Gamma}\left\|t^{1 / 2 p} D_{n}^{2} e^{t z D_{n}^{2}} \sigma_{2}\right\|_{L_{p}\left(J ; L_{p}\left(\mathbb{R}^{n}\right)\right)} \frac{|d z|}{|z|+\sqrt{|z|}} \\
& \leqq C \int_{\Gamma}|z|^{-3 / 2 p} \frac{|d z|}{|z|+\sqrt{|z|}}\left\|\sigma_{2}\right\|_{B_{p p}^{4-6 / p}\left(\mathbb{R}^{n}\right)} \\
& =C\left\|\sigma_{2}\right\|_{B_{p p}^{4-6 / p}\left(\mathbb{R}^{n}\right)},
\end{aligned}
$$

which proves the result.

Proof of Theorem 1.5. The assertions of Theorem 1.5 follow along the lines of the above proof, using in addition Corollary 3.2.

\section{The two-phase problem}

In this section we consider the linear two-phase problem on $\mathbb{R}^{n} \times \mathbb{R}$, where $\dot{\mathbb{R}}=\mathbb{R} \backslash\{0\}$, that is, we consider 


$$
\begin{cases}\partial_{t} w-\Delta_{x} w-\partial_{y}^{2} w=f, & t>0, x \in \mathbb{R}^{n}, y \in \dot{\mathbb{R}} \\ \gamma w^{ \pm}+\Delta \sigma=g, & t>0, x \in \mathbb{R}^{n}, \\ \partial_{t} \sigma-\left[\partial_{y} w\right]=h, & t>0, x \in \mathbb{R}^{n}, \\ w(0, x, y)=w_{0}(x, y), & y \in \mathbb{R}, x \in \mathbb{R}^{n}, \\ \sigma(0, x)=\sigma_{0}(x), & x \in \mathbb{R}^{n},\end{cases}
$$

where the initial values $\left(w_{0}, \sigma_{0}\right)$ and the inhomogeneities $(f, g, h)$ are given. Moreover, $\left[\partial_{y} w\right]:=\left(\left.\partial_{y} w\right|_{y=0^{+}}-\left.\partial_{y} w\right|_{y=0^{-}}\right)$denotes the jump of the normal derivatives of $w$ across $y=0$. As before, taking the Laplace transform in $t$ and the Fourier transform in $x$ leads to the problem

$$
\left\{\begin{array}{l}
\left(\lambda+|\xi|^{2}\right) u-\partial_{y}^{2} u=\bar{f} \\
u(0)=|\xi|^{2} \rho+\bar{g} \\
\lambda \rho-\left[\partial_{y} u\right](0)=\bar{h}
\end{array}\right.
$$

where $u=\hat{\tilde{w}}, \rho=\hat{\tilde{\sigma}}, \bar{f}=\hat{\tilde{f}}+\tilde{w}_{0}, \bar{g}=\hat{\tilde{g}}$, and $\bar{h}=\hat{\tilde{h}}+\tilde{\sigma}_{0}$. It is easy to compute $u$ and $\rho$ to the result

$$
\rho=\frac{1}{\lambda+2 \omega|\xi|^{2}}\left[\bar{h}-2 \omega \bar{g}+\int_{\mathbb{R}} e^{-\omega|s|} \bar{f}(s) d s\right],
$$

and

$$
\begin{aligned}
u= & \int_{\mathbb{R}} \frac{e^{-\omega|y-s|}}{2 \omega} \bar{f}(s) d s-\frac{\lambda}{\lambda+2 \omega|\xi|^{2}} \int_{\mathbb{R}} \frac{e^{-\omega(|y|+|s|)}}{2 \omega} \bar{f}(s) d s \\
& +\frac{\lambda}{\lambda+2 \omega|\xi|^{2}} e^{-\omega|y|} \bar{g}+\frac{|\xi|^{2}}{\lambda+2 \omega|\xi|^{2}} e^{-\omega|y|} \bar{h} .
\end{aligned}
$$

This representation of the solution $(w, \sigma)$ of $(6.1)$ involves the same ingredients as for the solution of (1.4), with some minor modifications. This shows that the results for the onephase problem carry over to the two-phase problem in a straightforward manner. Here we only state the result corresponding to Theorem 1.4.

Theorem 6.1. Assume that $1<p<\infty$ with $p \neq 3 / 2,3$ and let $J=[0, T]$. Then there exists a unique solution $(w, \sigma)$ to problem $(6.1)$ with

$$
\begin{aligned}
& w \in H_{p}^{1}\left(J ; L_{p}\left(\mathbb{R}^{n} \times \dot{\mathbb{R}}\right)\right) \cap L_{p}\left(J ; H_{p}^{2}\left(\mathbb{R}^{n} \times \dot{\mathbb{R}}\right)\right), \\
& \sigma \in B_{p p}^{3 / 2-1 / 2 p}\left(J ; L_{p}\left(\mathbb{R}^{n}\right)\right) \cap B_{p p}^{1-1 / 2 p}\left(J ; H_{p}^{2}\left(\mathbb{R}^{n}\right)\right) \cap L_{p}\left(J ; B_{p p}^{4-1 / p}\left(\mathbb{R}^{n}\right)\right),
\end{aligned}
$$

if and only if the data $\left(f, g, h, w_{0}, \sigma_{0}\right)$ satisfy the following conditions:

(a) $f \in L_{p}\left(J ; L_{p}\left(\mathbb{R}^{n+1}\right)\right)$ and $w_{0} \in B_{p p}^{2-2 / p}\left(\mathbb{R}^{n} \times \dot{\mathbb{R}}\right)$,

(b) $g \in B_{p p}^{1-1 / 2 p}\left(J ; L_{p}\left(\mathbb{R}^{n}\right)\right) \cap L_{p}\left(J ; B_{p p}^{2-1 / p}\left(\mathbb{R}^{n}\right)\right)$, 
(c) $h \in B_{p p}^{1 / 2-1 / 2 p}\left(J ; L_{p}\left(\mathbb{R}^{n}\right)\right) \cap L_{p}\left(J ; B_{p p}^{1-1 / p}\left(\mathbb{R}^{n}\right)\right)$,

(d) $\sigma_{0} \in B_{p p}^{6-6 / p}\left(\mathbb{R}^{n}\right)$ for $p<3 / 2$, and $\sigma_{0} \in B_{p p}^{4-3 / p}\left(\mathbb{R}^{n}\right)$ for $p>3 / 2$,

(e) $\gamma w_{0}+\Delta \sigma_{0}=g(0)$ in case $p>3 / 2$,

(f) $\left[\partial_{y} w_{0}\right]+h(0) \in B_{p p}^{2-6 / p}\left(\mathbb{R}^{n}\right)$ in case $p>3$.

\section{Existence and uniqueness for the nonlinear problem}

Based on the results of the preceding sections we now investigate the nonlinear problem (1.3). We first transform system (1.3) to a problem on a fixed domain. For this we fix $a>0$ and choose $\varphi \in C^{\infty}(\mathbb{R})$ with $\varphi(\mathbb{R}) \subset[0,1]$ and

$$
\varphi(y)=\left\{\begin{array}{ll}
1, & |y| \leqq a \\
0, & |y|>3 a,
\end{array} \quad\left\|\varphi^{\prime}\right\|_{L_{\infty}}<\frac{1}{a} .\right.
$$

Moreover, we fix $p>n+3$. By Sobolev's embedding theorem we have

$$
B_{p p}^{4-3 / p}\left(\mathbb{R}^{n}\right) \hookrightarrow B U C^{3}\left(\mathbb{R}^{n}\right) .
$$

Given $\rho \in B_{p p}^{4-3 / p}\left(\mathbb{R}^{n}\right)$, define

$$
\theta_{\rho}(x, y):=(x, y+\varphi(y) \rho(x)), \quad(x, y) \in \mathbb{R}^{n} \times \mathbb{R} \equiv \mathbb{R}^{n+1} .
$$

We write $\mathbb{R}_{+}^{n+1}:=\mathbb{R}^{n} \times(0, \infty), \mathbb{R}_{-}^{n+1}:=\mathbb{R}^{n} \times(-\infty, 0)$, and $\dot{\mathbb{R}}:=\mathbb{R} \backslash\{0\}$. Furthermore, we let

$$
\Omega_{\rho}:=\theta_{\rho}\left(\mathbb{R}^{n} \times \dot{\mathbb{R}}\right), \quad \Omega_{\rho}^{ \pm}:=\theta_{\rho}\left(\mathbb{R}_{ \pm}^{n+1}\right), \quad \Gamma_{\rho}:=\theta_{\rho}\left(\mathbb{R}^{n} \times\{0\}\right)=\operatorname{graph}(\rho) .
$$

Identifying $\mathbb{R}^{n} \times\{0\}$ and $\mathbb{R}^{n}$, we clearly have

$$
\theta_{\rho} \in \operatorname{Diff}^{3}\left(\mathbb{R}^{n}, \Gamma_{\rho}\right)
$$

We also need the following result.

Lemma 7.1. Given $\rho \in B_{p p}^{4-3 / p}\left(\mathbb{R}^{n}\right)$ satisfying $\|\rho\|_{\infty}<a$, we have

$$
\theta_{\rho} \in \operatorname{Diff}^{3}\left(\mathbb{R}^{n} \times \dot{\mathbb{R}}, \Omega_{\rho}\right) \cap \operatorname{Diff}^{3}\left(\mathbb{R}_{ \pm}^{n+1}, \Omega_{\rho}^{ \pm}\right) .
$$

Proof. This follows from the fact that $[y \mapsto y+\rho(x) \varphi(y)]$ is a smooth strictly increasing diffeomorphism from $\mathbb{R}$ to $\mathbb{R}$.

The diffeomorphism $\theta_{\rho}$ is a particular case of the so-called Hanzawa transformation [29]. Of course, there is a great liberty in choosing transformations from $\mathbb{R}_{ \pm}^{n+1}$ onto $\Omega_{\rho}^{ \pm}$. A technically very easy situation is obtained by considering

$$
(x, y) \mapsto(x, y+\rho(x)), \quad(x, y) \in \mathbb{R}^{n+1} .
$$


Observe that $\theta_{\rho}$ leaves the points outside strip containing $\Gamma_{\rho}$ unchanged, whereas (7.2) acts in general as a true transformation on the whole of $\mathbb{R}^{n+1}$.

Given any function $u$ defined on $\Omega_{\rho}$, we use the notation $u^{ \pm}:=u \mid \Omega_{\rho}^{ \pm}$. A similar convention is used for functions on $\mathbb{R}^{n} \times \dot{\mathbb{R}}$.

It follows from Lemma 7.1, equation (7.1), and the mean value theorem that

$$
\theta_{\rho}^{*} \in \operatorname{Isom}\left(H_{p}^{k}\left(\Omega_{\rho}\right), H_{p}^{k}\left(\mathbb{R}^{n} \times \dot{\mathbb{R}}\right)\right), \quad k=0,1,2,
$$

with

$$
\left[\theta_{\rho}^{*}\right]^{-1}=\theta_{*}^{\rho}
$$

where

$$
\theta_{\rho}^{*} u:=u \circ \theta_{\rho}, \quad u \in L_{p}\left(\Omega_{\rho}\right) \quad \text { and } \quad \theta_{*}^{\rho} v:=v \circ \theta_{\rho}^{-1}, \quad v \in L_{p}\left(\mathbb{R}^{n} \times \dot{\mathbb{R}}\right)
$$

denote the pull-back and push-forward operators induced by $\theta_{\rho}$. Moreover, if $\rho \in B U C^{\infty}\left(\mathbb{R}^{n}\right)$ then

$$
\theta_{\rho}^{*} \in \operatorname{Isom}\left(B U C^{\infty}\left(\Omega_{\rho}^{ \pm}\right), B U C^{\infty}\left(\mathbb{R}_{ \pm}^{n+1}\right)\right)
$$

Observe that, given $v: \mathbb{R}^{n} \times \dot{\mathbb{R}} \rightarrow \mathbb{R}$ such that $v^{ \pm} \in B U C^{\infty}\left(\mathbb{R}_{ \pm}^{n+1}\right)$, there exist unique extensions $\bar{v}^{+}$of $v^{+}$and $\bar{v}^{-}$of $v^{-}$to $\mathbb{R}^{n} \times[0, \infty)$ and to $\mathbb{R}^{n} \times(-\infty, 0]$, respectively. However, in general, $\partial_{j}^{k} \bar{v}^{+}(x, 0) \neq \partial_{j}^{k} \bar{v}^{-}(x, 0)$ for all $x \in \mathbb{R}^{n}, k \in \mathbb{N}, j=1, \ldots, n+1$. In particular, $v$ does in general not belong to $B U C^{\infty}\left(\mathbb{R}^{n+1}\right)$. In addition, $\mathbb{R}^{n} \times \mathbb{R}$ clearly does not satisfy the segment property, cf. Section 3.17 in [1], and the Sobolev embedding theorem does not hold for $\mathbb{R}^{n} \times \mathbb{R}$. Of course, the Sobolev embedding theorem holds true for the domains $\mathbb{R}_{ \pm}^{n+1}$.

Fix $T>0$ and assume that $\rho:[0, T] \rightarrow B_{p p}^{4-3 / p}\left(\mathbb{R}^{n}\right)$ satisfies $\|\rho(t)\|_{L_{\infty}}<a$. Set

$$
X(t, x):=(x, \rho(t, x)), \quad(t, x) \in[0, T] \times \mathbb{R}^{n} .
$$

Clearly, given $t \in[0, T]$, the function $X(t, \cdot)$ is a parametrization of $\Gamma_{\rho(t)}$ with

$$
v(t, X(t, x))=\frac{(-\nabla \rho(t, x), 1)}{\sqrt{1+|\nabla \rho(t, x)|^{2}}} .
$$

If $X$ is differentiable at $\left(t_{0}, x_{0}\right) \in(0, T) \times \mathbb{R}^{n}$, we conclude that

$$
V\left(t_{0}, X\left(t_{0}, x_{0}\right)\right)=\left(\partial_{t} X\left(t_{0}, x_{0}\right) \mid v\left(t_{0}, X\left(t_{0}, x_{0}\right)\right)\right)=\frac{\partial_{t} \rho\left(t_{0}, x_{0}\right)}{\sqrt{1+\left|\nabla \rho\left(t_{0}, x_{0}\right)\right|^{2}}} .
$$

Hence the Stefan condition $V=\left[\partial_{\nu} u\right]$ becomes

$$
\partial_{t} \rho-\sqrt{1+|\nabla \rho|^{2}} \theta_{\rho}^{*}\left[\partial_{\nu} u\right]=0 \quad \text { on } \mathbb{R}^{n}, t>0 .
$$


Furthermore, it is well-known that

$$
\kappa(X(t, x))=-\operatorname{div}\left(\frac{\nabla \rho(t, x)}{\sqrt{1+|\nabla \rho(t, x)|^{2}}}\right), \quad(t, x) \in[0, T] \times \mathbb{R}^{n},
$$

since $\Gamma_{\rho(t)}$ is the graph of $\rho(t)$ over $\mathbb{R}^{n}$, cf. (A.14) in [25]. Consequently, system (1.3) becomes

$$
\begin{cases}\partial_{t} u-\Delta u=0 & \text { in } \Omega_{\rho(t)}, t>0, \\ \theta_{\rho}^{*} u^{ \pm}+\operatorname{div}\left(\frac{\nabla \rho}{\sqrt{1+|\nabla \rho|^{2}}}\right)=0 & \text { on } \mathbb{R}^{n}, t \geqq 0 \\ \partial_{t} \rho-\sqrt{1+|\nabla \rho|^{2}} \theta_{\rho}^{*}\left[\partial_{v} u\right]=0 & \text { on } \mathbb{R}^{n}, t>0 \\ \rho(0, \cdot)=\rho_{0} & \text { on } \mathbb{R}^{n}, \\ u(0, \cdot)=u_{0} & \text { in } \Omega_{\rho_{0}}^{ \pm}\end{cases}
$$

where $\rho_{0}: \mathbb{R}^{n} \rightarrow \mathbb{R}$ describes the initial position of $\Gamma$ and $u_{0}: \Omega_{\rho_{0}} \rightarrow \mathbb{R}$ stands for the initial temperature distribution.

To give a precise notion of solutions to (7.5), we shall transform the differential operators acting on $u$. Given $\rho \in B_{p p}^{4-3 / p}\left(\mathbb{R}^{n}\right)$, let

$$
\begin{aligned}
\mathscr{A}(\rho) v & :=-\theta_{\rho}^{*}\left(\Delta\left(\theta_{*}^{\rho} v\right)\right), \\
{[\mathscr{B}(\rho) v]: } & =-\sqrt{1+|\nabla \rho|^{2}} \theta_{\rho}^{*}\left(\gamma\left(\nabla\left(\theta_{*}^{\rho}\left(v^{+}-v^{-}\right)\right)\right) \mid v\right),
\end{aligned}
$$

for $v \in H_{p}^{2}\left(\mathbb{R}^{n} \times \dot{\mathbb{R}}\right)$. Here $\Delta$ and $\nabla$ stand for the Laplace and the gradient operator on $\mathbb{R}^{n+1}$, respectively, and $\gamma$ is the trace operator with respect to $\Gamma_{\rho}$. Finally, let

$$
m(v, \rho)(x, y):=-\frac{\varphi(y)}{1+\varphi^{\prime}(y) \rho(x)}[\mathscr{B}(\rho) v](x), \quad(x, y) \in \mathbb{R}^{n} \times \dot{\mathbb{R}}
$$

and

$$
\mathscr{R}(v, \rho) v:=m(v, \rho) \partial_{n+1} v^{ \pm}
$$

and consider

$$
\begin{cases}\partial_{t} v+\mathscr{A}(\rho) v+\mathscr{R}(v, \rho) v=0 & \text { in }(0, T) \times \mathbb{R}^{n} \times \dot{\mathbb{R}}, \\ v^{ \pm}+\operatorname{div}\left(\frac{\nabla \rho}{\sqrt{1+|\nabla \rho|^{2}}}\right)=0 & \text { on }[0, T) \times \mathbb{R}^{n}, \\ \partial_{t} \rho+[\mathscr{B}(\rho) v]=0 & \text { on }(0, T) \times \mathbb{R}^{n}, \\ v(0, \cdot)=v_{0} & \text { in } \mathbb{R}^{n} \times \mathbb{R}, \\ \rho(0, \cdot)=\rho_{0} & \text { on } \mathbb{R}^{n} .\end{cases}
$$


We call a pair $(v, \rho)$ an $L_{p}$-solution to (7.6) on $J:=[0, T]$ if

$$
\begin{aligned}
& v \in H_{p}^{1}\left(J ; L_{p}\left(\mathbb{R}^{n} \times \dot{\mathbb{R}}\right)\right) \cap L_{p}\left(J ; H_{p}^{2}\left(\mathbb{R}^{n} \times \dot{\mathbb{R}}\right)\right), \\
& \rho \in B_{p p}^{3 / 2-1 / 2 p}\left(J ; L_{p}\left(\mathbb{R}^{n}\right)\right) \cap B_{p p}^{1-1 / 2 p}\left(J ; H_{p}^{2}\left(\mathbb{R}^{n}\right)\right) \cap L_{p}\left(J ; B_{p p}^{4-1 / p}\left(\mathbb{R}^{n}\right)\right),
\end{aligned}
$$

and if $(v, \rho)$ satisfies $(7.6)$ in the $L_{p}$-sense. Any $L_{p}$-solution $(v, \rho)$ to (7.6) satisfying

$$
\rho \in C^{\infty}\left((0, T), B U C^{\infty}\left(\mathbb{R}^{n}\right)\right), \quad v \in C^{\infty}\left((0, T) \times \mathbb{R}^{n} \times \dot{\mathbb{R}}\right), \quad v^{ \pm}(t) \in B U C^{\infty}\left(\mathbb{R}_{ \pm}^{n+1}\right)
$$

for $t \in(0, T)$, is called a smooth solution. A pair $(u, \rho)$, where

$$
u: \bigcup_{t \in J}\left(\{t\} \times \Omega_{\rho(t)}\right) \rightarrow \mathbb{R}, \quad \rho: J \times \mathbb{R}^{n} \rightarrow \mathbb{R}
$$

is called an $L_{p}$-solution to (7.5) if $\left(\theta_{\rho}^{*} u, \rho\right)$ is an $L_{p}$-solution to (7.6). Finally, a pair $(u, \rho)$ is said to be a smooth solution on $[0, T]$ to (7.5) if $\rho \in C^{\infty}\left((0, T) \times \mathbb{R}^{n}\right)$, and if given $t \in(0, T)$ and $z \in \Omega_{\rho(t)}^{ \pm}$, we have $u^{ \pm}(t, \cdot) \in B U C^{\infty}\left(\Omega_{\rho(t)}^{ \pm}\right), u^{ \pm}(\cdot, z)$ is smooth in a neighborhood of $t$ and $(u, \rho)$ satisfies (7.5) pointwise.

Lemma 7.2. (a) Assume that $(v, \rho)$ is a smooth $L_{p}$-solution to (7.6). Then there exists a smooth $L_{p}$-solution to (7.5).

(b) If $L_{p}$-solutions to (7.6) are unique then so are $L_{p}$-solutions to (7.5).

Proof. (i) Let $(v, \rho)$ be a smooth $L_{p}$-solution to (7.6) and set

$$
u(t,(x, y)):=\left(\theta_{*}^{\rho}(t) v(t)\right)(x, y), \quad t \in J,(x, y) \in \Omega_{\rho(t)} .
$$

By definition, $(u, \rho)$ is an $L_{p}$-solution to (7.5). Let us verify that $(u, \rho)$ is a smooth solution to (7.5). For this fix $t_{0} \in(0, T)$ and $z_{0} \in \Omega_{\rho(t)}$. From (7.4) we conclude that $u^{ \pm}\left(t_{0}, \cdot\right) \in B U C^{\infty}\left(\Omega_{\rho\left(t_{0}\right)}^{ \pm}\right)$.

(ii) Write $z_{0}=\left(x_{0}, y_{0}\right)$ with $x_{0} \in \mathbb{R}^{n}$ and observe that

$$
\left(\theta_{\rho(t)}^{-1}\left(z_{0}\right)\right)_{i}=\left(x_{0}\right)_{i} \text { for } i=1, \ldots, n
$$

Moreover, by the smoothness of $\rho$, the implicit function theorem ensures that there is an $\varepsilon>0$ such that

$$
\psi:=\left[t \mapsto\left(\theta_{\rho(t)}^{-1}\left(z_{0}\right)\right)_{n+1}\right] \in C^{\infty}\left(I_{\varepsilon}, \mathbb{R}\right)
$$

where $I_{\varepsilon}:=\left(t_{0}-\varepsilon, t_{0}+\varepsilon\right)$, and that

$$
\frac{d}{d t}\left[\left(\theta_{\rho(t)}^{-1}\left(z_{0}\right)\right)_{n+1}\right]=-\frac{\varphi(\psi(t)) \partial_{t} \rho(t)\left(x_{0}\right)}{1+\varphi^{\prime}(\psi(t)) \rho(t)\left(x_{0}\right)}, \quad t \in I_{\varepsilon} .
$$

Hence $\left[t \mapsto u\left(t, z_{0}\right)=v(t)\left(\theta_{\rho(t)}^{-1}\left(z_{0}\right)\right)\right]$ is smooth on $I_{\varepsilon}$ with 


$$
\begin{aligned}
\partial_{t} u\left(t, z_{0}\right) & =\partial_{t} v(t)\left(\theta_{\rho(t)}^{-1}\left(z_{0}\right)\right)+\left(\nabla v(t)\left(\theta_{\rho(t)}^{-1}\left(z_{0}\right)\right) \mid \frac{d}{d t}\left(\theta_{\rho(t)}^{-1}\left(z_{0}\right)\right)\right) \\
& =\left(\theta_{*}^{\rho(t)} \partial_{t} v(t)\right)\left(z_{0}\right)-\frac{\varphi(\psi(t)) \partial_{t} \rho\left(x_{0}\right)}{1+\varphi^{\prime}(\psi(t)) \rho(t)\left(x_{0}\right)} \partial_{n+1} v(t)\left(\theta_{\rho(t)}^{-1}\left(z_{0}\right)\right) \\
& =\left(\theta_{*}^{\rho(t)}\left[\partial_{t} v(t)+m(v, \rho) \partial_{n+1} v(t)\right]\right)\left(z_{0}\right),
\end{aligned}
$$

by (7.7), (7.8) and the definition of $\psi$ and $m(v, \rho)$. Now assertion (a) follows without any difficulty. Assertion (b) is obvious.

In the following, we fix $\rho \in B_{p p}^{4-3 / p}\left(\mathbb{R}^{n}\right)$ with $\|\rho\|_{L_{\infty}}<a$. Let

$$
g_{j k}(\rho):=\left(\partial_{j} \theta_{\rho} \mid \partial_{k} \theta_{\rho}\right), \quad 1 \leqq j, k \leqq n+1
$$

denote the components of the metric tensor induced by $\theta_{\rho}$. Moreover, let $g(\rho):=\sqrt{\operatorname{det}\left[g_{j k}(\rho)\right]}$ and write $\left[g^{j k}(\rho)\right]$ for the inverse of $\left[g_{j k}(\rho)\right]$. Then one has $g(\rho)=\left(1+\varphi^{\prime} \rho\right)$ and

$$
\left[g^{j k}(\rho)\right]=\left[\begin{array}{ccccc}
1 & 0 & \cdots & 0 & \frac{-\varphi \partial_{1} \rho}{1+\varphi^{\prime} \rho} \\
0 & 1 & \cdots & 0 & \frac{-\varphi \partial_{2} \rho}{1+\varphi^{\prime} \rho} \\
\vdots & & \ddots & & \vdots \\
0 & 0 & \cdots & 1 & \frac{-\varphi \partial_{n} \rho}{1+\varphi^{\prime} \rho} \\
\frac{-\varphi \partial_{1} \rho}{1+\varphi^{\prime} \rho} & \frac{-\varphi \partial_{2} \rho}{1+\varphi^{\prime} \rho} & \cdots & \frac{-\varphi \partial_{n} \rho}{1+\varphi^{\prime} \rho} & \frac{1+|\varphi \nabla \rho|^{2}}{\left(1+\varphi^{\prime} \rho\right)^{2}}
\end{array}\right] .
$$

Using this notation we have

$$
\begin{aligned}
& \mathscr{A}(\rho) v=-\frac{1}{g(\rho)} \sum_{j, k=1}^{n+1} \partial_{j}\left(g(\rho) g^{j k}(\rho) \partial_{k} v^{ \pm}\right), \\
& {[\mathscr{B}(\rho) v]=-\left(1+|\nabla \rho|^{2}\right) \gamma\left(\partial_{n+1}\left(v^{+}-v^{-}\right)\right)+\sum_{j=1}^{n} \partial_{j} \rho \gamma\left(\partial_{j}\left(v^{+}-v^{-}\right)\right),}
\end{aligned}
$$

for $v \in H_{p}^{2}\left(\mathbb{R}^{n} \times \dot{\mathbb{R}}\right)$. To shorten our notation, let

$$
\begin{aligned}
\mathbb{E}_{1}(J):= & \left(H_{p}^{1}\left(J ; L_{p}\left(\mathbb{R}^{n} \times \dot{\mathbb{R}}\right)\right) \cap L_{p}\left(J ; H_{p}^{2}\left(\mathbb{R}^{n} \times \dot{\mathbb{R}}\right)\right)\right) \\
& \times\left(B_{p p}^{3 / 2-1 / 2 p}\left(J ; L_{p}\left(\mathbb{R}^{n}\right)\right) \cap B_{p p}^{1-1 / 2 p}\left(J ; H_{p}^{2}\left(\mathbb{R}^{n}\right)\right) \cap L_{p}\left(J ; B_{p p}^{4-1 / p}\left(\mathbb{R}^{n}\right)\right)\right), \\
\mathbb{F}_{0}(J):= & L_{p}\left(J ; L_{p}\left(\mathbb{R}^{n} \times \dot{\mathbb{R}}\right)\right) \times\left(B_{p p}^{1-1 / 2 p}\left(J ; L_{p}\left(\mathbb{R}^{n}\right)\right) \cap L_{p}\left(J ; B_{p p}^{2-1 / p}\left(\mathbb{R}^{n}\right)\right)\right) \\
& \times\left(B_{p p}^{1 / 2-1 / 2 p}\left(J ; L_{p}\left(\mathbb{R}^{n}\right)\right) \cap L_{p}\left(J ; B_{p p}^{1-1 / p}\left(\mathbb{R}^{n}\right)\right)\right) .
\end{aligned}
$$


Furthermore, let

$$
V_{a}(J):=\left\{(v, \rho) \in \mathbb{E}_{1}(J) ;\|\rho\|_{C\left(J, B U C\left(\mathbb{R}^{n}\right)\right)}<a\right\}
$$

and define

$$
\begin{aligned}
F(v, \rho) & :=(\mathscr{A}(0)-\mathscr{A}(\rho)-\mathscr{R}(v, \rho)) v \\
G(\rho) & :=\frac{1}{\sqrt{1+|\nabla \rho|^{2}}} \sum_{j, k=1}^{n}\left(\left(\sqrt{1+|\nabla \rho|^{2}}-1\right) \delta_{j k}+\frac{\partial_{j} \rho \partial_{k} \rho}{1+|\nabla \rho|^{2}}\right) \partial_{j} \partial_{k} \rho, \\
H(v, \rho) & :=[\mathscr{B}(0) v]-[\mathscr{B}(\rho) v]
\end{aligned}
$$

for $(v, \rho) \in V_{a}(J)$. Clearly, we have

$$
\operatorname{div}\left(\frac{\nabla \rho}{\sqrt{1+|\nabla \rho|^{2}}}\right)=\Delta \rho-G(\rho) .
$$

With these notations, problem (7.6) can be restated as

$$
\begin{cases}\partial_{t} v-\Delta v=F(v, \rho) & \text { in }(0, T) \times \mathbb{R}^{n} \times \dot{\mathbb{R}}, \\ \gamma v^{ \pm}+\Delta \rho=G(\rho) & \text { on }[0, T) \times \mathbb{R}^{n}, \\ \partial_{t} \rho-\left[\partial_{y} v\right]=H(v, \rho) & \text { on }(0, T) \times \mathbb{R}^{n}, \\ v(0, \cdot)=v_{0} & \text { in } \mathbb{R}^{n} \times \dot{\mathbb{R}}, \\ \rho(0, \cdot)=\rho_{0} & \text { on } \mathbb{R}^{n} .\end{cases}
$$

Finally, we set

$$
E_{1,0}:=B_{p p}^{2-2 / p}\left(\mathbb{R}^{n} \times \dot{\mathbb{R}}\right) \times B_{p p}^{4-3 / p}\left(\mathbb{R}^{n}\right),
$$

and

$$
\begin{aligned}
\mathbb{E}_{0}(J):=\{( & \left.(f, g, h),\left(w_{0}, \sigma_{0}\right)\right) \in \mathbb{F}_{0}(J) \times E_{1,0} ; \\
& \left.\gamma w_{0}^{ \pm}+\Delta \sigma_{0}=g(0),\left[\partial_{y} w_{0}\right]+h(0) \in B_{p p}^{2-6 / p}\left(\mathbb{R}^{n}\right)\right\},
\end{aligned}
$$

where $\mathbb{E}_{0}(J)$ is equipped with the natural norm

$$
\left\|\left(f, g, h, w_{0}, \sigma_{0}\right)\right\|_{\mathbb{E}_{0}(J)}:=\|(f, g, h)\|_{\mathbb{F}_{0}(J)}+\left\|\left(w_{0}, \sigma_{0}\right)\right\|_{E_{1,0}^{ \pm}}+\left\|\left[\partial_{y} w_{0}\right]+h(0)\right\|_{B_{p p}^{2-6 / p}\left(\mathbb{R}^{n}\right)} .
$$

Let

$$
\mathscr{L}: \mathbb{E}_{0}(J) \rightarrow \mathbb{E}_{1}(J), \quad\left((f, g, h),\left(w_{0}, \sigma_{0}\right)\right) \mapsto(w, \sigma)
$$

be the solution operator according to Theorem 6.1. More precisely, given

$$
\left((f, g, h),\left(w_{0}, \sigma_{0}\right)\right) \in \mathbb{E}_{0}(J)
$$


let

$$
(w, \rho)=\mathscr{L}\left(\left((f, g, h),\left(w_{0}, \sigma_{0}\right)\right)\right) \in \mathbb{E}_{1}(J)
$$

be the solution of (6.1).

Lemma 7.3. $\mathbb{E}_{0}(J)$ is a Banach space and $\mathscr{L} \in \operatorname{Isom}\left(\mathbb{E}_{0}(J), \mathbb{E}_{1}(J)\right)$.

Proof. Let $\left(\varphi_{k}\right)$ with $\varphi_{k}:=\left(f_{k}, g_{k}, h_{k}, w_{0, k}, \sigma_{0, k}\right)$ be a Cauchy sequence in $\mathbb{E}_{0}(J)$. Then there is a $\varphi=\left(f, g, h, w_{0}, \sigma_{0}\right) \in \mathbb{F}_{0}(J) \times E_{1,0}$ such that $\left(\varphi_{k}\right)$ converges in $\mathbb{F}_{0}(J) \times E_{1,0}$ toward $\varphi$. The trace theorem and Remark 5.3 (e) immediately imply that $\gamma w_{0}^{ \pm}+\Delta \sigma_{0}=g(0)$ and that $\psi_{k}:=\left[\partial_{y} w_{0, k}\right]+h_{k}(0)$ converges in $B_{p p}^{1-3 / p}\left(\mathbb{R}^{n}\right)$ to $\left[\partial_{y} w_{0}\right]+h(0)$. But by assumption $\left(\psi_{k}\right)$ is a Cauchy sequence in $B_{p p}^{2-6 / p}\left(\mathbb{R}^{n}\right)$, so that $\left[\partial_{y} w_{0}\right]+h(0) \in B_{p p}^{2-6 / p}\left(\mathbb{R}^{n}\right)$. This proves the first assertion. The second follows from Theorem 6.1.

To economize our notation, let us introduce the following convention: We write $X \cdot Y \hookrightarrow Z$ if $X, Y, Z$ are function spaces such that the pointwise multiplication induces a bounded bilinear mapping from $X \times Y$ to $Z$. Moreover, we write $C^{\omega}$ to indicate real analytic mappings.

Lemma 7.4. We have $(F, G, H) \in C^{\omega}\left(V_{a}(J), \mathbb{F}_{0}(J)\right)$ and its Fréchet derivative vanishes at 0 , that is,

$$
D(F, G, H)(0,0)=0 \quad \text { in } \mathscr{B}\left(\mathbb{E}_{1}(J), \mathbb{F}_{0}(J)\right)
$$

Proof. (i) Given $j \in\{1, \ldots, n+1\}$, we have that

$$
\begin{aligned}
\gamma \partial_{j} \in \mathscr{B}( & H_{p}^{1}\left(J ; L_{p}\left(\mathbb{R}^{n} \times \dot{\mathbb{R}}\right)\right) \cap L_{p}\left(J ; H_{p}^{2}\left(\mathbb{R}^{n} \times \dot{\mathbb{R}}\right)\right), \\
& \left.B_{p p}^{1 / 2-1 / 2 p}\left(J ; L_{p}\left(\mathbb{R}^{n}\right)\right) \cap L_{p}\left(J ; B_{p p}^{1-1 / p}\left(\mathbb{R}^{n}\right)\right)\right) .
\end{aligned}
$$

Indeed for $j=n+1$ this follows from Proposition 5.2. In case $j \in\{1, \ldots, n\}$, observe that $\gamma \partial_{j}=\partial_{j} \gamma$ and use (5.5) and Proposition 5.1. Furthermore, writing $V_{a}^{2}(J)$ for the second component of $V_{a}(J)$, we have

$$
\left[\rho \mapsto|\nabla \rho|^{2}\right] \in C^{\omega}\left(V_{a}^{2}(J), B_{p p}^{1-1 / 2 p}\left(J ; H_{p}^{1}\left(\mathbb{R}^{n}\right)\right)\right) .
$$

Using Remark 4.2 in [2] and Sobolev's embedding we conclude that

$$
B_{p p}^{1-1 / 2 p}\left(J ; H_{p}^{1}\left(\mathbb{R}^{n}\right)\right) \cdot X \hookrightarrow X
$$

holds true, where $X:=B_{p p}^{1 / 2-1 / 2 p}\left(J ; L_{p}\left(\mathbb{R}^{n}\right)\right) \cap L_{p}\left(J ; B_{p p}^{1-1 / p}\left(\mathbb{R}^{n}\right)\right)$. Thus the regularity assertion for $H$ follows from (7.12).

(ii) It follows from (5.6) that

$$
B_{p p}^{1-1 / 2 p}\left(J ; H_{p}^{2}\left(\mathbb{R}^{n}\right)\right) \cap L_{p}\left(J ; B_{p p}^{4-1 / p}\left(\mathbb{R}^{n}\right)\right) \hookrightarrow B U C\left(J ; B_{p p}^{4-3 / p}\right) .
$$

Thus Sobolev's embedding theorem implies that 


$$
\left[\rho \mapsto \sqrt{1+|\nabla \rho|^{2}}\right] \in C^{\omega}\left(V_{a}^{2}(J), B_{p p}^{1-1 / 2 p}\left(J ; B U C\left(\mathbb{R}^{n}\right)\right) \cap B U C\left(J ; B U C^{2}\left(\mathbb{R}^{n}\right)\right)\right) .
$$

Moreover, Theorem 2.8.3 in [51] and Remark 4.2 in [2] show that

$$
B_{p p}^{1-1 / 2 p}\left(J ; B U C\left(\mathbb{R}^{n}\right)\right) \cdot B_{p p}^{1-1 / 2 p}\left(J ; L_{p}\left(\mathbb{R}^{n}\right)\right) \hookrightarrow B_{p p}^{1-1 / 2 p}\left(J ; L_{p}\left(\mathbb{R}^{n}\right)\right)
$$

and

$$
B U C\left(J ; B U C^{2}\left(\mathbb{R}^{n}\right)\right) \cdot L_{p}\left(J ; B_{p p}^{2-1 / p}\left(\mathbb{R}^{n}\right)\right) \hookrightarrow L_{p}\left(J ; B_{p p}^{2-1 / p}\left(\mathbb{R}^{n}\right)\right)
$$

respectively. Combining these facts the regularity assertion for $G$ follows.

(iii) To verify the regularity of $F$, observe that

$$
\begin{gathered}
{\left[\rho \mapsto \frac{1}{\left(1+\varphi^{\prime} \rho\right)}\right] \in C^{\omega}\left(V_{a}^{2}(J), B U C\left(J ; B U C\left(\mathbb{R}_{ \pm}^{n+1}\right)\right)\right),} \\
{\left[\rho \mapsto\left(1+\varphi^{\prime} \rho\right)\right] \in C^{\omega}\left(V_{a}^{2}(J), B U C\left(J ; B U C^{1}\left(\mathbb{R}_{ \pm}^{n+1}\right)\right)\right),} \\
{\left[\rho \mapsto g^{j k}(\rho)\right] \in C^{\omega}\left(V_{a}^{2}(J), B U C\left(J ; B U C^{1}\left(\mathbb{R}_{ \pm}^{n+1}\right)\right)\right) .}
\end{gathered}
$$

Obviously, we have

$$
B U C\left(J ; B U C\left(\mathbb{R}_{ \pm}^{n+1}\right)\right) \cdot L_{p}\left(J ; L_{p}\left(\mathbb{R}_{ \pm}^{n+1}\right)\right) \hookrightarrow L_{p}\left(J ; L_{p}\left(\mathbb{R}_{ \pm}^{n+1}\right)\right)
$$

and (5.6) shows that

$$
B_{p p}^{1 / 2-1 / 2 p}\left(J ; L_{p}\left(\mathbb{R}^{n}\right)\right) \cap L_{p}\left(J ; B_{p p}^{1-1 / p}\left(\mathbb{R}^{n}\right)\right) \hookrightarrow B U C\left(J ; B U C\left(\mathbb{R}^{n}\right)\right) .
$$

Recalling the results already proved in (i), we conclude that $F$ is analytic.

(iv) Based on (7.9) the second assertion follows by direct calculations.

In the following, $\|\mathscr{L}\|$ denotes the norm of the solution operator $\mathscr{L} \in \mathscr{B}\left(\mathbb{E}_{0}(J), \mathbb{E}_{1}(J)\right)$ and $\kappa\left(\rho_{0}\right)$ denotes the sum of the principal curvatures of the hypersurface $\Gamma_{0}=\operatorname{graph}\left(\rho_{0}\right)$ for any $\rho_{0}$ in $B U C^{2}\left(\mathbb{R}^{n}\right)$; recall that $\kappa\left(\rho_{0}\right)=-\operatorname{div}\left(\frac{\nabla \rho_{0}}{\sqrt{1+\left|\nabla \rho_{0}\right|^{2}}}\right)$.

Theorem 7.5. There exists a constant $\varepsilon>0$ such that (7.11) has a unique solution $(v, \rho) \in \varepsilon \mathbb{B}_{\mathbb{E}_{1}(J)}$ for every initial value $\left(v_{0}, \rho_{0}\right) \in B_{p p}^{2-2 / p}\left(\mathbb{R}^{n} \times \dot{\mathbb{R}}\right) \times B_{p p}^{4-3 / p}\left(\mathbb{R}^{n}\right)$ satisfying the compatibility conditions

$$
\gamma v_{0}^{ \pm}=\kappa\left(\rho_{0}\right), \quad\left[\mathscr{B}\left(\rho_{0}\right) v_{0}\right] \in B_{p p}^{2-6 / p}\left(\mathbb{R}^{n}\right)
$$

and the smallness conditions

$$
\left\|\left(v_{0}, \rho_{0}\right)\right\|_{E_{1,0}} \leqq \varepsilon /(4\|\mathscr{L}\|), \quad\left\|\left[\mathscr{B}\left(\rho_{0}\right) v_{0}\right]\right\|_{B_{p p}^{2-6 / p}\left(\mathbb{R}^{n}\right)} \leqq \varepsilon /(4\|\mathscr{L}\|)
$$


Proof. For simplicity write

$$
K(v, \rho):=(F(v, \rho), G(\rho), H(v, \rho)), \quad(v, \rho) \in V_{a}(J)
$$

and observe that

$$
K \in C^{\omega}\left(V_{a}(J), \mathbb{F}_{0}(J)\right), \quad K(0,0)=0 \quad \text { and } \quad D K(0,0)=0,
$$

see Lemma 7.4. Pick $\varepsilon>0$ such that

$$
\|D K(v, \rho)\|_{\mathscr{B}\left(\mathbb{E}_{1}(J), \mathbb{F}_{0}(J)\right)} \leqq 1 /(2\|\mathscr{L}\|), \quad(v, \rho) \in \varepsilon \mathbb{B}_{\mathbb{E}_{1}(J)}
$$

We now set

$$
W(J):=\left\{(v, \rho) \in \mathbb{E}_{1}(J) ;(v(0), \rho(0))=\left(v_{0}, \rho_{0}\right),\|(v, \rho)\|_{\mathbb{E}_{1}(J)} \leqq \varepsilon\right\}
$$

and

$$
\Phi: W(J) \rightarrow \mathbb{E}_{1}(J), \quad(v, \rho) \mapsto \mathscr{L}\left(K(v, \rho),\left(v_{0}, \rho_{0}\right)\right)
$$

Note that (7.13) is equivalent to

$$
\gamma v_{0}^{ \pm}+\Delta \rho_{0}=G\left(\rho_{0}\right), \quad\left[\partial_{y} v_{0}\right]+H\left(v_{0}, \rho_{0}\right) \in B_{p p}^{2-6 / p}\left(\mathbb{R}^{n}\right) .
$$

It follows from Lemma 7.4 and (7.17) that

$$
\left(K(v, \rho),\left(v_{0}, \rho_{0}\right)\right) \in \mathbb{E}_{0}(J), \quad(v, \rho) \in W(J) .
$$

Hence, $\Phi$ is well-defined. Furthermore, $(v, \rho) \in W(J)$ is an $L_{p}$-solution to (7.5), or equivalently to (7.6), on $J=[0, T]$ if, and only if $(v, \rho)$ is a fixed point of $\Phi$.

Writing $(\tilde{v}, \tilde{\rho})=\Phi(v, \rho)$, it follows from the construction of the operator $\mathscr{L}$ that $(\tilde{v}(0), \tilde{\rho}(0))=\left(v_{0}, \rho_{0}\right)$. Moreover, we conclude from (7.14)-(7.17) that

$$
\begin{aligned}
\|\Phi(v, \rho)\|_{\mathbb{E}_{1}(J)} & \leqq\|\mathscr{L}\|\left\|\left(K(v, \rho),\left(v_{0}, \rho_{0}\right)\right)\right\|_{\mathbb{E}_{0}(J)} \\
& =\|\mathscr{L}\|\left(\|K(v, \rho)\|_{\mathbb{F}_{0}(J)}+\left\|\left(v_{0}, \rho_{0}\right)\right\|_{E_{1,0}}+\left\|\left[\mathscr{B}\left(\rho_{0}\right) v_{0}\right]\right\|_{B_{p p}^{2-6 / p}\left(\mathbb{R}^{n}\right)}\right) \leqq \varepsilon
\end{aligned}
$$

for any $(v, \rho) \in W(J)$. Hence $\Phi(W(J)) \subset W(J)$. Finally, pick $(v, \rho)(w, \sigma) \in W(J)$. Then

$$
\begin{aligned}
\|\Phi(v, \rho)-\Phi(w, \sigma)\|_{\mathbb{E}_{1}(J)} & =\|\mathscr{L}((K(v, \rho)-K(w, \sigma)),(0,0))\|_{\mathbb{E}_{1}(J)} \\
& \leqq\|\mathscr{L}\|\|K(v, \rho)-K(w, \sigma)\|_{\mathbb{F}_{0}(J)} \\
& \leqq(1 / 2)\|(v, \rho)-(w, \sigma)\|_{\mathbb{E}_{1}(J)} .
\end{aligned}
$$

The assertion now follows from the contraction principle. 


\section{Analyticity}

Throughout this section let $(v, \rho)$ be the unique $L_{p}$-solution to (7.6) on $J=[0, T]$ with the initial data $\left(v_{0}, \rho_{0}\right) \in B_{p p}^{2-2 / p}\left(\mathbb{R}^{n} \times \dot{\mathbb{R}}\right) \times B_{p p}^{4-3 / p}\left(\mathbb{R}^{n}\right)$ satisfying the compatibility condition (7.13). Our goal is to show that

$$
M=\bigcup_{t \in(0, T)}\left(\{t\} \times \Gamma_{\rho(t)}\right)
$$

is a real analytic manifold and that $(v, \rho)$ is in fact a smooth solution to (7.6). Again we will see that the property of maximal regularity of the linearized problem is of crucial importance.

Given $\mu \in \mathbb{R}^{n}$ and $g \in \mathscr{S}\left(\mathbb{R}^{n}\right)$, the Schwartz space over $\mathbb{R}^{n}$, let $\tau_{\mu} g$ be the translation of $g$ by $\mu$, i.e.

$$
\left(\tau_{\mu} g\right)(x):=g(x+\mu), \quad x \in \mathbb{R}^{n} .
$$

It is not difficult to verify that $\tau_{\mu} \in \mathscr{B}\left(\mathscr{S}\left(\mathbb{R}^{n}\right)\right)$ and by duality, $\tau_{\mu}$ extends to a mapping, still denoted by $\tau_{\mu}$, such that $\tau_{\mu} \in \mathscr{B}\left(\mathscr{S}^{\prime}\left(\mathbb{R}^{n}\right)\right)$. We first prove the following result, cf. [17].

Lemma 8.1. Assume that

$$
X \in\left\{B_{p p}^{s}\left(\mathbb{R}^{n}\right) ; s \in \mathbb{R}, p \in(1, \infty)\right\} \cup\left\{H_{p}^{k}\left(\mathbb{R}^{n}\right) ; k \in \mathbb{Z}, p \in(1, \infty)\right\}
$$

Then $\left\{\tau_{\mu} ; \mu \in \mathbb{R}^{n}\right\}$ is a strongly continuous group of contractions on $X$, satisfying

$$
\tau_{\mu} g-\tau_{\mu_{0}} g=\int_{0}^{1} \tau_{\mu_{0}+s\left(\mu-\mu_{0}\right)}\left(\mu-\mu_{0} \mid \nabla g\right) d s \quad \text { in } X
$$

for any $g \in \mathscr{S}\left(\mathbb{R}^{n}\right)$.

Proof. If $X$ belongs to $\left\{H_{p}^{k}\left(\mathbb{R}^{n}\right) ; k \in \mathbb{Z}, p \in(1, \infty)\right\}$ the assertion follows from the transformation theorem for Lebesgue's integral and the mean value theorem. If $X$ belongs to the Besov scale $\left\{B_{p p}^{s}\left(\mathbb{R}^{n}\right) ; s \in \mathbb{R}, p \in(1, \infty)\right\}$ the assertion follows from the $H_{p}^{k}$-result by interpolation.

We need some further preparation. For this pick $T^{*} \in(0, T)$ and choose $\delta>0$ such that $\lambda t \in J$ for $\lambda \in(1-\delta, 1+\delta)$ and $t \in I:=\left[0, T^{*}\right]$. Given $g: I \rightarrow \mathscr{S}^{\prime}\left(\mathbb{R}^{n}\right)$ and $(\lambda, \mu) \in \mathbb{R} \times \mathbb{R}^{n}$, let

$$
g_{\lambda, \mu}(t):=\tau_{t \mu} g(\lambda t) \text { for } t \in I
$$

Recall that $D_{n}$ stands for the realization of the negative Laplacian $\Delta$ in the spaces $B_{p p}^{s}\left(\mathbb{R}^{n}\right)$. Then we have

Lemma 8.2. Given $g_{0} \in B_{p p}^{k+1-3 / p}\left(\mathbb{R}^{n}\right), k=0,1$, let $g(t):=\exp \left(-t D_{n}\right) g_{0}$. Then there is a neighborhood $\Lambda$ of $(1,0)$ in $(1-\delta, 1+\delta) \times \mathbb{R}^{n}$ such that 


$$
\left[(\lambda, \mu) \mapsto g_{\lambda, \mu}\right] \in C^{\omega}\left(\Lambda, B_{p p}^{(k+1-1 / p) / 2}\left(I ; L_{p}\left(\mathbb{R}^{n}\right)\right)\right) \cap L_{p}\left(I ; B_{p p}^{k+1-1 / p}\left(\mathbb{R}^{n}\right)\right)
$$

Proof. (i) By well-known $L_{p}$-maximal regularity results for $D_{n}$ we know that

$$
g \in H_{p}^{1}\left(I ; B_{p p}^{k-1-1 / p}\left(\mathbb{R}^{n}\right)\right) \cap L_{p}\left(I ; B_{p p}^{k+1-1 / p}\left(\mathbb{R}^{n}\right)\right), \quad k=0,1,
$$

cf. the arguments outlined in the introduction. Hence Lemma 8.1 implies that

$$
g_{\lambda, \mu} \in L_{p}\left(I ; B_{p p}^{k+1-1 / p}\left(\mathbb{R}^{n}\right)\right)
$$

Moreover, the mean value theorem yields

$$
h^{-1}\left[g_{\lambda, \mu}(t+h)-g_{\lambda, \mu}(t)-h\left\{\lambda \tau_{t \mu} \frac{d}{d t} g(\lambda t)+\left(\mu \mid g_{\lambda, \mu}(t)\right)\right\}=I_{h}^{1}(t)+I_{h}^{2}(t)+I_{h}^{3}(t),\right.
$$

where

$$
\begin{aligned}
& I_{h}^{1}(t):=\lambda \int_{0}^{1} \tau_{(t+h) \mu}\left[\frac{d}{d t} g(\lambda t+\lambda s h)-\frac{d}{d t} g(\lambda t)\right] d s, \\
& I_{h}^{2}(t):=\lambda\left(\tau_{(t+h) \mu}-\tau_{t \mu}\right) \frac{d}{d t} g(\lambda t), \\
& I_{h}^{3}(t):=\int_{0}^{1}\left(\tau_{(t+s h) \mu}-\tau_{t \mu}\right)(\mu \mid \nabla g(\lambda t)) d s
\end{aligned}
$$

for a.a. $t \in I$ and all $|h|$ small enough. Due to Lemma 8.1 and (8.1) we now conclude that

$$
g_{\lambda, \mu} \in H_{p}^{1}\left(I ; B_{p p}^{k-1-1 / p}\left(\mathbb{R}^{n}\right)\right)
$$

satisfying

$$
\frac{d}{d t} g_{\lambda, \mu}(t)=\lambda \tau_{t \mu} \frac{d}{d t} g(\lambda t)+\left(\mu \mid g_{\lambda, \mu}(t)\right) \quad \text { a.e. on } I
$$

cf. the proof of Lemma 4.1 in [17].

Let now

$$
\begin{aligned}
& \mathbb{X}_{0}(I):=L_{p}\left(I ; B_{p p}^{k-1-1 / p}\left(\mathbb{R}^{n}\right)\right) \\
& \mathbb{X}_{1}(I):=H_{p}^{1}\left(I ; B_{p p}^{k-1-1 / p}\left(\mathbb{R}^{n}\right)\right) \cap L_{p}\left(I ; B_{p p}^{k+1-1 / p}\left(\mathbb{R}^{n}\right)\right) .
\end{aligned}
$$

Since $\Delta$ commutes with translations we deduce from (8.2) that $g_{\lambda, \mu}$ is the unique solution in $\mathbb{X}_{1}(I)$ to

$$
\frac{d}{d t} h-\lambda \Delta h=(\mu \mid \nabla h), \quad h(0)=g_{0} .
$$


Let now $G_{0}: \mathbb{X}_{1}(I) \times\left(\mathbb{R} \times \mathbb{R}^{n}\right) \rightarrow \mathbb{X}_{0}(I) \times B_{p p}^{2-3 / p}\left(\mathbb{R}^{n}\right)$ be defined by

$$
G_{0}(h,(\lambda, \mu)) \mapsto\left(\frac{d}{d t} h-\lambda \Delta h-(\mu \mid \nabla h), h(0)-g_{0}\right) .
$$

It is not difficult to verify that $G_{0}$ is real analytic, satisfying $G_{0}(g,(1,0))=0$ and, by maximal regularity,

$$
\partial_{1} G_{0}(g,(1,0))=\left(\frac{d}{d t}-\Delta, \operatorname{tr}\right) \in \operatorname{Isom}\left(\mathbb{X}_{1}(I), \mathbb{X}_{0}(I)\right) \times \operatorname{tr}\left(\mathbb{X}_{1}(I)\right) .
$$

Here, $\partial_{1} G_{0}$ denotes the derivative of $G_{0}$ with respect to $h$ and $\operatorname{tr}$ stands for the temporal trace operator. Thus the implicit function theorem shows that there is a neighborhood $\Lambda$ of $(1,0)$ in $\mathbb{R} \times \mathbb{R}^{n}$ such that

$$
\left[(\lambda, \mu) \mapsto g_{\lambda, \mu}\right] \in C^{\omega}\left(\Lambda, \mathbb{X}_{1}(I)\right)
$$

since $h \in \mathbb{X}_{1}(I)$ is a solution to $(8.3)$ if, and only if $G_{0}(h,(\lambda, \mu))=0$. By interpolation we have

$$
\mathbb{X}_{1}(I) \hookrightarrow B_{p p}^{(k+1-1 / p) / 2}\left(I ; L_{p}\left(\mathbb{R}^{n}\right)\right) \cap L_{p}\left(I ; B_{p p}^{k+1-1 / p}\left(\mathbb{R}^{n}\right)\right)
$$

cf. the proof of Remark 5.3 (a). This completes the proof.

Due to the compatibility conditions the main ingredients of the proof of Lemma 8.2 unfortunately cannot be applied directly to solutions of (7.6). To overcome this difficulty, we device a suitable decomposition of $(v, \rho)$. For this let in the following

$$
g(t):=\exp \left(-t D_{n}\right) G\left(\rho_{0}\right), \quad h(t):=\exp \left(-t D_{n}\right) H\left(v_{0}, \rho_{0}\right), \quad t \in J .
$$

By (7.17) we have

$$
\gamma v_{0}^{ \pm}+\Delta \rho_{0}=g(0) \quad \text { and } \quad\left[\partial_{y} v_{0}\right]+h(0) \in B_{p p}^{2-6 / p}\left(\mathbb{R}^{n}\right)
$$

Thus

$$
(w, \sigma):=\mathscr{L}\left(0, g, h, v_{0}, \rho_{0}\right) \in \mathbb{E}_{1}(J)
$$

is the unique solution to

$$
\begin{cases}\partial_{t} v-\Delta v=0 & \text { in }(0, T) \times \mathbb{R}^{n} \times \dot{\mathbb{R}} \\ \gamma v^{ \pm}+\Delta \rho=g & \text { on }[0, T) \times \mathbb{R}^{n} \\ \partial_{t} \rho-\left[\partial_{y} v\right]=h & \text { on }(0, T) \times \mathbb{R}^{n} \\ v(0, \cdot)=v_{0} & \text { in } \mathbb{R}^{n} \times \dot{\mathbb{R}} \\ \rho(0, \cdot)=\rho_{0} & \text { on } \mathbb{R}^{n}\end{cases}
$$

Given $\mu \in \mathbb{R}^{n}$ and $w_{0} \in L_{p}\left(\mathbb{R}^{n} \times \dot{\mathbb{R}}\right)$, let $\tau_{\mu} w_{0}$ be defined by

$$
\left(\tau_{\mu} w_{0}\right)(x, y):=w_{0}(x+\mu, y), \quad(x, y) \in \mathbb{R}^{n} \times \dot{\mathbb{R}} .
$$


Since $\Lambda \subset(1-\delta, 1+\delta) \times \mathbb{R}^{n}$, the functions

$$
w_{\lambda, \mu}(t):=\tau_{t \mu} w(\lambda t) \quad \text { and } \quad \sigma_{\lambda, \mu}(t):=\tau_{t \mu} \sigma(\lambda t), \quad t \in I
$$

are well-defined for all $(\lambda, \mu) \in \Lambda$.

Lemma 8.3. Given $(\lambda, \mu) \in \Lambda$, we have that $\left(w_{\lambda, \mu}, \sigma_{\lambda, \mu}\right) \in \mathbb{E}_{1}(I)$ and $\left(w_{\lambda, \mu}, \sigma_{\lambda, \mu}\right)$ is a solution to

$$
\begin{cases}\partial_{t} v-\lambda \Delta v=(\mu \mid \nabla v) & \text { in }\left(0, T^{*}\right) \times \mathbb{R}^{n} \times \dot{\mathbb{R}} \\ \gamma v^{ \pm}+\Delta \rho=g_{\lambda, \mu} & \text { on }\left[0, T^{*}\right) \times \mathbb{R}^{n}, \\ \partial_{t} \rho-\lambda\left[\partial_{y} v\right]=\lambda h_{\lambda, \mu}+(\mu \mid \nabla \rho) & \text { on }\left(0, T^{*}\right) \times \mathbb{R}^{n}, \\ v(0, \cdot)=v_{0} & \text { in } \mathbb{R}^{n} \times \dot{\mathbb{R}} \\ \rho(0, \cdot)=\rho_{0} & \text { on } \mathbb{R}^{n} .\end{cases}
$$

Proof. (i) Fix $(\lambda, \mu) \in \Lambda$. Using (8.5), Lemma 8.1, and Fubini's theorem it follows as in the proof of Lemma 8.2 that

$$
w_{\lambda, \mu} \in L_{p}\left(I, H_{p}^{2}\left(\mathbb{R}^{n} \times \dot{\mathbb{R}}\right)\right) \cap H_{p}^{1}\left(I, L_{p}\left(\mathbb{R}^{n} \times \dot{\mathbb{R}}\right)\right),
$$

satisfying

$$
\frac{d}{d t} w_{\lambda, \mu}(t)=\lambda \tau_{t \mu} \frac{d}{d t} w(\lambda t)+\left(\mu \mid \nabla w_{\lambda, \mu}(t)\right)
$$

in $L_{p}\left(\mathbb{R}^{n} \times \dot{\mathbb{R}}\right)$ a.e. on $I$.

(ii) To verify that $\sigma_{\lambda, \mu} \in B_{p p}^{1-1 / 2 p}\left(I, H_{p}^{2}\left(\mathbb{R}^{n}\right)\right)$ we use the intrinsic norm

$$
\left\{\|g\|_{L_{p}\left(I, H_{p}^{2}\left(\mathbb{R}^{n}\right)\right)}^{p}+\iint_{I} \frac{\|g(t)-g(s)\|_{H_{p}^{2}\left(\mathbb{R}^{n}\right)}^{p}}{|t-s|^{p+1 / 2}} d s d t\right\}^{1 / p}
$$

on $B_{p p}^{1-1 / 2 p}\left(I, H_{p}^{2}\left(\mathbb{R}^{n}\right)\right)$. Again, by Lemma 8.1 and $(8.5)$, it follows that

$$
\left\|\sigma_{\lambda, \mu}\right\|_{L_{p}\left(I ; H_{p}^{2}\left(\mathbb{R}^{n}\right)\right)} \leqq C\|\sigma\|_{L_{p}\left(J ; H_{p}^{2}\left(\mathbb{R}^{n}\right)\right)}<\infty .
$$

Moreover, we have

$$
\begin{aligned}
\left\|\sigma_{\lambda, \mu}(t)-\sigma_{\lambda, \mu}(s)\right\|_{H_{p}^{2}}^{p} & \leqq C\left(\left\|\tau_{t \mu}(\sigma(\lambda t)-\sigma(\lambda s))\right\|_{H_{p}^{2}}^{p}+\left\|\left(\tau_{t \mu}-\tau_{s \mu}\right) \sigma(\lambda s)\right\|_{H_{p}^{2}}^{p}\right) \\
& \leqq C\left(\|\sigma(\lambda t)-\sigma(\lambda s)\|_{H_{p}^{2}}^{p}+\left\|\left(\tau_{t \mu}-\tau_{s \mu}\right) \sigma(\lambda s)\right\|_{H_{p}^{2}}^{p}\right)
\end{aligned}
$$

and, recalling (8.5),

$$
\iint_{I} \frac{\|\sigma(\lambda t)-\sigma(\lambda s)\|_{H_{p}^{2}\left(\mathbb{R}^{n}\right)}^{p}}{|t-s|^{p+1 / 2}} d s d t<\infty .
$$


Finally, using Lemma 8.1, we see that

$$
\begin{aligned}
\left\|\left(\tau_{t \mu}-\tau_{s \mu}\right) \sigma(\lambda s)\right\|_{H_{p}^{2}}^{p} & =\left\|\int_{0}^{1} \tau_{(s+r(t-s)) \mu}(t-s)(\mu \mid \nabla \sigma(\lambda s)) d r\right\|_{H_{p}^{2}}^{p} \\
& \leqq|t-s|^{p}\|(\mu \mid \nabla \sigma(\lambda s))\|_{H_{p}^{2}}^{p} .
\end{aligned}
$$

By (8.5) we know that

$$
(\mu \mid \nabla \sigma(\lambda s)) \in L_{p}\left(I, B_{p p}^{3-1 / p}\left(\mathbb{R}^{n}\right)\right) \hookrightarrow L_{p}\left(I, H_{p}^{2}\left(\mathbb{R}^{n}\right)\right)
$$

Thus

$$
\int_{I} \int_{I} \frac{\left\|\left(\tau_{t \mu}-\tau_{s \mu}\right) \sigma(\lambda s)\right\|_{H_{p}^{2}\left(\mathbb{R}^{n}\right)}^{p}}{|t-s|^{p+1 / 2}} d s d t \leqq \int_{I} \int_{I} \frac{\|(\mu \mid \nabla \sigma(\lambda s))\|_{H_{p}^{2}\left(\mathbb{R}^{n}\right)}^{p}}{|t-s|^{1 / 2}} d s d t<\infty .
$$

This shows that $\sigma_{\lambda, \mu}$ belongs to the space $B_{p p}^{1-1 / 2 p}\left(I, H_{p}^{2}\left(\mathbb{R}^{n}\right)\right)$. Similarly, one verifies that $\sigma_{\lambda, \mu} \in B_{p p}^{3 / 2-1 / 2 p}\left(I, L_{p}\left(\mathbb{R}^{n}\right)\right)$, satisfying

$$
\frac{d}{d t} \sigma_{\lambda, \mu}(t)=\lambda \tau_{t \mu} \frac{d}{d t} \sigma(\lambda t)+\left(\mu \mid \nabla \sigma_{\lambda, \mu}(t)\right)
$$

(iv) Finally, observe that $\tau_{\mu_{0}}\left(\Delta w_{0}\right)=\Delta\left(\tau_{\mu_{0}} w_{0}\right)$ and $\tau_{\mu_{0}}\left[\partial_{y} w_{0}\right]=\left[\partial_{y}\left(\tau_{\mu_{0}} w_{0}\right)\right]$ for any $\mu_{0} \in \mathbb{R}^{n}$ and $w_{0} \in H_{p}^{2}\left(\mathbb{R}^{n} \times \dot{\mathbb{R}}\right)$. Combining these facts with $(8.8)$, (8.9), we conclude that $\left(w_{\lambda, \mu}, \sigma_{\lambda, \mu}\right)$ is in fact a solution to $(8.7)$.

Lemma 8.4. There exists a neighborhood $\Lambda \subset(1-\delta, 1+\delta) \times \mathbb{R}^{n}$ of $(1,0)$ such that

$$
\left[(\lambda, \mu) \mapsto\left(w_{\lambda, \mu}, \sigma_{\lambda, \mu}\right)\right] \in C^{\omega}\left(\Lambda, \mathbb{E}_{1}(I)\right)
$$

Proof. (i) Let $\Lambda_{0} \subset(1-\delta, 1+\delta) \times \mathbb{R}^{n}$ be an open neighborhood of $(1,0)$ and define $\Psi: \mathbb{E}_{1}(I) \times \Lambda_{0} \rightarrow \mathbb{E}_{0}(I)$ by

$$
\Psi((v, \rho),(\lambda, \mu)):=\left(\begin{array}{c}
\partial_{t} v-\lambda \Delta v-(\mu \mid \nabla v) \\
\gamma v^{ \pm}+\Delta \rho-g_{\lambda, \mu} \\
\frac{1}{\lambda} \partial_{t} \rho-\left[\partial_{y} v\right]-h_{\lambda, \mu}-\frac{1}{\lambda}(\mu \mid \nabla \rho) \\
v(0)-v_{0} \\
\rho(0)-\rho_{0}
\end{array}\right) .
$$

Let us first verify that $\Psi$ is well-defined. Given $((v, \rho),(\lambda, \mu)) \in \mathbb{E}_{1}(I)$, write for simplicity $\Psi_{0}:=\Psi((v, \rho),(\lambda, \mu))$. We have to show that $\Psi_{0} \in \mathbb{E}_{0}(I)$. It is clear that $\Psi_{0} \in \mathbb{F}_{0}(I) \times E_{1,0}$. Furthermore, we have

$$
\gamma\left(\Psi_{0}^{4}\right)^{ \pm}+\Delta \Psi_{0}^{5}=\gamma v^{ \pm}(0)-\gamma v_{0}^{ \pm}+\Delta \rho(0)-\Delta \rho_{0},
$$

and

$$
\Psi_{0}^{2}(0)=\gamma v^{ \pm}(0)+\Delta \rho(0)-g(0)
$$


Recalling (8.4) we find that

$$
\gamma\left(\Psi_{0}^{4}\right)^{ \pm}+\Delta \Psi_{0}^{5}=\Psi_{0}^{2}(0)
$$

Moreover, we have

$$
\left[\partial_{y} \Psi_{0}^{4}\right]+\Psi_{0}^{3}(0)=\frac{1}{\lambda} \partial_{t} \rho(0)-\left[\partial_{y} v_{0}\right]-h(0)-\left(\frac{\mu}{\lambda} \mid \nabla \rho(0)\right) .
$$

Hence it follows from Remarks 5.3 (b), (e) and (8.4) that

$$
\left[\partial_{y} \Psi_{0}^{4}\right]+\Psi_{0}^{3}(0) \in B_{p p}^{2-6 / p}\left(\mathbb{R}^{n}\right)
$$

Summarizing, we conclude that $\Psi_{0} \in \mathbb{E}_{0}(I)$.

(ii) Lemma 8.2 implies that $\Psi$ is analytic. Further, writing $\partial_{1} \Psi$ for the derivative of $\Psi$ with respect to $(v, \rho) \in \mathbb{E}_{1}(I)$ we find

$$
\left[\partial_{1} \Psi((v, \rho),(1,0))\right](w, \sigma)=\left(\partial_{t} w-\Delta w, \gamma w^{ \pm}+\Delta \sigma, \partial_{t} \sigma-\left[\partial_{y} w\right], w(0), \rho(0)\right)
$$

for $(v, \rho),(w, \sigma) \in \mathbb{E}_{1}(I)$. This means that $\partial_{1} \Psi((v, \rho),(1,0))=\mathscr{L}^{-1}$, showing that

$$
\partial_{1} \Psi((v, \rho),(1,0)) \in \operatorname{Isom}\left(\mathbb{E}_{1}(I), \mathbb{E}_{0}(I)\right),
$$

by Lemma 7.3. Since $\Psi((v, \rho),(\lambda, \mu))=0$ holds true if, and only if, $(v, \rho)$ is a solution in $\mathbb{E}_{1}(I)$ to $(8.7)$, the implicit function theorem gives the assertion.

In the following, let

$$
(z, \varphi):=(v, \rho)-(w, \sigma) \text { and } \quad X(I):=V_{a / 2}-(w, \sigma) .
$$

Recall that $(v, \rho) \in \varepsilon \mathbb{B}_{\mathbb{E}_{1}(I)}$. Let $C_{0}>0$ be the constant of the Sobolev embedding

$$
B_{p p}^{1-1 / 2 p}\left(I ; H_{p}^{2}\left(\mathbb{R}^{n}\right)\right) \hookrightarrow B U C\left(I ; B U C\left(\mathbb{R}^{n}\right)\right)
$$

and assume that $C_{0} \varepsilon \leqq a / 2$. Using Lemma 8.1 it is not difficult to verify that there is an open neighborhood $\Lambda$ of $(1,0)$ in $(1-\delta, 1+\delta) \times \mathbb{R}^{n}$ such that

$$
\left(z_{\lambda, \mu}, \varphi_{\lambda, \mu}\right) \in X(I) \quad \text { and } \quad X(I)+\left(w_{\lambda, \mu}, \sigma_{\lambda, \mu}\right) \subset V_{a}(I)
$$

for all $(\lambda, \mu) \in \Lambda$. Given $(\lambda, \mu) \in \Lambda$ and $(u, \chi) \in X(I)$ define

$$
\begin{aligned}
F_{\lambda, \mu}(u, \chi) & :=\lambda F\left(u+w_{\lambda, \mu}, \chi+\sigma_{\lambda, \mu}\right)+(\mu \mid \nabla u), \\
G_{\lambda, \mu}(u, \chi) & :=G\left(\chi+\sigma_{\lambda, \mu}\right)-g_{\lambda, \mu}, \\
H_{\lambda, \mu}(u, \chi) & :=H\left(u+w_{\lambda, \mu}, \chi+\sigma_{\lambda, \mu}\right)-h_{\lambda, \mu}+\left(\frac{\mu}{\lambda} \mid \nabla \chi\right) .
\end{aligned}
$$

It follows from Lemma 7.4, (8.10), and Lemma 8.4 that 


$$
\begin{aligned}
& {\left[((u, \chi),(\lambda, \mu)) \mapsto\left(F_{\lambda, \mu}(u, \chi), G_{\lambda, \mu}(u, \chi), H_{\lambda, \mu}(u, \chi)\right)\right]} \\
& \quad \in C^{\omega}\left(X(I) \times \Lambda, \mathbb{F}_{0}(I)\right) .
\end{aligned}
$$

As in Lemma 8.3 one shows that $\left(z_{\lambda, \mu}, \varphi_{\lambda, \mu}\right)$ is a solution to

$$
\begin{cases}\partial_{t} u-\lambda \Delta u=F_{\lambda, \mu}(u, \chi) & \text { in }\left(0, T^{*}\right) \times \mathbb{R}^{n} \times \dot{\mathbb{R}}, \\ \gamma u^{ \pm}+\Delta \chi=G_{\lambda, \mu}(\chi) & \text { on }\left[0, T^{*}\right) \times \mathbb{R}^{n}, \\ \frac{1}{\lambda} \partial_{t} \chi-\left[\partial_{y} u\right]=H_{\lambda, \mu}(u, \chi) & \text { on }\left(0, T^{*}\right) \times \mathbb{R}^{n}, \\ u(0, \cdot)=0 & \text { in } \mathbb{R}^{n} \times \dot{\mathbb{R}}, \\ \chi(0, \cdot)=0 & \text { on } \mathbb{R}^{n} .\end{cases}
$$

To get the analytic dependence of $\left(z_{\lambda, \mu}, \varphi_{\lambda, \mu}\right)$ with respect to $(\lambda, \mu)$ we have to introduce the spaces

$$
\begin{aligned}
{ }_{0} \mathbb{E}_{1}(I):= & \left({ }_{0} H_{p}^{1}\left(I ; L_{p}\left(\mathbb{R}^{n} \times \dot{\mathbb{R}}\right)\right) \cap L_{p}\left(I ; H_{p}^{2}\left(\mathbb{R}^{n} \times \dot{\mathbb{R}}\right)\right)\right) \\
& \times\left({ }_{0} B_{p p}^{3 / 2-1 / 2 p}\left(I ; L_{p}\left(\mathbb{R}^{n}\right)\right) \cap{ }_{0} B_{p p}^{1-1 / 2 p}\left(I ; H_{p}^{2}\left(\mathbb{R}^{n}\right)\right) \cap L_{p}\left(I ; B_{p p}^{4-1 / p}\left(\mathbb{R}^{n}\right)\right)\right), \\
{ }_{0} \mathbb{E}_{0}(I):= & L_{p}\left(I ; L_{p}\left(\mathbb{R}^{n} \times \dot{\mathbb{R}}\right)\right) \times\left({ }_{0} B_{p p}^{1-1 / 2 p}\left(I ; L_{p}\left(\mathbb{R}^{n}\right)\right) \cap L_{p}\left(I ; B_{p p}^{2-1 / p}\left(\mathbb{R}^{n}\right)\right)\right) \\
& \times\left({ }_{0} B_{p p}^{1 / 2-1 / 2 p}\left(I ; L_{p}\left(\mathbb{R}^{n}\right)\right) \cap L_{p}\left(I ; B_{p p}^{1-1 / p}\left(\mathbb{R}^{n}\right)\right)\right)
\end{aligned}
$$

where we employed the notation of (3.1). We also set ${ }_{0} X(I):=X(I) \cap_{0} \mathbb{E}_{1}(I)$. Then $\mathbb{E}_{1}(I)$ and ${ }_{0} \mathbb{E}_{0}(I)$ are closed subspaces of $\mathbb{E}_{1}$ and $\mathbb{F}_{0}(I)$, respectively, and ${ }_{0} X(I)$ is open in $\mathbb{E}_{1}(I)$.

Define now ${ }_{0} \Psi:{ }_{0} X(I) \times \Lambda \rightarrow{ }_{0} \mathbb{E}_{0}(I)$ by

$$
{ }_{0} \Psi((u, \chi),(\lambda, \mu)):=\left(\begin{array}{c}
\partial_{t} u-\lambda \Delta u-F_{\lambda, \mu}(u, \chi) \\
\gamma u^{ \pm}+\Delta \chi-G_{\lambda, \mu}(\chi) \\
\frac{1}{\lambda} \partial_{t} \chi-\left[\partial_{y} u\right]-H_{\lambda, \mu}(u, \chi)
\end{array}\right)
$$

It follows from the first relation of (8.4) and (8.11) that

$$
{ }_{0} \Psi \in C^{\omega}\left({ }_{0} X(I) \times \Lambda,{ }_{0} \mathbb{E}_{0}(I)\right)
$$

with

$$
\partial_{1}\left[{ }_{0} \Psi((u, \chi),(1,0))\right](\tilde{u}, \tilde{\chi})=\left(\begin{array}{c}
\partial_{t} \tilde{u}-\Delta \tilde{u}-D F_{1,0}(u, \chi)(\tilde{u}, \tilde{\chi}) \\
\gamma \tilde{u}^{ \pm}+\Delta \tilde{\chi}-D G_{1,0}(\chi) \tilde{\chi} \\
\partial_{t} \tilde{\chi}-\left[\partial_{y} \tilde{u}\right]-D H_{1,0}(u, \chi)(\tilde{u}, \tilde{\chi})
\end{array}\right)
$$

for $(u, \chi) \in{ }_{0} X(I)$ and $(\tilde{u}, \tilde{\chi}) \in{ }_{0} \mathbb{E}_{1}(I)$.

Let ${ }_{0} \mathscr{L}:{ }_{0} \mathbb{E}_{0}(I) \rightarrow{ }_{0} \mathbb{E}_{1}(I)$ be the solution operator according to 


$$
\begin{cases}\partial_{t} u-\Delta u=f & \text { in }\left(0, T^{*}\right) \times \mathbb{R}^{n} \times \dot{\mathbb{R}} \\ \gamma u^{ \pm}+\Delta \chi=g & \text { on }\left[0, T^{*}\right) \times \mathbb{R}^{n} \\ \partial_{t} \chi-\left[\partial_{y} u\right]=h & \text { on }\left(0, T^{*}\right) \times \mathbb{R}^{n}\end{cases}
$$

Theorem 6.1 implies that

$$
{ }_{0} \mathscr{L} \in \operatorname{Isom}\left({ }_{0} \mathbb{E}_{0}(I),{ }_{0} \mathbb{E}_{1}(I)\right) .
$$

Furthermore it follows from (8.5) that

$$
\|(w, \sigma)\|_{\mathbb{E}_{1}(I)} \rightarrow 0 \quad \text { as }\left\|\left(v_{0}, \rho_{0}\right)\right\|_{E_{1,0}} \rightarrow 0 .
$$

In particular, we may assume that $(0,0) \in X(I)$ and that, given $\varepsilon_{0}>0$, we have

$$
\left\|\left(D F_{1,0}(z, \varphi), D G_{1,0}(z, \varphi), D H_{1,0}(z, \varphi)\right)\right\|_{\left.\mathscr{B}_{\left(0 \mathbb{E}_{1}(I), 0\right.} \mathbb{E}_{0}(I)\right)}<\varepsilon_{0}
$$

Combining (8.15) and (8.16) we conclude that

$$
\partial_{1}\left[{ }_{0} \Psi((z, \varphi),(1,0))\right] \in \operatorname{Isom}\left({ }_{0} \mathbb{E}_{1}(I),{ }_{0} \mathbb{E}_{0}(I)\right) .
$$

Again it is easily verified that $(u, \chi) \in{ }_{0} X(I)$ is a solution to the system (8.12) if, and only if the equation ${ }_{0} \Psi((u, \chi),(\lambda, \mu))=0$ holds true. Thus the implicit function theorem implies that

$$
\left[(\lambda, \mu) \mapsto\left(z_{\lambda, \mu}, \varphi_{\lambda, \mu}\right)\right] \in C^{\omega}\left(\Lambda, \mathbb{E}_{1}(I)\right)
$$

Combing this result with Lemma 8.4, we obtain such that

Theorem 8.5. There exists an open neighborhood $\Lambda$ of $(1,0)$ in $(1-\delta, 1+\delta) \times \mathbb{R}^{n}$

$$
\left[(\lambda, \mu) \mapsto\left(v_{\lambda, \mu}, \rho_{\lambda, \mu}\right)\right] \in C^{\omega}\left(\Lambda, \mathbb{E}_{1}(I)\right)
$$

As an immediate consequence we get

Corollary 8.6. The function $\rho$ belongs to $C^{\omega}\left((0, T) \times \mathbb{R}^{n}\right)$.

Proof. Pick $\left(t_{0}, x_{0}\right) \in(0, T) \times \mathbb{R}^{n}$. From Remark 5.3 (a) and Theorem 8.5 we conclude that $\left[(\lambda, \mu) \mapsto \rho_{\lambda, \mu}\right] \in C^{\omega}\left(\Lambda, C\left(I, B U C\left(\mathbb{R}^{n}\right)\right)\right)$. Thus

$$
\left[(\lambda, \mu) \mapsto \rho\left(\lambda t_{0}, x_{0}+t_{0} \mu\right)\right] \in C^{\omega}(\Lambda, \mathbb{R})
$$

and the proof in now complete.

Proof of Theorem 1.1. Theorem 7.5 guarantees the existence of a unique $L_{p}$-solution $(v, \rho)$ to $(7.11)$.

Further, Corollary 8.6 implies that $M=\bigcup_{t \in(0, T)}(\{t\} \times \Gamma(t))$ is a real analytic mani- 
fold. To verify that $v^{ \pm} \in C^{\infty}\left((0, T), B U C^{\infty}\left(\mathbb{R}_{ \pm}^{n+1}\right)\right)$, consider the semilinear parabolic equation

$$
\begin{aligned}
\partial_{t} v-\mathscr{A}(\rho) v+\mathscr{R}(v, \rho) v=0 & \text { in }(0, T) \times \mathbb{R}^{n} \times \dot{\mathbb{R}} \\
\gamma v^{ \pm}+\operatorname{div}\left(\frac{\nabla \rho}{1+|\nabla \rho|^{2}}\right)=0 & \text { on }(0, T) \times \mathbb{R}^{n} \\
v(0, \cdot)=v_{0} & \text { on }(0, T) \times \mathbb{R}^{n}
\end{aligned}
$$

for the function $v$. It follows from Corollary 8.6 and (7.10) that all coefficients of $\mathscr{A}(\rho)$ and $\mathscr{R}(\cdot, \rho)$ are smooth. Of course also $\operatorname{div}\left(\nabla \rho /\left(1+|\nabla \rho|^{2}\right)\right)$ is smooth. Hence we conclude from well-known regularity for semilinear parabolic equations that $v^{ \pm}$belongs to $C^{\infty}\left((0, T), B U C^{\infty}\left(\mathbb{R}_{ \pm}^{n+1}\right)\right)$. Now Lemma 7.2 (a) completes the proof.

Remarks 8.7. (a) The above proof shows that $v^{ \pm} \in C^{\omega}\left((0, T) \times \mathbb{R}^{n} \times \dot{\mathbb{R}}\right)$ if one uses the transformation defined in (7.2). In order to be prepared to treat the case of hypersurfaces which are not graphs we preferred to work with the transformation $\theta_{\rho}$.

(b) The idea to use the implicit function theorem and maximal regularity results to get analytic dependence of solutions to abstract evolution equations goes back to Angenent [3].

\section{Appendix}

In this section we collect some important estimates that are used in the previous sections. We begin by considering the holomorphic function

$$
g(\lambda, z)=\frac{\lambda}{\lambda+z \sqrt{\lambda+z}}, \quad \lambda \in \Sigma_{\theta}, z \in \Sigma_{\eta},
$$

where $\theta+2 \eta<\pi, \quad \theta>\pi / 2$. If $\lambda \in \Sigma_{\theta}, \quad z \in \Sigma_{\eta}$ then $\lambda+z \in \Sigma_{\theta}$ as well, hence $|\arg \sqrt{\lambda+z}| \leqq \theta / 2$. If $\arg \lambda \leqq 0$ then $\arg \sqrt{\lambda+z} \geqq-\eta / 2$, and in case $\arg \lambda \geqq 0$ then $\arg \sqrt{\lambda+z} \leqq \eta / 2$. This yields

$$
\arg (z \sqrt{\lambda+z})=\arg \sqrt{\lambda+z}+\arg z \geqq-\frac{3}{2} \eta \quad \text { if } \arg \lambda \geqq 0
$$

hence there are constants $c_{1}, c_{2}>0$ such that

$$
|\lambda+z \sqrt{\lambda+z}| \geqq c_{1}[|\lambda|+|\sqrt{\lambda+z}||z|]
$$

and

$$
|\lambda+z \sqrt{\lambda+z}| \geqq c_{2}\left[|\lambda|+|\lambda|^{1 / 2}|z|+|z|^{3 / 2}\right]
$$

on $\Sigma_{\theta} \times \Sigma_{\eta}$, where $c_{1}, c_{2}$ depend only on $\theta, \eta$. 
This shows that $g(\lambda, z)$ is uniformly bounded on $\Sigma_{\theta} \times \Sigma_{\eta}$. Next we write

$$
g(\lambda, z)=\left(\frac{1}{2 \pi i}\right)^{2} \int_{\Gamma_{\lambda}} \int_{\Gamma_{z}} \frac{g(u, v)}{(u-\lambda)(v-z)} d v d u
$$

where $\Gamma_{\lambda}, \Gamma_{z}$ denote the boundary curves of $\Sigma_{\theta}, \Sigma_{\eta}$, respectively, and where $\lambda \in \Sigma_{\theta}$ and $z \in \Sigma_{\eta}$. Taking derivatives in $(\lambda, z)$ we get

$$
\frac{1}{k !} \frac{1}{l !} D_{\lambda}^{k} D_{z}^{l} g(\lambda, z)=\frac{1}{(2 \pi i)^{2}} \iint_{\Gamma_{\lambda}} \frac{g(u, v) d v d u}{(u-\lambda)^{k+1}(v-z)^{l+1}},
$$

which implies on slightly smaller sectors $\Sigma_{\theta^{\prime}}, \Sigma_{\eta^{\prime}}$ the Cauchy estimate

$$
\left|z^{l} \lambda^{k} D_{\lambda}^{k} D_{z}^{l} g(\lambda, z)\right| \leqq M_{k, l}, \quad k, l \in \mathbb{N}_{0}, \lambda \in \Sigma_{\theta^{\prime}}, z \in \Sigma_{\eta^{\prime}} .
$$

Now we are ready to prove multiplier estimates for the symbol

$$
m(\lambda, \xi)=\frac{\lambda}{\lambda+|\xi|^{2} \sqrt{\lambda+|\xi|^{2}}}
$$

In fact, with $h(\xi)=|\xi|^{2}$ we obtain

$$
m(\lambda, \xi)=g(\lambda, h(\xi))
$$

Note that $\partial_{j} h(\xi)=2 \xi_{j}, \partial_{j} \partial_{k} h(\xi)=2 \delta_{j k}, \partial_{j} \partial_{k} \partial_{l} h(\xi)=0$; hence

$$
|\xi|^{|\alpha|}\left|D^{\alpha} h(\xi)\right| \leqq 2|\xi|^{2}=2 h(\xi) \quad \text { for all } \xi \in \mathbb{R}^{n}, \alpha \in \mathbb{N}_{0}^{n}
$$

This implies with some constants $c_{\beta_{1}, \ldots, \beta_{l}}$ :

$$
D_{\lambda}^{k} D_{\xi}^{\alpha} m(\lambda, \xi)=\sum_{l=1}^{|\alpha|} \sum_{\beta_{1}, \ldots, \beta_{l}}\left[D_{\lambda}^{k} D_{z}^{l} g(\lambda, h(\xi))\right] D_{\xi}^{\beta_{1}} h(\xi) \cdots D_{\xi}^{\beta_{l}} h(\xi) \cdot c_{\beta_{1}, \ldots, \beta_{l}}
$$

where the sum is taken over all multi-indices $\beta_{j}$ such that $\beta_{1}+\cdots+\beta_{l}=\alpha$. Therefore

$$
\begin{aligned}
|\xi|^{|\alpha|}\left|\lambda^{k}\right|\left|D_{\lambda}^{k} D_{\xi}^{\alpha} m(\lambda, \xi)\right| & \leqq c \sum_{l=1}^{|\alpha|} \sum_{\beta_{1}, \ldots, \beta_{l}}\left|\lambda^{k} D_{\lambda}^{k} D_{z}^{l} g(\lambda, h(\xi))\right| \prod_{j=1}^{l}|\xi|^{\left|\beta_{j}\right|}\left|D_{\xi}^{\beta_{j}} h(\xi)\right| \\
& \leqq c \sum_{l=1}^{|\alpha|} \sum_{\beta_{1}, \ldots, \beta_{l}}\left|\lambda^{k} h(\xi)^{l} D_{\lambda}^{k} D_{z}^{l} g(\lambda, h(\xi))\right||\xi|^{-2 l} \prod_{j=1}^{l}\left(2|\xi|^{2}\right) \leqq M_{\alpha, k} .
\end{aligned}
$$

These estimates show that the Mikhlin-condition for $m(\lambda, \xi)$ is satisfied, separately in $\lambda$ and $\xi$. However, note that they do not hold jointly in $\lambda$ and $\xi$.

Lemma 9.1. Let $\alpha \in \mathbb{N}_{0}^{n}, k \in \mathbb{N}_{0}$, and define

$$
m(\lambda, \xi)=\frac{\lambda}{\lambda+|\xi|^{2} \sqrt{\lambda+|\xi|^{2}}}, \quad \xi \in \mathbb{R}^{n},|\arg \lambda|<\theta<\pi, \xi \neq 0, \lambda \neq 0
$$


Then there is a constant $M(\alpha, k, \theta)>0$ such that

$$
|\lambda|^{k}|\xi|^{|\alpha|}\left|D_{\lambda}^{k} D_{\xi}^{\alpha} m(\lambda, \xi)\right| \leqq M(\alpha, k, \theta), \quad \xi \in \mathbb{R}^{n}, \xi \neq 0,|\arg \lambda|<\theta .
$$

By the same type of arguments we also obtain the Mikhlin-condition for the symbols

$$
\frac{|\xi|}{\sqrt{\lambda+|\xi|^{2}}}=\sqrt{\frac{z}{\lambda+z}} \circ h(\xi) \text { and } \frac{\sqrt{\lambda}}{\sqrt{\lambda+|\xi|^{2}}}=\sqrt{\frac{\lambda}{\lambda+z}} \circ h(\xi)=1-\frac{|\xi|}{\sqrt{\lambda+|\xi|^{2}}} .
$$

Lemma 9.2. Let $\alpha \in \mathbb{N}_{0}^{n}, k \in \mathbb{N}_{0}$, and define

$$
p(\lambda, \xi)=\frac{\sqrt{\lambda}}{\sqrt{\lambda+|\xi|^{2}}}, \quad \xi \in \mathbb{R}^{n},|\arg \lambda|<\theta<\pi, \xi \neq 0, \lambda \neq 0
$$

Then there is a constant $M(\alpha, k, \theta)>0$ such that

$$
|\lambda|^{k}|\xi|^{|\alpha|}\left|D_{\lambda}^{k} D_{\xi}^{\alpha} p(\lambda, \xi)\right| \leqq M(\alpha, k, \theta), \quad \xi \in \mathbb{R}^{n},|\arg \lambda|<\theta, \xi \neq 0 \neq \lambda .
$$

The next lemma refers to the multiplier $e^{-\omega y}$ which appears in the treatment of the Dirichlet extension operator. We recall that $\omega:=\sqrt{\lambda+|\xi|^{2}}$.

Lemma 9.3. Let $\alpha \in \mathbb{N}_{0}^{n}, k \in \mathbb{N}_{0}$, and set

$$
q(\lambda, \xi, y)=e^{-\omega y}, \quad \xi \in \mathbb{R}^{n}, \lambda \in \Sigma_{\theta}, y \geqq 0 .
$$

Then there are constants $M(\alpha, k, \theta)>0$ and $c(\alpha, k, \theta)>0$ such that

$$
\begin{array}{r}
|\lambda|^{k}|\xi|^{|\alpha|}\left|D_{\lambda}^{k} D_{\xi}^{\alpha} q(\lambda, \xi, y)\right| \leqq M(\alpha, k, \theta) e^{-c(\alpha, k, \theta) \sqrt{|\lambda|}}, \\
\text { for all } \xi \in \mathbb{R}^{n},|\arg \lambda|<\theta, \xi \neq 0, \lambda \neq 0, y>0 .
\end{array}
$$

Proof. Set $h(z, y)=e^{-z y}$. Then $D_{z}^{l} h(z, y)=(-y)^{l} e^{-z y}$ for all $l \in \mathbb{N}_{0}, z \in \mathbb{C}, y>0$. This implies

$$
|z|^{l}\left|D_{z}^{l} h(z, y)\right| \leqq|z y|^{l} e^{-R e z y} \leqq c_{l, \varphi} e^{-c_{\varphi}|z| y}
$$

for all $z \in \Sigma_{\varphi}, l \in \mathbb{N}_{0}$, where $\varphi<\pi / 2$. Now, we have $q(\lambda, \xi, y)=e^{-z y} \circ \omega(\lambda, \xi)$, hence the assertion follows by induction.

We shall also need the following lemma, which is known as Hilbert's inequality.

Lemma 9.4. Let $1<p<\infty$, and define $H_{0}$ in $L_{p}\left(\mathbb{R}_{+}\right)$by

$$
\left(H_{0} \varphi\right)(t)=\int_{0}^{\infty} \varphi(s) \frac{d s}{t+s}, \quad t>0
$$

Then $H_{0} \in \mathscr{B}\left(L_{p}\left(\mathbb{R}_{+}\right)\right)$. 
Proof. Let $H$ denote the Hilbert transform on $L_{p}(\mathbb{R})$. Then $H_{0}=P_{+} R H E_{0}$, where $P_{+}$denotes the restriction to $\mathbb{R}_{+}, R$ the reflection at 0 , and $E_{0}$ the extension by zero. Since $P_{+}, R, E_{0}$ are bounded linear operators, we obtain the boundedness of $H_{0}$ from that of the Hilbert transform in $L_{p}(\mathbb{R})$.

For the sake of completeness we include the following result concerning complex interpolation, see also [24], Theorem 3.1. We refer to Prüss [42] for the terminology.

Lemma 9.5. Let $X$ be a Banach space of class $\mathscr{H} \mathscr{T}$ and assume that $A, B \in B I P(X)$ are commuting in the resolvent sense and that their power angles satisfy the parabolicity condition $\theta_{A}+\theta_{B}<\pi$. Assume further that $A$ is boundedly invertible. Then

(a) $A^{\alpha}(A+B)^{-\alpha}$ and $B^{\alpha}(A+B)^{-\alpha}$ are bounded linear operators in $X$, for each $\alpha \in(0,1)$

(b) $\mathscr{D}\left((A+B)^{\alpha}\right)=[X, \mathscr{D}(A+B)]_{\alpha}=[X, \mathscr{D}(A)]_{\alpha} \cap[X, \mathscr{D}(B)]_{\alpha}=\mathscr{D}\left(A^{\alpha}\right) \cap \mathscr{D}\left(B^{\alpha}\right)$, for all $\alpha \in(0,1)$.

Here $[X, Y]_{\alpha}$ denote the complex interpolation spaces between $X$ and $Y$.

Proof. By the theorem of Dore and Venni and the results of Prüss and Sohr [44], $A+B$ is invertible, sectorial and admits bounded imaginary powers with power angle $\theta_{A+B} \leqq \max \left\{\theta_{A}, \theta_{B}\right\}$. We therefore have $[X, \mathscr{D}(T)]_{\alpha}=\mathscr{D}\left(T^{\alpha}\right)$, for $T=A, B, A+B$, see [50], Theorem 1.15.3, or [54]. Thus

$$
\mathscr{D}\left((A+B)^{\alpha}\right)=[X, \mathscr{D}((A+B))]_{\alpha} \hookrightarrow[X, \mathscr{D}(A)]_{\alpha} \cap[X, \mathscr{D}(B)]_{\alpha}=\mathscr{D}\left(A^{\alpha}\right) \cap \mathscr{D}\left(B^{\alpha}\right) .
$$

This proves (a) as well as one inclusion of (b).

Conversely, for $x \in \mathscr{D}\left(A^{\alpha}\right) \cap \mathscr{D}\left(B^{\alpha}\right)$ we have

$$
(A+B)^{\alpha} x=(A+B)(A+B)^{\alpha-1} x=A^{1-\alpha}(A+B)^{\alpha-1} A^{\alpha} x+B^{1-\alpha}(A+B)^{\alpha-1} B^{\alpha} x,
$$

and so by (a)

$$
\left\|(A+B)^{\alpha} x\right\|_{X} \leqq C\left[\left\|A^{\alpha} x\right\|_{X}+\left\|B^{\alpha} x\right\|_{X}\right]
$$

which proves the second inclusion of (b).

The following lemma is due to Grisvard [26], even in a more general context.

Lemma 9.6. Suppose $A, B$ are sectorial linear operators in a Banach space $X$, commuting in the resolvent sense. Then

$$
(X, \mathscr{D}(A) \cap \mathscr{D}(B))_{\alpha, p}=(X, \mathscr{D}(A))_{\alpha, p} \cap(X, \mathscr{D}(B))_{\alpha, p}, \quad \alpha \in(0,1), p \in[1, \infty] .
$$

Here $(X, Y)_{\alpha, p}$ denote the real interpolation spaces between $X$ and $Y$.

The next result goes back to Sobolevskii [49]. 
Lemma 9.7 (The mixed derivative theorem). Suppose $A$ and $B$ are commuting sectorial operators in a Banach space $X$ which are coercively positive, i.e. $A+t B$ with natural domain $\mathscr{D}(A+t B)=\mathscr{D}(A) \cap \mathscr{D}(B)$ is closed for each $t>0$ and there is a constant $M>0$ such that

$$
\|A x\|_{X}+t\|B x\|_{X} \leqq M\|A x+t B x\|_{X}, \quad \text { for all } x \in \mathscr{D}(A) \cap \mathscr{D}(B), t>0 .
$$

Then there is a constant $C>0$ such that

$$
\left\|A^{\alpha} B^{1-\alpha} x\right\|_{X} \leqq C\|A x+B x\|_{X}, \quad \text { for all } x \in \mathscr{D}(A) \cap \mathscr{D}(B), \alpha \in[0,1] .
$$

In particular, if $A+B$ is invertible then $A^{\alpha} B^{1-\alpha}(A+B)^{-1}$ is bounded in $X$, for each $\alpha \in[0,1]$.

Observe that Lemma 9.7 applies in particular to the situation of the Dore-Venni theorem in the version given in Prüss and Sohr [44].

Acknowledgment. The research leading to this paper was initiated and partly carried out while the third author was visiting the Fachbereich Mathematik und Informatik at Martin-Luther-Universität Halle-Wittenberg in January-February 1999. He would like to express his gratitude to the Fachbereich for the kind hospitality and to MartinLuther-Universität for partial financial support which made this visit possible.

\section{References}

[1] R. A. Adams, Sobolev Spaces, Academic Press, New York 1975.

[2] H. Amann, Multiplication in Sobolev and Besov Spaces, in: Nonlinear Analysis, A Tribute in Honour of G. Prodi, Quaderni, Scuola Norm. Sup. Pisa (1991), 27-50.

[3] S. Angenent, Parabolic equations for curves on surfaces, Part I. Curves with $p$-integrable curvature, Ann. Math. 132 (1990), 451-483.

[4] I. Athanasopoulos, L. Caffarelli, S. Salsa, Regularity of the free boundary in parabolic phase-transition problems, Acta Math. 176 (1996), 245-282.

[5] I. Athanasopoulos, L. Caffarelli, S. Salsa, Phase transition problems of parabolic type: flat free boundaries are smooth, Comm. Pure Appl. Math. 51 (1998), 77-112.

[6] B. V. Bazalii, Stefan problem for the Laplace equation with regard for the curvature of the free boundary, Ukrain. Math. J. 49 (1997), 1465-1484.

[7] L. A. Caffarelli, The regularity of free boundaries in higher dimensions, Acta Math. 139 (1977), $155-184$.

[8] L. A. Caffarelli, Some aspects of the one-phase Stefan problem, Indiana Univ. Math. J. 27 (1978), $73-77$.

[9] L. A. Caffarelli, L. C. Evans, Continuity of the temperature in the two-phase Stefan problem, Arch. Rat. Mech. Anal. 81 (1983), 199-220.

[10] L. A. Caffarelli, A. Friedman, Continuity of the temperature in the Stefan problem, Indiana Univ. Math. J. 28 (1979), 53-70.

[11] G. Caginalp, An analysis of a phase field model of a free boundary, Arch. Rat. Mech. Anal. 92 (1986), 205-245.

[12] B. Chalmers, Principles of Solidification, Krieger, Huntington-New York 1977.

[13] X. Chen, J. Hong, F. Yi, Existence, uniqueness, and regularity of classical solutions of the Mullins-Sekerka problem, Comm. Part. Diff. Equ. 21 (1996), 1705-1727.

[14] E. DiBenedetto, Regularity properties of the solution of an $n$-dimensional two-phase Stefan problem, Boll. Un. Mat. Ital. Suppl. (1980), 129-152.

[15] E. DiBenedetto, Continuity of weak solutions to certain singular parabolic equations, Ann. Mat. Pura Appl. (4) 130 (1982), 131-176.

[16] J. Escher, G. Simonett, On Hele-Shaw models with surface tension, Math. Res. Lett. 3 (1996), 467-474.

[17] J. Escher, G. Simonett, Analyticity of the interface in a free boundary problem, Math. Ann. 305 (1996), 439-459. 
[18] J. Escher, G. Simonett, Classical solutions to Hele-Shaw models with surface tension, Adv. Diff. Equ. 2 (1997), 619-642.

[19] J. Escher, G. Simonett, A center manifold analysis for the Mullins-Sekerka model, J. Diff. Equ. 143 (1998), 267-292.

[20] A. Friedman, The Stefan problem in several space variables, Trans. Amer. Math. Soc. 133 (1968), 51-87.

[21] A. Friedman, Variational Principles and Free-Boundary Problems, Wiley-Interscience, New York 1982.

[22] A. Friedman, D. Kinderlehrer, A one phase Stefan problem, Indiana Univ. Math. J. 24 (1975), $1005-1035$.

[23] A. Friedman, F. Reitich, The Stefan problem with small surface tension, Trans. Amer. Math. Soc. 328 (1991), 465-515.

[24] M. Giga, Y. Giga, H. Sohr, $L^{p}$ estimates for the Stokes system, Functional analysis and related topics (H. Komatsu, ed.), Springer Lect. Notes Math. 1540 (1993), 55-67.

[25] D. Gilbarg, N. S. Trudinger, Elliptic Partial Differential Equations of Second Order, Springer, Berlin 1977.

[26] P. Grisvard, Spays di trace e applicazioni, Rend. Math. 5 (1972), 657-729.

[27] M. E. Gurtin, On the two phase problem with interfacial energy and entropy, Arch. Rat. Mech. Anal. 96 (1986), 199-241.

[28] M. E. Gurtin, Multiphase thermomechanics with interfacial structure, Arch. Rat. Mech. Anal. 104 (1988), 195-221.

[29] E. I. Hanzawa, Classical solutions of the Stefan problem, Tôhoku Math. J. 33 (1981), 297-335.

[30] P. Hartman, Crystal Growth: An Introduction, North-Holland, Amsterdam 1973.

[31] S. L. Kamenomostskaja, On Stefan's problem, Math. Sbornik 53 (1965), 485-514.

[32] D. Kinderlehrer, L. Nirenberg, Regularity in free boundary problems, Ann. Scuola Norm. Sup. Pisa (4) 4 (1977), 373-391.

[33] D. Kinderlehrer, L. Nirenberg, The smoothness of the free boundary in the one phase Stefan problem, Comm. Pure Appl. Math. 31 (1978), 257-282.

[34] O. A. Ladyženskaya, V. A. Solonnikov, and N. N. Ural'ceva, Linear and Quasilinear Equations of Parabolic Type, Transl. Math. Monogr. 23, Amer. Math. Soc. (1968).

[35] J. S. Langer, Instabilities and pattern formation in crystal growth, Rev. Mod. Phys. 52 (1980), 1-28.

[36] S. Luckhaus, Solutions for the two-phase Stefan problem with the Gibbs-Thomson law for melting temperature, Europ. J. Appl. Math. 1 (1990), 101-111.

[37] H. Matano, Asymptotic behavior of the free boundaries arising in one phase Stefan problems in multidimensional spaces, Lect. Notes Num. Appl. Anal. 5, Kinokuniya, Tokyo (1982), 133-151.

[38] A. M. Meirmanov, On the classical solution of the multidimensional Stefan problem for quasilinear parabolic equations, Math. Sb. 112 (1980), 170-192.

[39] A. M. Meirmanov, The Stefan Problem, De Gruyter, Berlin 1992.

[40] A. M. Meirmanov, The Stefan problem with surface tension in the three dimensional case with spherical symmetry: non-existence of the classical solution, Europ. J. Appl. Math. 5 (1994), 1-20.

[41] R. H. Nochetto, A class of nondegenerate two-phase Stefan problems in several space variables, Comm. Part. Diff. Equ. 12 (1987), 21-45.

[42] J. Prüss, Evolutionary Integral Equations and Applications, Monogr. Math. 87, Birkhäuser Verlag, Basel 1993.

[43] J. Prüss, Laplace transforms and regularity of solutions of evolutionary integral equations, preprint.

[44] J. Prüss and H. Sohr, On operators with bounded imaginary powers in Banach spaces, Math. Z. 203 (1990), 429-452.

[45] E. V. Radkevich, Gibbs-Thomson law and existence of the classical solution of the modified Stefan problem, Soviet Dokl. Acad. Sci. 316 (1991), 1311-1315.

[46] E. V. Radkevich, The Gibbs-Thompson correction and conditions for the existence of a classical solution of the modified Stefan problem (Russian), Dokl. Akad. Nauk SSSR 316 (1991), 1311-1315, Translation in Soviet Math. Dokl. 43 (1991), 274-278.

[47] L. I. Rubinstein, The Stefan Problem, Transl. Math. Monogr. 27, American Mathematical Society, Providence, R.I., 1971.

[48] P. E. Sacks, Continuity of solutions of a singular parabolic equation, Nonlin. Anal. 7 (1983), 387-409.

[49] P. E. Sobolevskii, Fractional powers of coercively positive sums of operators, Soviet Math. Dokl. 16 (1975), $1638-1641$.

[50] H. Triebel, Interpolation Theory, Function Spaces, Differential Operators, North-Holland, Amsterdam 1978.

[51] H. Triebel, Theory of Function Spaces, Monogr. Math. 78, Birkhäuser, Basel 1983.

[52] A. Visintin, Remarks on the Stefan problem with surface tension, in: Boundary value problems for partial differential equations and applications (J. L. Lions and C. Baiocchi, eds.), RMA: Res. Notes Appl. Math. 29, Masson, Paris 1993. 
[53] A. Visintin, Models of Phase Transitions, Progr. Nonlin. Diff. Equ. Appl. 28, Birkhäuser, Boston 1996.

[54] A. Yagi, Coincidence entre des espaces d'interpolation et des domaines de puissances fractionnaires d'operateurs, C.R. Acad. Sci. Paris 299 (1984), 173-176.

[55] W. P. Ziemer, Interior and boundary continuity of weak solutions of degenerate parabolic equations, Trans. Amer. Math. Soc. 271 (1982), 733-748.

Institute for Applied Mathematics, University of Hannover, 30167 Hannover, Germany e-mail: escher@ifam.uni-hannover.de

Fachbereich Mathematik und Informatik, Martin-Luther-Universität Halle-Wittenberg, 60120 Halle, Germany e-mail: anokd@mathematik.uni-halle.de

Department of Mathematics, Vanderbilt University, Nashville, TN 37240, USA

e-mail: simonett@math.vanderbilt.edu

Eingegangen 5. Oktober 1999 